\title{
On domain symmetry and its use in homogenization
}

\author{
Cristian Barbarosie ${ }^{1}$, Daniel A. Tortorelli ${ }^{* 2}$, and Seth Watts $^{2,3}$ \\ ${ }^{1}$ Department of Mathematics, University of Lisbon \\ ${ }^{2}$ Department of Mechancial Sciences and Engineering, University of Illinois at \\ Urbana-Champaign \\ ${ }^{3}$ Lawrence Livermore National Laboratory
}

August 1, 2016

\begin{abstract}
The present paper focuses on solving partial differential equations in domains exhibiting symmetries and periodic boundary conditions for the purpose of homogenization. We show in a systematic manner how the symmetry can be exploited to significantly reduce the complexity of the problem and the computational burden. This is especially relevant in inverse problems, when one needs to solve the partial differential equation (the primal problem) many times in an optimization algorithm. The main motivation of our study is inverse homogenization used to design architected composite materials with novel properties which are being fabricated at ever increasing rates thanks to recent advances in additive manufacturing. For example, one may optimize the morphology of a two-phase composite unit cell to achieve isotropic homogenized properties with maximal bulk modulus and minimal Poisson ratio. Typically, the isotropy is enforced by applying constraints to the optimization problem. However, in two dimensions, one can alternatively optimize the morphology of an equilateral triangle and then rotate and reflect the triangle to form a space filling $D_{3}$ symmetric hexagonal unit cell that necessarily exhibits isotropic homogenized properties. One can further use this $D_{3}$ symmetry to reduce the computational expense by performing the "unit strain" periodic boundary condition simulations on the single triangle symmetry sector rather than the six fold larger hexagon. In this paper we use group representation theory to derive the necessary periodic boundary conditions on the symmetry sectors of unit cells. The developments are done in a general setting, and specialized to the two-dimensional dihedral symmetries of the abelian $D_{2}$, i.e. orthotropic, square unit cell and nonabelian $D_{3}$, i.e. trigonal, hexagon unit cell. We then demonstrate how this theory can be applied by evaluating the homogenized properties of a two-phase planar composite over the triangle symmetry sector of a $D_{3}$ symmetric hexagonal unit cell.

Keywords : symmetric domain; finite element method; inverse homogenization
\end{abstract}

\section{Introduction}

Group representation theory has frequently been applied to reduce the cost of finite element and boundary element simulations of linear elastic structures that exhibit domain symmetry. However, the theory is seldom applied to reduce the cost of the "unit strain" simulations over morphological symmetric unit cells that are required to evaluate the unit cell's homogenized properties. This is despite the fact that the unit cells of many composite materials exhibit such morphological symmetries. In the following we revisit the group representation theory as it applies to the simulation of linear elastic structures that exhibit domain symmetry and extend the theory to address the periodic boundary condition computations that are required for homogenization.

The number of applications of group theory to boundary-value problems defined on domains (and their corresponding meshes) with symmetry are many. In the numerical approach, group theory is applied to the discretized governing partial differential equations and used to transform the stiffness matrix into one

*corresponding author, dtortore@illinois.edu

(C) 2016. This manuscript version is made available under the Elsevier user license

http://www.elsevier.com/open-access/userlicense/1.0/ 
with block diagonal form whereby the single large linear solve is replaced with a series of smaller linear solves. In effect, the solution space $\mathbb{R}^{n}$ is partitioned into a series of systematically derived complementary subspaces, as done in e.g. modal analysis methods. Refer to [1-4] for superb expositions of this approach. In the analytical approach the group theory is applied to the governing partial differential equations and used to partition the response space into complementary subspaces which enables a reduction of the problem domain $\Omega$ to the symmetry sector $C$. The means for defining the complementary subspaces and the boundary conditions/constraints over the new "cut" surfaces of $C$ are derived in a systematic manner. Excellent presentations of such approaches appear in [5-7].

Our main motivation comes from inverse homogenization where one seeks to design the microgeometry of a composite material in order to optimize certain macroscopic properties, see, e.g., [8]. Surprisingly, group theory has not played a significant role in the homogenization computations over unit cells, despite the fact that many unit cells exhibit numerous symmetries. The symmetric unit cell topic is addressed in [9, 10], however group theory is not applied and only simple orthotropic and reflective symmetries are considered.

In the following sections we show how to replace the single problem on the symmetric domain with smaller ones on the symmetry cell and how to exploit this for the purpose of homogenization. We first investigate domains characterized by abelian and later by nonabelian symmetry groups. In both cases we discuss scalar and vector valued elliptic partial differential equation problems as they relate to, e.g. linear heat conduction and linear elasticity. The general theory is presented, and the dihedral symmetries $D_{2}$ and $D_{3}$, i.e. bodies characterized by 2 and 3 rotational and reflective symmetries, are used to exemplify the abelian and nonabelian cases. We close our story by applying this theory to evaluate the homogenized properties of a two-phase planar composite over the triangle symmetry sector of a $D_{3}$ symmetric hexagonal unit cell.

\subsection{One-dimensional problems}

Before proceeding we discuss the analytical approach for the simple example of a uniaxially loaded linear elastic rod. First we note that for ordinary functions on the symmetric one-dimensional domain $\Omega=[-L, L]$, we speak of even $w_{e}: \Omega \rightarrow \mathcal{R}$ and odd $w_{o}: \Omega \rightarrow \mathcal{R}$ functions that satisfy $w_{e}(-x)=w_{e}(x)$ and $w_{o}(-x)=$ $-w_{o}(x)$. At any point $x \in \Omega$ we can decompose any function $w: \Omega \rightarrow \mathcal{R}$ into the sum of its even and odd components functions. Indeed, noting that $w(x)=1 / 2(w(x)+w(-x))+1 / 2(w(x)-w(-x))$ we define the even function $w_{e}: \Omega \rightarrow \mathcal{R}$ such that $w_{e}(x)=1 / 2(w(x)+w(-x))$ and the odd function $w_{o}: \Omega \rightarrow \mathcal{R}$ such that $w_{o}(x)=1 / 2(w(x)-w(-x))$ thereby giving us the decomposition $w(x)=w_{e}(x)+w_{o}(x)$. These even $w_{e}$ and odd $w_{o}$ functions are projections of the function $w: \Omega \rightarrow \mathcal{R}$ onto the even and odd subspaces $\mathcal{H}_{e}=\{w: \Omega \rightarrow \mathcal{R} \mid w(x)=w(-x)\}$ and $\mathcal{H}_{o}=\{w: \Omega \rightarrow \mathcal{R} \mid w(x)=-w(-x)\}$ in much the same way that the components $x_{i} \mathbf{e}_{i}$ of a vector $\mathbf{x}=\sum x_{i} \mathbf{e}_{i}$ are projections onto the basis vectors $\mathbf{e}_{i}$. In this way, we decompose $w$ into a linear combination of functions with restrictive properties, i.e. being even and odd, just as we decompose vectors into their restrictive components, i.e. being linear combinations of basis vectors. Moreover, we can construct the function $w$ on the entirety of $\Omega$ from the components $w_{e}$ and $w_{o}$ defined on solely the symmetry cell $C=[0, L]$. Indeed, for each $x \in C=[0, L]$ we have $w(x)=w_{e}(x)+w_{o}(x)$ and for each $x \in[-L, 0]$ we have $w(x)=w_{e}(-x)-w_{o}(-x)$. This decomposition has possible benefits if $w$ is the response of a differential equation wherein we can solve simpler problems for $w_{e}$ and $w_{o}$ over the smaller symmetry cell $C$ and reconstruct $w$ on the entirety of $\Omega$ from $w_{e}$ and $w_{o}$.

To further exemplify this decomposition we solve the ordinary differential equation which describes the elongation of an axially loaded rod in terms of the displacement $u$, Young's modulus $E$ and body load $b$ on the symmetric domain $\Omega=[-L, L]$, i.e.

$$
\begin{aligned}
\left(E(x) u^{\prime}(x)\right)^{\prime}+b(x) & =0 \quad \text { for } x \in \Omega=[-L, L] \\
u(L) & =0 \\
u(-L) & =0
\end{aligned}
$$

where the symmetric domain $\Omega$ also satisfies $E(-x)=E(x)$.

To demonstrate the analytical approach we solve the above problem for the choices $b(x)=5 x+2$ and $E(x)=10\left(x^{2}+2\right)$ (which satisfies the $E(-x)=E(x)$ symmetry condition). Using superposition and group theory we decompose $b$ into its even $b_{e}(x)=1 / 2(b(x)+b(-x))$ and odd $b_{o}(x)=1 / 2(b(x)-b(-x))$ 
components and solve two ordinary differential equations over the symmetry cell $C=[0, L]$ for the even displacement $u_{e}$

$$
\begin{array}{rll}
\left(E(x) u_{e}^{\prime}(x)\right)^{\prime}+b_{e}(x) & =0 & \text { for } x \in C=[0, L] \\
u_{e}(L) & =0 \\
E(0) u_{e}^{\prime}(0) & =0
\end{array}
$$

and the odd displacement $u_{o}$

$$
\begin{aligned}
\left(E(x) u_{o}^{\prime}(x)\right)^{\prime}+b_{o}(x) & =0 \quad \text { for } x \in C=[0, L] \\
u_{o}(L) & =0 \\
u_{o}(0) & =0 .
\end{aligned}
$$

Upon solving for $u_{e}$ and $u_{o}$ we have $u(x)=u_{e}(x)+u_{o}(x)$ for $x \in C=[0, L]$ and $u(x)=u_{e}(-x)-u_{o}(-x)$ for $x \in[-L, 0]$. $^{1}$

The boundary conditions at the $x=0$ cut follow from enforcing the zero jump conditions of displacement $u$ and stress $E u^{\prime}$, e.g. $\lim _{\epsilon \rightarrow 0^{+}}(u(\epsilon)-u(-\epsilon))=0$ and $\lim _{\epsilon \rightarrow 0^{+}}\left(E(\epsilon) u^{\prime}(\epsilon)-E(-\epsilon) u^{\prime}(-\epsilon)\right)=0$. The first limit is trivially satisfied for even functions since $u_{e}(\epsilon)-u_{e}(-\epsilon)=u_{e}(\epsilon)-u_{e}(\epsilon)=0$, however the second requires $E(\epsilon) u_{e}^{\prime}(\epsilon)=0$ since $E(\epsilon) u_{e}^{\prime}(\epsilon)-E(-\epsilon) u_{e}^{\prime}(-\epsilon)=E(\epsilon) u_{e}^{\prime}(\epsilon)+E(\epsilon) u_{e}^{\prime}(\epsilon)=2 E(\epsilon) u_{e}^{\prime}(\epsilon)$. Indeed, these results follow from the equalities $E(-x)=E(x)$ and $u_{e}(-x)=u_{e}(x)$, the latter which implies $u_{e}^{\prime}(-x)=-u_{e}^{\prime}(x)$. A similar derivation is used to determine the odd function boundary condition at the $x=0$ cut. The rational for solving the two problems for $u_{e}$ and $u_{o}$ on the smaller symmetry cell $C$ rather than one problem for $u$ on the larger domain $\Omega$ is that it may be more efficient, especially if finite element simulations are used, to solve two smaller problems rather than one larger problem. Note that the analytical approach requires the application of new boundary conditions at the $x=0$ cut.

To get a feel of what is involved in homogenization theory, we again resort to our simple uniaxial rod, which we now view as the symmetric unit cell $\Omega=[-1,1]$. First we solve the "unit strain" problem subject to periodic boundary conditions, i.e. we evaluate the response $u$ such that

$$
\begin{aligned}
\left(E(x)\left(u^{\prime}(x)-1\right)\right)^{\prime} & =0 \quad \text { for } x \in \Omega=[-1,1] \\
u(-1) & =u(1) \\
u(0) & =0
\end{aligned}
$$

where the $u(0)=0$ condition is enforced to prevent rigid body modes.

Akin to the decomposition of $b$, we decompose the unit strain into its even and odd components. Using the equalities $u_{e}^{\prime}(-x)=-u_{e}^{\prime}(x)$ and $u_{o}^{\prime}(-x)=u_{o}^{\prime}(x)$ we see that the unit strain is an odd function since the unit strain equals 1 at both $x$ and $-x$. Thusly we need only solve the odd problem on the symmetry cell

$$
\begin{array}{rlr}
\left(E(x)\left(u_{o}^{\prime}(x)-1\right)\right)^{\prime} & =0 & \\
u_{o}(1) & =0 & \\
u_{o}(0) & =0 &
\end{array}
$$

The $u_{o}(1)=0$ condition follows from the periodicity $u_{o}(-1)=u_{o}(1)$ and odd function $u_{o}(-1)=-u_{o}(1)$ equalities. As seen above, we replaced the unit cell problem for $u$ on the larger domain $\Omega$ with that for $u_{o}$ on the smaller symmetry cell $C$. Having the periodic response we evaluate the homogenized modulus over

\footnotetext{
$u(x)$.

${ }^{1}$ The assertion that $u=u_{e}+u_{o}$ over $C$ follows from substitution, i.e. $u=u_{e}+u_{o}=1 / 2(u(x)+u(-x))+1 / 2(u(x)-u(-x))=$
} 


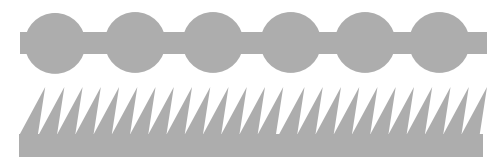

(a) Invariant to translations.

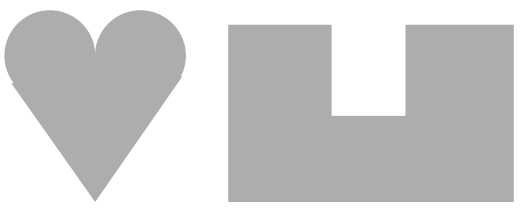

(b) Invariant to a reflection.

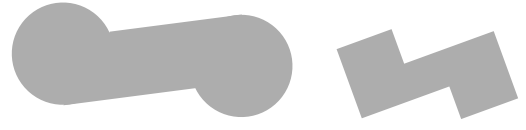

(c) Invariant to a rotation.

Figure 1: Plane region symmetry illustrations.

the smaller symmetry sector $C$. Indeed

$$
\begin{aligned}
E^{h} & =\frac{1}{2} \int_{-1}^{1} E(x)\left(1+u^{\prime}(x)\right) d x \\
& =\frac{1}{2} \int_{-1}^{0} E(x)\left(1+u_{o}^{\prime}(-x)\right) d x+\frac{1}{2} \int_{0}^{1} E(x)\left(1+u_{o}^{\prime}(x)\right) d x \\
& =\frac{1}{2} \int_{1}^{0} E(g(y))\left(1+u_{o}^{\prime}(-g(y))\right) g^{\prime}(y) d y+\frac{1}{2} \int_{0}^{1} E(x)\left(1+u_{o}^{\prime}(x)\right) d x \\
& =\frac{1}{2} \int_{0}^{1} E(y)\left(1+u_{o}^{\prime}(y)\right) d y+\frac{1}{2} \int_{0}^{1} E(x)\left(1+u_{o}^{\prime}(x)\right) d x \\
& =\int_{0}^{1} E(x)\left(1+u_{o}^{\prime}(x)\right) d x,
\end{aligned}
$$

where we used the change of variable theorem with $x=g(y)=-y$ and the equalities $E(-x)=E(x)$ and $u_{o}^{\prime}(-x)=u_{o}^{\prime}(x)$.

\subsection{Isometries of the plane, invariant sets and functions}

In two dimensions, the notion of symmetry is obviously more complex than the above one-dimensional examples, and it is better described as invariance with respect to a family of isometries of the plane. A mapping $\sigma: \mathcal{R}^{2} \rightarrow \mathcal{R}^{2}$ is called an isometry if it preserves the distance between points, that is, $\operatorname{dist}\left(\sigma\left(\mathbf{x}_{1}\right), \sigma\left(\mathbf{x}_{2}\right)\right)=\operatorname{dist}\left(\mathbf{x}_{1}, \mathbf{x}_{2}\right)$, for all $\mathbf{x}_{1}, \mathbf{x}_{2} \in \mathcal{R}^{2}$.

The above definition is a particular case of the more general notion of isometry for higher dimensional spaces $\mathcal{R}^{n}$, or, even more generally, for arbitrary metric spaces. These cases are outside the scope of the present work. Isometries of the plane $\mathcal{R}^{2}$ are : translations, rotations around a fixed point and reflections with respect to a fixed straight line. Note that translations and rotations preserve orientation, while reflections do not.

A point $\mathbf{x} \in \mathcal{R}^{2}$ is called a "fixed point" with respect to a certain isometry $\sigma$ if $\sigma(\mathbf{x})=\mathbf{x}$. Translations have no fixed points. Rotations have exactly one fixed point (the center of rotation). Reflections have many fixed points (any point belonging to the axis of reflection). It can be proven that, if the origin $(0,0)$ is a fixed point for a certain isometry $\sigma$, then $\sigma$ is a linear transformation. Thus, $\sigma$ can be represented by a matrix : $\sigma(\mathbf{x})=\mathbf{R} \mathbf{x}$. The matrix $\mathbf{R}$ is orthogonal, that is, $\mathbf{R}^{-1}=\mathbf{R}^{T}$.

A subset $A$ of $\mathcal{R}^{2}$ is said to be invariant to a certain isometry $\sigma$ if $\sigma(A)=A$; in other words, $\mathbf{x} \in A$ if and only if $\sigma(\mathbf{x}) \in A$.

Subsets invariant with respect to translations are not very interesting for the purpose of this work, since they are unbounded. Two examples are shown in Figure 1a. Figure 1b shows two examples of sets which are invariant to a reflection, while Figure 1c exhibits two sets invariant to a $\pi$ rotation.

Of course there are "more symmetric" domains, which are invariant to several isometries, as we shall see in the sequel.

A function $f: \mathcal{R}^{2} \rightarrow \mathcal{R}$ is said to be invariant to composition with a certain isometry $\sigma$ if $f(\sigma(\mathbf{x}))=f(\mathbf{x})$ for all $\mathbf{x} \in \mathcal{R}^{2}$. Note that this can be written equivalently as $f\left(\sigma^{-1}(\mathbf{x})\right)=f(\mathbf{x})$ for all $\mathbf{x}$. Or, in short notation, $f \circ \sigma=f \circ \sigma^{-1}=f$. For instance, functions $x+y$ and $x y$ are invariant to the reflection $\sigma(x, y)=(y, x)$. The function $x^{2}+y^{2}$ is invariant to any rotation around the origin, and also to reflections with respect to 
any straight line passing through the origin. Constant functions are invariant to everything. The function $f$ does not have to be defined in the whole $\mathcal{R}^{2}$. Instead, $f$ may be defined in a region $\Omega \subset \mathcal{R}^{2}$, provided $\Omega$ itself is invariant.

Until now we have discussed invariance to one isometry $\sigma$. Now we shall focus on several isometries at once, that is, we shall consider a family of isometries which we may generically denote by $\mathcal{G}$. If a domain $\Omega$ (or a function $f$ ) is invariant to two isometries $\sigma$ and $\eta$, then it is also invariant to their compositions $\sigma \circ \eta$ and $\eta \circ \sigma$. Another, related, property is that invariance with respect to an isometry $\sigma$ implies invariance with respect to its inverse $\sigma^{-1}$. Due to the above properties, it is natural to consider a family $\mathcal{G}$ of isometries which is closed to composition and to the operation of inverse. As a consequence the identity is necessarily an element of the family. Such a family forms an algebraic structure called group. In this work we shall focus on finite groups. Isometries in the group will be denoted by $\sigma_{g}$ and their matrix representations will be denoted by $\mathbf{R}_{g}$.

Consider now a group $\mathcal{G}$ of isometries of the plane and an arbitrary function $f: \mathcal{R}^{2} \rightarrow \mathcal{R}$ (not necessarily invariant). One can "extract" from $f$ the part which is invariant to $\mathcal{G}$ by introducing

$$
\tilde{f}(\mathbf{x})=\frac{1}{n} \sum_{g \in \mathcal{G}} f\left(\mathbf{R}_{g}^{-1} \mathbf{x}\right)
$$

In the above summation, $n$ is the number of elements of $\mathcal{G}$; therefore this is the same as $\tilde{f}(\mathbf{x})=\frac{1}{n} \sum_{g \in \mathcal{G}} f\left(\mathbf{R}_{g} \mathbf{x}\right)$. It is a simple exercise to check that $\tilde{f}$ is indeed invariant to $\mathcal{G}$. Note also that, if $f$ is already invariant to $\mathcal{G}$, then $\tilde{f}=f$; this is why we may call $\tilde{f}$ "a projection" of $f$ on the space of invariant functions and introduce the notation $\tilde{f}=P_{1} f$.

If we turn our attention on what "remains" from the function $f$ after extracting its invariant part, that is, on $f-P_{1} f$, things become more complicated. One possibility is simply to say that $f-P_{1} f$ is just another projection of $f$. While this is certainly true, it is an interesting question to describe the space onto which we are projecting, that is, the space of functions $\bar{f}$ such that $\bar{f}=\bar{f}-P_{1} \bar{f}$, or $P_{1} \bar{f}=0$. Another interesting question is to try to further decompose this space into smaller spaces of "specially behaved" functions.

A classical example is the group containing only two elements : the identity $\mathbf{R}_{1}$ and the reflection with respect to the vertical axis, denoted by $\mathbf{R}_{y}$ and defined by $\mathbf{R}_{y}(x, y)=(-x, y)$; note that $\mathbf{R}_{y}$ is involutory, i.e. $\mathbf{R}_{y}^{-1}=\mathbf{R}_{y}$. This group is called $D_{1}$; domains invariant to this group are represented in Figure $1 \mathrm{~b}$. For an arbitrary funtion $f$, the invariant part $\tilde{f}=P_{1} f$ is even in the $x$ variable, while the "remainder" $\bar{f}=f-P_{1} f$ is odd in the same variable. This example is presented in the above one-dimension discussion. For the group $D_{1}$, the two spaces onto which we are projecting are clear : $P_{1}$ projects onto the space of even functions, while the "remainder" projects onto the space of odd functions (in the $x$ variable).

Another simple example is the group $C_{2}$, containing the identity $\mathbf{R}_{1}$ and the involutory $\pi$ rotation which we shall denote by $\mathbf{R}_{x y}$. Domains invariant to this group are represented in Figure 1c. Again, $P_{1}$ projects onto the space of even functions, this time in both variables $(x, y)$, while the "remainder" is odd in both variables.

The next step towards more complicated groups is perhaps $C_{3}$, consisting of three elements : the identity, the $2 \pi / 3$ rotation $\mathbf{R}_{2}$ and (of course) the $4 \pi / 3$ rotation $\mathbf{R}_{4}$. For this group, we have a fairly good idea of the space onto which $P_{1}$ projects; it contains all functions which are left unchanged by the $2 \pi / 3$ rotation (invariance to the $4 \pi / 3$ rotation follows as a consequence). But the "remainder" belongs to a rather foggy space. It is the space of all functions $f$ such that

$$
f(\mathbf{x})+f\left(\mathbf{R}_{2}(\mathbf{x})\right)+f\left(\mathbf{R}_{4}(\mathbf{x})\right)=0 .
$$

They are vaguely similar to an odd function. One might expect to further decompose this space in a sum of two narrower function spaces. As it turns out, this is not possible if we stick to real-valued functions. But if we allow for complex-valued functions, then the space of functions $f$ satisfying Equation 1 can be further decomposed as follows. Denote by $\xi$ the complex cubic root of 1 , that is, $\xi=e^{2 \pi i / 3}$. One may consider the space of functions with the property

$$
f\left(\mathbf{R}_{2}(\mathbf{x})\right)=\xi f(\mathbf{x}) \text { for all } \mathbf{x}
$$

and, as a consequence, $f\left(\mathbf{R}_{4}(\mathbf{x})\right)=\xi^{2} f(\mathbf{x})$. And there are functions $f$ such that

$$
f\left(\mathbf{R}_{2}(\mathbf{x})\right)=\xi^{2} f(\mathbf{x}) \text { for all } \mathbf{x}
$$


and, as a consequence, $f\left(\mathbf{R}_{4}(\mathbf{x})\right)=\xi^{4} f(\mathbf{x})=\xi f(\mathbf{x})$. It can be proven that any function $f$ satisfying Equation 1 can be written as a sum between a function satisfying Equation 2 and another one satisfying 3 . In other words, an arbitrary complex-valued function $f$ can be written as the sum of three parts, one satisfying Equation 1, another one satisfying 2 and a third one satisfying 3 . These three components of $f$ can be explicitly constructed as

$$
\begin{aligned}
P_{1} f(\mathbf{x}) & =\frac{1}{3}\left(f(\mathbf{x})+f\left(\mathbf{R}_{2}^{-1} \mathbf{x}\right)+f\left(\mathbf{R}_{4}^{-1}(\mathbf{x})\right)\right) \\
P_{2} f(\mathbf{x}) & =\frac{1}{3}\left(f(\mathbf{x})+\xi f\left(\mathbf{R}_{2}^{-1}(\mathbf{x})\right)+\xi^{2} f\left(\mathbf{R}_{4}^{-1}(\mathbf{x})\right)\right. \\
P_{3} f(\mathbf{x}) & =\frac{1}{3}\left(f(\mathbf{x})+\xi^{2} f\left(\mathbf{R}_{2}^{-1}(\mathbf{x})\right)+\xi^{4} f\left(\mathbf{R}_{4}^{-1}(\mathbf{x})\right)=\frac{1}{3}\left(f(\mathbf{x})+\xi^{2} f\left(\mathbf{R}_{2}^{-1}(\mathbf{x})\right)+\xi f\left(\mathbf{R}_{4}^{-1}(\mathbf{x})\right)\right.\right.
\end{aligned}
$$

We thus obtain three complex projector operators, $P_{1}, P_{2}$ and $P_{3}$. Since $1+\xi+\xi^{2}=0$, it is easy to see that $P_{1} f+P_{2} f+P_{3} f=f$.

The above procedure can be partially mimicked for real-valued functions, roughly by considering the real part and the imaginary part of the complex projections of a real-valued function. This gives rise to operators $P_{2}^{1}$ and $P_{2}^{2}$ given by

$$
\begin{gathered}
P_{2}^{1} f(\mathbf{x})=\frac{2}{3} f(\mathbf{x})-\frac{1}{3} f\left(\mathbf{R}_{2}^{-1}(\mathbf{x})\right)-\frac{1}{3} f\left(\mathbf{R}_{4}^{-1}(\mathbf{x})\right) \\
P_{2}^{2} f(\mathbf{x})=\frac{\sqrt{3}}{3} f\left(\mathbf{R}_{2}^{-1}(\mathbf{x})\right)-\frac{\sqrt{3}}{3} f\left(\mathbf{R}_{4}^{-1}(\mathbf{x})\right)
\end{gathered}
$$

It is easy to check that, for an arbitrary function $f$, one has $f=P_{1} f+P_{2}^{1} f$. Oddly enough, $P_{2}^{2}$ does not contribute to this decomposition (roughly speaking, this happens because $P_{2}^{2}$ comes from the imaginary part of the complex projectors, while we are dealing with a real-valued function $f$ ). The operators $P_{1}$ and $P_{2}^{1}$ are idempotent, that is, $P_{1} P_{1} f=P_{1} f, P_{2}^{1} P_{2}^{1} f=P_{2}^{1} f$ and orthogonal, that is $P_{1} P_{2}^{1} f=P_{2}^{1} P_{1} f=0$, but $P_{2}^{2}$ is not idempotent : $P_{2}^{2} P_{2}^{2} f=-P_{2}^{1} f$. Thus, $P_{2}^{2}$ is not a true projector.

However, $P_{2}^{2}$ plays an important role when one seeks to describe the behaviour of an arbitrary function $f$ upon composition with one of the isometries in $C_{3}$, e.g. with $\mathbf{R}_{2}$. Direct computations show that

$$
f\left(\mathbf{R}_{2} \mathbf{x}\right)=P_{1} f(\mathbf{x})-\frac{1}{2} P_{2}^{1} f(\mathbf{x})+\frac{\sqrt{3}}{2} P_{2}^{2} f(\mathbf{x})
$$

Thus, the operator $P_{2}^{2}$ is more like a shadow of $P_{2}^{1}$ which appears in the light of the action of the group $C_{3}$ on a function $f$.

The above considerations will be discussed in more detail and in a more general setting, using the notion of irreducible representation, or irreps. Abelian groups will be discussed, with special emphasis on $D_{2}$, as well as nonabelian groups, with special emphasis on $D_{3}$.

\section{Scalar valued elliptic problems}

We are concerned with solving an elliptic boundary value problem of finding the scalar response $w \in \mathcal{H}\left(\Omega, w^{p}\right)$ such that

$$
\int_{\Omega} \nabla v \cdot \mathbf{k} \nabla w d v=\int_{\Omega}(\nabla v \cdot \mathbf{k} \chi+v f) d v+\int_{\partial \Omega^{n}} v q^{p} d a
$$

for all $v \in \mathcal{H}(\Omega, 0)$ where

$$
\mathcal{H}(\Omega, a)=\left\{z: \Omega \rightarrow \mathcal{R} \mid z \text { and } \nabla z \text { are square integrable over } \Omega \text { and } z=a \text { on } \partial \Omega^{d}\right\}
$$

In the above, $\Omega$ represents the region in space occupied by the body whose material is described by the constitutive tensor $\mathbf{k}$ and subjected to the source $f$ and initial flux $-\operatorname{div}(\mathbf{k} \boldsymbol{\chi})$ loads where the vector $\chi$ is analogous to an initial strain. The boundary of $\Omega$ is partitioned into the complementary surfaces $\partial \Omega^{d}$ and $\partial \Omega^{n}$ over which the response $w^{p}$ and normal flux $q^{p}=\mathbf{k}(\nabla w-\boldsymbol{\chi}) \cdot \mathbf{n}$ where $\mathbf{n}$ is the surface normal vector, are prescribed. This problem appears in, e.g. heat conduction where $w$ is the temperature field. 
We are interested in bodies that have domain symmetry with respect to a finite set $\mathcal{G}=\left\{\mathbf{R}_{1}, \mathbf{R}_{2}, \cdots\right\}$ of orthogonal transformations $\mathbf{R}_{i}$ meaning that for each $\mathbf{x} \in \Omega$ we have $\mathbf{R}_{i} \mathbf{x} \in \Omega$ and similarly for any $\mathbf{x} \in \partial \Omega^{n}$ we have $\mathbf{R}_{i} \mathbf{x} \in \partial \Omega^{n}$ and likewise for any $\mathbf{x} \in \partial \Omega^{d}$ we have $\mathbf{R}_{i} \mathbf{x} \in \partial \Omega^{d}$, cf. Figure 2. Finally, the material constitutive tensor $\mathbf{k}$ at $\mathbf{x}$ and $\mathbf{R}_{i} \mathbf{x}$ satisfies $\mathbf{k}(\mathbf{x})=\mathbf{R}_{i}^{-1} \mathbf{k}\left(\mathbf{R}_{i} \mathbf{x}\right) \mathbf{R}_{i}$. As mentioned above, the set $\mathcal{G}$ of these symmetry transformations is closed meaning that $\mathbf{R}_{i} \mathbf{R}_{j}$ is in the set as well as $\mathbf{R}_{i}^{-1}$, i.e. $\mathbf{R}_{i} \mathbf{R}_{j} \in \mathcal{G}$ and $\mathbf{R}_{i}^{-1} \in \mathcal{G}$ and of course then we have the identity $\mathbf{I} \in \mathcal{G}$.
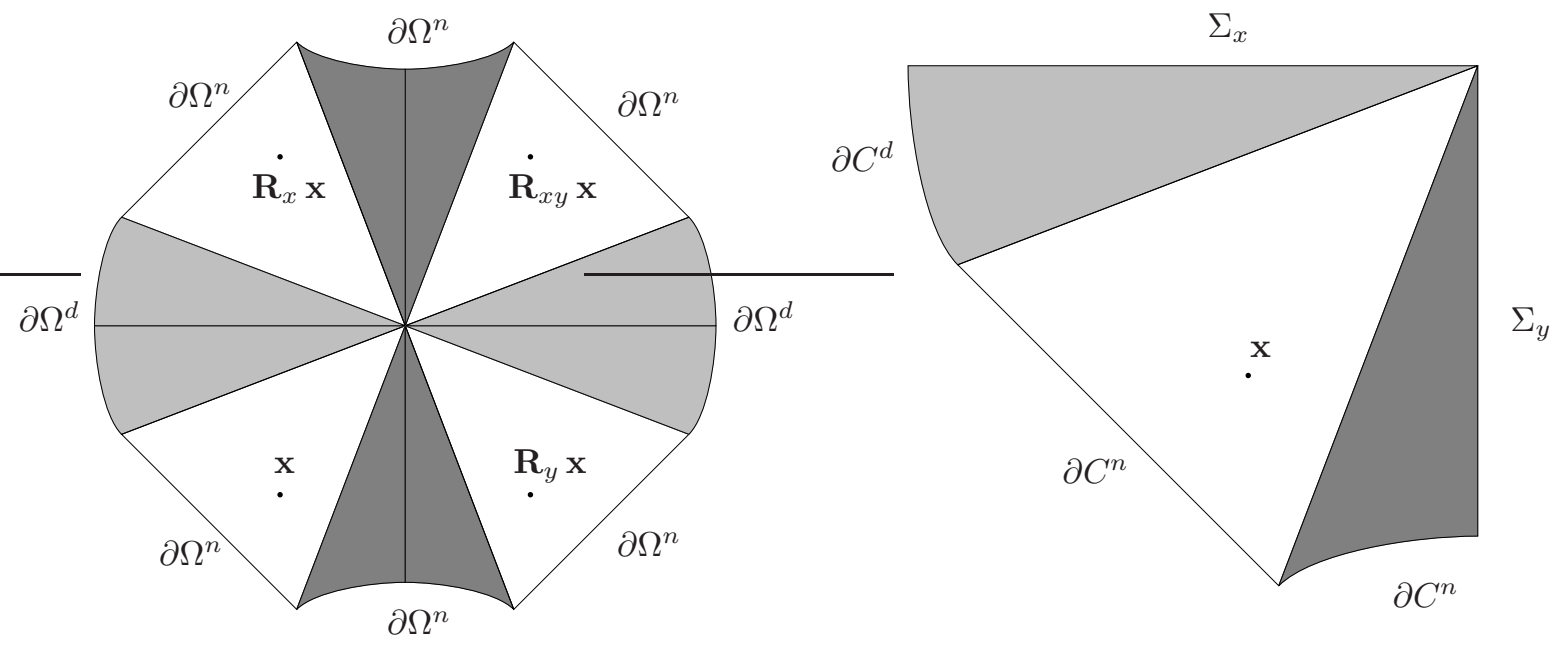

Figure 2: $D_{2}$ symmetric domain $\Omega$ (left) and symmetry cell $C$ (right).

\subsection{Abelian symmetry}

To put our abelian study in context we consider a plane domain with two orthogonal axes of reflective symmetry, i.e. $D_{2}$, we have order $n=4$ elements of the symmetry group $\mathcal{G}=\left\{\sigma_{1}, \sigma_{y}, \sigma_{x}, \sigma_{x y}\right\}$. These group elements $\sigma_{g}$ are tabulated below along with their transformations $\mathbf{R}_{g}$ (given with respect to the usual orthonormal coordinate system with basis vectors $\left\{\mathbf{e}_{1}, \mathbf{e}_{2}\right\}$ )

\begin{tabular}{|c|c|c|c|c|c|}
\hline$\sigma_{1}$ & \multicolumn{2}{|c|}{$\sigma_{x}$} & $\sigma_{y}$ & $\sigma_{x y}$ \\
\hline \hline $\mathbf{R}_{1}=\left[\begin{array}{ll}1 & 0 \\
0 & 1\end{array}\right]$ & $\mathbf{R}_{x}=\left[\begin{array}{cc}1 & 0 \\
0 & -1\end{array}\right]$ & $\mathbf{R}_{y}=\left[\begin{array}{cc}-1 & 0 \\
0 & 1\end{array}\right]$ & $\mathbf{R}_{x y}=\left[\begin{array}{cc}-1 & 0 \\
0 & -1\end{array}\right]$ \\
\hline
\end{tabular}

which respectively correspond to the identity $\sigma_{1}$, reflection about the $x$ axis $\sigma_{x}$, reflection about the $y$ axis $\sigma_{y}$, and reflection about the $x$ and then $y$ axes $\sigma_{x y}$, or equivalently a $2 \pi$ rotation about the $z$-axis $\sigma_{1}$, reflection about the $x$ axis $\sigma_{x}$, a reflection about the $y$ axis $\sigma_{y}$, and a $\pi$ rotation about the $z$-axis $\sigma_{x y}$ and hence we have the 2 rotational and 2 reflective symmetries. As always, the transformations are orthogonal, i.e. $\mathbf{R}_{g}^{-1}=\mathbf{R}_{g}^{T}$. (And for this special case the transformations are involutory, i.e. $\mathbf{R}_{g}^{-1}=\mathbf{R}_{g}$.) Thusly we see that for each element $\sigma_{g} \in \mathcal{G}$ we have $\sigma_{g}^{-1} \in \mathcal{G}$.

We verify that the composition of any two elements in $\mathcal{G}$ is also in $\mathcal{G}$, e.g. $\sigma_{y} \sigma_{x}=\sigma_{x y} \in \mathcal{G}$ through the $D_{2}$ group multiplication, i.e. Cayley, table

\begin{tabular}{|c||c|c|c|c|}
\hline & $\sigma_{1}$ & $\sigma_{x}$ & $\sigma_{y}$ & $\sigma_{x y}$ \\
\hline \hline$\sigma_{1}$ & $\sigma_{1}$ & $\sigma_{x}$ & $\sigma_{y}$ & $\sigma_{x y}$ \\
\hline$\sigma_{x}$ & $\sigma_{x}$ & $\sigma_{1}$ & $\sigma_{x y}$ & $\sigma_{y}$ \\
\hline$\sigma_{y}$ & $\sigma_{y}$ & $\sigma_{x y}$ & $\sigma_{1}$ & $\sigma_{x}$ \\
\hline$\sigma_{x y}$ & $\sigma_{x y}$ & $\sigma_{y}$ & $\sigma_{x}$ & $\sigma_{1}$ \\
\hline
\end{tabular}

In reading this table we compose the column element with the row element, so that e.g. for row 3 and column 2 we have $\sigma_{y} \sigma_{x}=\sigma_{x y}$. The important thing to note is that each element of the group appears once in each 
row of the table. For this abelian group the row/column order is inconsequential since the composition operation is commutative, e.g. $\sigma_{x} \sigma_{y}=\sigma_{y} \sigma_{x}=\sigma_{x y}$.

The table of weighting coefficients, i.e. irreducible representations or irreps, for $D_{2}$, is given by

\begin{tabular}{|c||r|r|r|r|}
\hline$\rho_{i} \sigma_{g}$ & $\sigma_{1}$ & $\sigma_{x}$ & $\sigma_{y}$ & $\sigma_{x y}$ \\
\hline \hline$\rho_{1}$ & 1 & 1 & 1 & 1 \\
\hline$\rho_{2}$ & 1 & 1 & -1 & -1 \\
\hline$\rho_{3}$ & 1 & -1 & 1 & -1 \\
\hline$\rho_{4}$ & 1 & -1 & -1 & 1 \\
\hline
\end{tabular}

Some important properties to note are that 1) $\rho_{i}\left(\sigma_{1}\right)=1$ and $\left.\rho_{1}\left(\sigma_{g}\right)=1,2\right)$ the representations are orthogonal, i.e. $\rho_{i}\left(\sigma_{j}^{-1}\right)=\left[\rho_{i}\left(\sigma_{j}\right)\right]^{T}=\rho_{i}\left(\sigma_{j}\right)$ (this is more enlightening in the nonabelian case where the irreps are matrix valued), 3) the rows of the table are mutually orthogonal, 4) the magnitude of the row squared equals $n$, e.g. for row 2 we see that $1^{2}+(-1)^{2}+1^{2}+(-1)^{2}=4$ and 5$)$ the products of the irreps follow those of the group multiplication table, e.g. $\rho_{3}\left(\sigma_{y} \sigma_{x y}\right)=\rho_{3}\left(\sigma_{x}\right)=\rho_{3}\left(\sigma_{y}\right) \rho_{3}\left(\sigma_{x y}\right)$. Generalizing this last result yields

$$
\rho_{i}\left(\sigma_{j} \sigma_{k}\right)=\rho_{i}\left(\sigma_{j}\right) \rho_{i}\left(\sigma_{k}\right)
$$

It is also noted, without proof, that this table is unique. These properties are in fact used to derive the tables for our $D_{2}$ and $D_{3}$ studies as well as many other symmetry groups as discussed in [11].

A useful identity, derived from the irrep table, is

$$
\sum_{g \in \mathcal{G}} f\left(\rho_{i}\left(\sigma_{g}\right)\right)=\sum_{g \in \mathcal{G}} f\left(\rho_{i}\left(\sigma_{g}\right) \rho_{i}\left(\sigma_{h}\right)\right)=\sum_{g \in \mathcal{G}} f\left(\rho_{i}\left(\sigma_{g} \sigma_{h}\right)\right)
$$

for any function $f$. Indeed, the set of elements $\rho_{i}\left(\sigma_{g}\right)$ and $\rho_{i}\left(\sigma_{g} \sigma_{h}\right)=\rho_{i}\left(\sigma_{g}\right) \rho_{i}\left(\sigma_{h}\right)$ are the same due to the group closure property of the irreps.

From the $D_{2}$ table of irreps we construct four projectors defined such that for any scalar function $w \in$ $\mathcal{H}(\Omega)=\{z: \Omega \rightarrow \mathcal{R} \mid z$ and $\nabla z$ are square integrable over $\Omega\}$ we have

$$
\begin{aligned}
& P_{1} w(\mathbf{x})=\frac{1}{4}\left(w(\mathbf{x})+w\left(\mathbf{R}_{x}^{-1} \mathbf{x}\right)+w\left(\mathbf{R}_{y}^{-1} \mathbf{x}\right)+w\left(\mathbf{R}_{x y}^{-1} \mathbf{x}\right)\right) \\
& P_{2} w(\mathbf{x})=\frac{1}{4}\left(w(\mathbf{x})+w\left(\mathbf{R}_{x}^{-1} \mathbf{x}\right)-w\left(\mathbf{R}_{y}^{-1} \mathbf{x}\right)-w\left(\mathbf{R}_{x y}^{-1} \mathbf{x}\right)\right) \\
& P_{3} w(\mathbf{x})=\frac{1}{4}\left(w(\mathbf{x})-w\left(\mathbf{R}_{x}^{-1} \mathbf{x}\right)+w\left(\mathbf{R}_{y}^{-1} \mathbf{x}\right)-w\left(\mathbf{R}_{x y}^{-1} \mathbf{x}\right)\right) \\
& P_{4} w(\mathbf{x})=\frac{1}{4}\left(w(\mathbf{x})-w\left(\mathbf{R}_{x}^{-1} \mathbf{x}\right)-w\left(\mathbf{R}_{y}^{-1} \mathbf{x}\right)+w\left(\mathbf{R}_{x y}^{-1} \mathbf{x}\right)\right)
\end{aligned}
$$

The \pm signs, i.e. weights, come from the rows of the above irrep table. Generalizing the above (for abelian groups) gives

$$
P_{i} w(\mathbf{x})=\frac{1}{n} \sum_{g \in \mathcal{G}} \rho_{i}\left(\sigma_{g}\right) w\left(\mathbf{R}_{g}^{-1} \mathbf{x}\right)
$$

where the sum is over the group elements, e.g. $\sigma_{1}, \sigma_{x}, \sigma_{y}$ and $\sigma_{x y}$ for $D_{2}$. 
From the above we also see that

$$
\begin{aligned}
P_{i} w\left(\mathbf{R}_{h}^{-1} \mathbf{x}\right) & =\frac{1}{n} \sum_{g \in \mathcal{G}} \rho_{i}\left(\sigma_{g}\right) w\left(\mathbf{R}_{g}^{-1}\left(\mathbf{R}_{h}^{-1} \mathbf{x}\right)\right) \\
& =\frac{1}{n} \sum_{g \in \mathcal{G}} \overbrace{\rho_{i}\left(\sigma_{h}^{-1} \sigma_{h}\right)}^{=1} \rho_{i}\left(\sigma_{g}\right) w\left(\left(\mathbf{R}_{h} \mathbf{R}_{g}\right)^{-1} \mathbf{x}\right) \\
& =\frac{1}{n} \sum_{g \in \mathcal{G}} \rho_{i}\left(\sigma_{h}^{-1}\right) \rho_{i}\left(\sigma_{h}\right) \rho_{i}\left(\sigma_{g}\right) w\left(\left(\mathbf{R}_{h} \mathbf{R}_{g}\right)^{-1} \mathbf{x}\right) \\
& =\frac{1}{n} \sum_{g \in \mathcal{G}}\left[\rho_{i}\left(\sigma_{h}\right)\right]^{T} \rho_{i}\left(\sigma_{h} \sigma_{g}\right) w\left(\left(\mathbf{R}_{h} \mathbf{R}_{g}\right)^{-1} \mathbf{x}\right) \\
& =\rho_{i}\left(\sigma_{h}\right) \frac{1}{n} \sum_{g \in \mathcal{G}} \rho_{i}\left(\sigma_{h} \sigma_{g}\right) w\left(\left(\mathbf{R}_{h} \mathbf{R}_{g}\right)^{-1} \mathbf{x}\right) \\
& =\rho_{i}\left(\sigma_{h}\right) P_{i} w(\mathbf{x})
\end{aligned}
$$

Using the orthogonality of the irreps and manipulating the above also gives

$$
\begin{aligned}
P_{i} w\left(\mathbf{R}_{h} \mathbf{x}\right) & =\rho_{i}\left(\sigma_{h}^{-1}\right) P_{i} w(\mathbf{x}) \\
& =\left[\rho_{i}\left(\sigma_{h}\right)\right]^{T} P_{i} w(\mathbf{x}) \\
& =\rho_{i}\left(\sigma_{h}\right) P_{i} w(\mathbf{x})
\end{aligned}
$$

Upon combining Equations 5 and 6 we find

$$
\begin{aligned}
P_{j} P_{i} w(\mathbf{x}) & =\frac{1}{n} \sum_{g \in \mathcal{G}} \rho_{j}\left(\sigma_{g}\right) P_{i} w\left(\mathbf{R}_{g}^{-1} \mathbf{x}\right) \\
& =\frac{1}{n} \sum_{g \in \mathcal{G}} \rho_{j}\left(\sigma_{g}\right) \rho_{i}\left(\sigma_{g}\right) P_{i} w(\mathbf{x}) \\
& =\frac{1}{n} P_{i} w(\mathbf{x}) \sum_{g \in \mathcal{G}} \rho_{j}\left(\sigma_{g}\right) \rho_{i}\left(\sigma_{g}\right) \\
& =\frac{1}{n} P_{i} w(\mathbf{x}) n \delta_{i j} \\
& =\delta_{i j} P_{i} w(\mathbf{x})
\end{aligned}
$$

where we use the row-wise orthogonality of the irreps. The above implies that 1) $P_{i} P_{i} w(\mathbf{x})=P_{i} w(\mathbf{x})$ and 2) $P_{j} P_{i} w(\mathbf{x})=0$ for $i \neq j$, i.e. that the $P_{i}$ (acting on the arbitrary $w \in \mathcal{H}(\Omega)$ ) are orthogonal projections and the $P_{i} w$ live in the complementary spaces $P_{i} \mathcal{H}(\Omega)=\left\{z \in \mathcal{H}(\Omega) \mid P_{i} z=z\right\}$.

We next prove the result

$$
w(\mathbf{x})=\sum_{i=1}^{n} P_{i} w(\mathbf{x})
$$

Indeed,

$$
\begin{aligned}
\sum_{i=1}^{n} P_{i} w(\mathbf{x}) & =\sum_{i=1}^{n} \frac{1}{n} \sum_{g} \rho_{i}\left(\sigma_{g}\right) w\left(\mathbf{R}_{g}^{-1} \mathbf{x}\right) \\
& =\sum_{g} w\left(\mathbf{R}_{g}^{-1} \mathbf{x}\right) \sum_{i=1}^{n} \overbrace{\rho_{i}\left(\sigma_{1}\right)}^{=1} \frac{1}{n} \rho_{i}\left(\sigma_{g}\right) \\
& =\sum_{g} w\left(\mathbf{R}_{g}^{-1} \mathbf{x}\right) \delta_{1 g} \\
& =w\left(\mathbf{R}_{1}^{-1} \mathbf{x}\right) \\
& =w(\mathbf{x})
\end{aligned}
$$


where the $\sum_{i=1}^{n} \rho_{i}\left(\sigma_{1}\right) \frac{1}{n} \rho_{i}\left(\sigma_{g}\right)=\delta_{1 g}$ result follows from properties 3 and 4 of the irrep table. To see this we define the matrix $\boldsymbol{\rho}$ from the irrep table and use properties 3 and 4 to obtain $\boldsymbol{\rho} \boldsymbol{\rho}^{T}=n \mathbf{I}$. This implies $\operatorname{det} \boldsymbol{\rho} \neq 0$ and $\boldsymbol{\rho}^{-1}=(1 / n) \boldsymbol{\rho}^{T}$ so that $(1 / n) \boldsymbol{\rho}^{T} \boldsymbol{\rho}=\mathbf{I}$, i.e. the columns of the irrep table are also mutually orthogonal.

As seen in Equations 8 and 10 the complementary spaces $P_{i} \mathcal{H}(\Omega)$ collectively span $\mathcal{H}(\Omega)$, i.e. $\mathcal{H}(\Omega)=$ $\bigoplus_{i=1}^{n} P_{i} \mathcal{H}(\Omega)$. In this way we can uniquely express a function $w$ over a domain $\Omega$ with a given abelian symmetry group $\mathcal{G}$ as $w(\mathbf{x})=\sum_{i=1}^{n} P_{i} w(\mathbf{x})$ just as we use the fact that $\mathcal{R}^{n}=\operatorname{Span}\left\{\mathbf{e}_{1}, \mathbf{e}_{2}, \ldots\right\}$ to express the vector $\mathbf{x}=\sum_{i=1}^{n} x_{i} \mathbf{e}_{i}$. It is also noted that each of the $P_{i} \mathcal{H}(\Omega)$ are invariant with respect to the group actions, i.e. $w \in P_{i} \mathcal{H}(\Omega)$ if and only if $w \circ \sigma_{g} \in P_{i} \mathcal{H}(\Omega)$ for all $g \in \mathcal{G}$.

Differentiating Equations 5 and 6 and using the orthogonality of the $\mathbf{R}_{g}$ gives

$$
\begin{aligned}
\nabla P_{i} w(\mathbf{x}) & =\frac{1}{n} \sum_{g \in \mathcal{G}} \rho_{i}\left(\sigma_{g}\right) \mathbf{R}_{g} \nabla w\left(\mathbf{R}_{g}^{-1} \mathbf{x}\right) \\
\nabla P_{i} w\left(\mathbf{R}_{g}^{-1} \mathbf{x}\right) & =\rho_{i}\left(\sigma_{g}\right) \mathbf{R}_{g}^{-1} \nabla P_{i} w(\mathbf{x})
\end{aligned}
$$

Noting the vector like analogy between $\nabla w$ and $\chi$ we define and subsequently find

$$
\begin{aligned}
P_{i} \chi(\mathbf{x}) & =\frac{1}{n} \sum_{g \in \mathcal{G}} \rho_{i}\left(\sigma_{g}\right) \mathbf{R}_{g} \chi\left(\mathbf{R}_{g}^{-1} \mathbf{x}\right) \\
P_{i} \chi\left(\mathbf{R}_{g}^{-1} \mathbf{x}\right) & =\rho_{i}\left(\sigma_{g}\right) \mathbf{R}_{g}^{-1} P_{i} \chi(\mathbf{x})
\end{aligned}
$$

Arguing as we do to obtain Equation 7, it is seen that the above Equations 11 and 12 can also be stated as

$$
\begin{aligned}
\nabla P_{i} w\left(\mathbf{R}_{g} \mathbf{x}\right) & =\rho_{i}\left(\sigma_{g}\right) \mathbf{R}_{g} \nabla P_{i} w(\mathbf{x}) \\
P_{i} \chi\left(\mathbf{R}_{g} \mathbf{x}\right) & =\rho_{i}\left(\sigma_{g}\right) \mathbf{R}_{g} P_{i} \chi(\mathbf{x})
\end{aligned}
$$

We return to the integral Equation 4. Since it must hold for arbitrary $v \in \mathcal{H}(\Omega, 0)$ it must hold for some 
$v_{i}=P_{i} v$. Substituting this choice into Equation 4 gives

$$
\begin{aligned}
& \int_{\Omega} \nabla v_{i}(\mathbf{x}) \cdot \mathbf{k}(\mathbf{x}) \nabla w(\mathbf{x}) d v=\int_{\Omega}\left(\nabla v_{i}(\mathbf{x}) \cdot \mathbf{k}(\mathbf{x}) \chi(\mathbf{x})+v_{i}(\mathbf{x}) f(\mathbf{x})\right) d v+ \\
& \int_{\partial \Omega^{n}} v_{i}(\mathbf{x}) q^{p}(\mathbf{x}) d a \\
& \sum_{g \in \mathcal{G}} \int_{\mathbf{R}_{g}^{-1}(C)} \nabla v_{i}(\mathbf{x}) \cdot \mathbf{k}(\mathbf{x}) \nabla w(\mathbf{x}) d v=\sum_{g \in \mathcal{G}} \int_{\mathbf{R}_{g}^{-1}(C)}\left(\nabla v_{i}(\mathbf{x}) \cdot \mathbf{k}(\mathbf{x}) \chi(\mathbf{x})+v_{i}(\mathbf{x}) f(\mathbf{x})\right) d v+ \\
& \sum_{g \in \mathcal{G}} \int_{\mathbf{R}_{g}^{-1}\left(\partial C^{n}\right)} v_{i}(\mathbf{x}) q^{p}(\mathbf{x}) d a \\
& \sum_{g \in \mathcal{G}} \int_{C} \nabla v_{i}\left(\mathbf{R}_{g}^{-1} \mathbf{x}\right) \cdot \mathbf{k}\left(\mathbf{R}_{g}^{-1} \mathbf{x}\right) \nabla w\left(\mathbf{R}_{g}^{-1} \mathbf{x}\right) d v=\sum_{g \in \mathcal{G}} \int_{C}\left(\nabla v_{i}\left(\mathbf{R}_{g}^{-1} \mathbf{x}\right) \cdot \mathbf{k}\left(\mathbf{R}_{g}^{-1} \mathbf{x}\right) \boldsymbol{\chi}\left(\mathbf{R}_{g}^{-1} \mathbf{x}\right)+\right. \\
& \left.v_{i}\left(\mathbf{R}_{g}^{-1} \mathbf{x}\right) f\left(\mathbf{R}_{g}^{-1} \mathbf{x}\right)\right) d v+ \\
& \sum_{g \in \mathcal{G}} \int_{\partial C^{n}} v_{i}\left(\mathbf{R}_{g}^{-1} \mathbf{x}\right) q^{p}\left(\mathbf{R}_{g}^{-1} \mathbf{x}\right) d a \\
& \sum_{g \in \mathcal{G}} \int_{C} \rho_{i}\left(\sigma_{g}\right) \mathbf{R}_{g}^{-1} \nabla v_{i}(\mathbf{x}) \cdot \mathbf{R}_{g}^{-1} \mathbf{k}(\mathbf{x}) \mathbf{R}_{g} \nabla w\left(\mathbf{R}_{g}^{-1} \mathbf{x}\right) d v \quad=\sum_{g \in \mathcal{G}} \int_{C}\left(\rho_{i}\left(\sigma_{g}\right) \mathbf{R}_{g}^{-1} \nabla v_{i}(\mathbf{x}) \cdot \mathbf{R}_{g}^{-1} \mathbf{k}(\mathbf{x}) \mathbf{R}_{g} \chi\left(\mathbf{R}_{g}^{-1} \mathbf{x}\right)+\right. \\
& \left.\rho_{i}\left(\sigma_{g}\right) v_{i}(\mathbf{x}) f\left(\mathbf{R}_{g}^{-1} \mathbf{x}\right)\right) d v+ \\
& \sum_{g \in \mathcal{G}} \int_{\partial C^{n}} \rho_{i}\left(\sigma_{g}\right) v_{i}(\mathbf{x}) q^{p}\left(\mathbf{R}_{g}^{-1} \mathbf{x}\right) d a \\
& \sum_{g \in \mathcal{G}} \int_{C} \nabla v_{i}(\mathbf{x}) \cdot \mathbf{k}(\mathbf{x}) \rho_{i}\left(\sigma_{g}\right) \mathbf{R}_{g} \nabla w\left(\mathbf{R}_{g}^{-1} \mathbf{x}\right) d v=\sum_{g \in \mathcal{G}} \int_{C}\left(\nabla v_{i}(\mathbf{x}) \cdot \mathbf{k}(\mathbf{x}) \rho_{i}\left(\sigma_{g}\right) \mathbf{R}_{g} \chi\left(\mathbf{R}_{g}^{-1} \mathbf{x}\right)+\right. \\
& \left.v_{i}(\mathbf{x}) \rho_{i}\left(\sigma_{g}\right) f\left(\mathbf{R}_{g}^{-1} \mathbf{x}\right)\right) d v+ \\
& \sum_{g \in \mathcal{G}} \int_{\partial C^{n}} v_{i}(\mathbf{x}) \rho_{i}\left(\sigma_{g}\right) q^{p}\left(\mathbf{R}_{g}^{-1} \mathbf{x}\right) d a \\
& n \int_{C} \nabla v_{i}(\mathbf{x}) \cdot \mathbf{k}(\mathbf{x}) \nabla w_{i}(\mathbf{x}) d v=n \int_{C}\left(\nabla v_{i}(\mathbf{x}) \cdot \mathbf{k}(\mathbf{x}) \boldsymbol{\chi}_{i}(\mathbf{x})+\right. \\
& \left.v_{i}(\mathbf{x}) f_{i}(\mathbf{x})\right) d v+ \\
& n \int_{\partial C^{n}} v_{i}(\mathbf{x}) q_{i}^{p}(\mathbf{x}) d a
\end{aligned}
$$

where it is understood that, e.g. $w_{i}=P_{i} w$. To obtain the above we used the summation of integration, change-of-variable theorem, Equations $11-12$ and the domain symmetry of $\mathbf{k}$.

As seen above, the projection property is such that our choice of $v_{i}$ renders an integral expression which only contains the $w_{i}$ component of $w$. So by using Equation 9 to express $v=\sum_{i=1}^{n} v_{i}$ and substituting into Equation 4 we are able to evaluate the response $w=\sum_{i=1}^{n} w_{i}$ one component at a time and that is exactly what we do. In light of this the boundary value problem of Equation 4 is now converted into $n$ problems which read: find the response $w_{i} \in \mathcal{H}\left(C, w_{i}^{p}\right)$ such that

$$
\int_{C} \nabla v_{i} \cdot \mathbf{k} \nabla w_{i} d v=\int_{C}\left(\nabla v_{i} \cdot \mathbf{k} \boldsymbol{\chi}_{i}+v_{i} f_{i}\right) d v+\int_{\partial C^{n}} v_{i} q_{i}^{p} d a
$$

for all $v_{i} \in \mathcal{H}(C, 0)$. The obvious benefit that these problems offer is the replacement of one problem defined over the large domain $\Omega$ by $n$ problems defined over the smaller symmetry cell $C$.

The above result is not yet satisfactory because we have not incorporated the boundary conditions on the "cut" surfaces of $C$, i.e. the boundary $\Sigma=\partial C \backslash\left(\partial C^{d} \cup \partial C^{n}\right)$ of $C$ that does not coincide with the boundaries $\partial \Omega^{d}$ and $\partial \Omega^{n}$. E.g. referring to the $D_{2}$ domain of Figure 2 , a point $\mathbf{x} \in C$ is on $\Sigma_{x}$ if $\mathbf{x}=\mathbf{R}_{x} \mathbf{x}$. ${ }^{2}$ And since we allow neither jumps in the response nor in the flux throughout the domain, these points must satisfy

$$
\begin{aligned}
\llbracket w_{i}(\mathbf{x}) \rrbracket & =0 \\
\llbracket \mathbf{k}(\mathbf{x})\left(\nabla w_{i}(\mathbf{x})-\chi_{i}(\mathbf{x})\right) \rrbracket \cdot \mathbf{n}(\mathbf{x}) & =0
\end{aligned}
$$

\footnotetext{
${ }^{2}$ The equality $\mathbf{x}=\mathbf{R}_{g} \mathbf{x}$ is not always satisfied for points on cut boundaries $\Sigma_{g}$. We will comment more on this later, cf. Footnote 10.
} 
Considering $D_{2}$, Equations 7 and 13 and the above jump conditions over $\Sigma_{x}$ give

$$
\begin{aligned}
w_{i}(\mathbf{x}) & =w_{i}\left(\mathbf{R}_{x} \mathbf{x}\right) \\
& =\rho_{i}\left(\sigma_{x}\right) w_{i}(\mathbf{x})
\end{aligned}
$$

and

$$
\begin{aligned}
\mathbf{k}(\mathbf{x})\left(\nabla w_{i}(\mathbf{x})-\chi_{i}(\mathbf{x})\right) \cdot \mathbf{n}(\mathbf{x}) & =\mathbf{k}\left(\mathbf{R}_{x} \mathbf{x}\right)\left(\nabla w_{i}\left(\mathbf{R}_{x} \mathbf{x}\right)-\boldsymbol{\chi}_{i}\left(\mathbf{R}_{x} \mathbf{x}\right)\right) \cdot \mathbf{n}(\mathbf{x}) \\
& =\mathbf{R}_{x} \mathbf{k}(\mathbf{x}) \mathbf{R}_{x}^{-1} \rho_{i}\left(\sigma_{x}\right) \mathbf{R}_{x}\left(\nabla w_{i}(\mathbf{x})-\boldsymbol{\chi}_{i}(\mathbf{x})\right) \cdot \mathbf{n}(\mathbf{x}) \\
& =\rho_{i}\left(\sigma_{x}\right) \mathbf{k}(\mathbf{x})\left(\nabla w_{i}(\mathbf{x})-\boldsymbol{\chi}_{i}(\mathbf{x})\right) \cdot \mathbf{R}_{x}^{T} \mathbf{n}(\mathbf{x}) \\
& =-\rho_{i}\left(\sigma_{x}\right) \mathbf{k}(\mathbf{x})\left(\nabla w_{i}(\mathbf{x})-\boldsymbol{\chi}_{i}(\mathbf{x})\right) \cdot \mathbf{n}(\mathbf{x})
\end{aligned}
$$

where we use the fact that $\mathbf{R}_{x}^{T} \mathbf{n}(\mathbf{x})=-\mathbf{n}(\mathbf{x})$ for $\mathbf{x} \in \Sigma_{x}$. The above Equations 16 and 17 can be equivalently expressed as

$$
\begin{aligned}
& \frac{1}{2}\left(1-\rho_{i}\left(\sigma_{x}\right)\right) w_{i}(\mathbf{x})=0 \\
& \frac{1}{2}\left(1+\rho_{i}\left(\sigma_{x}\right)\right) q_{i}(\mathbf{x})=0
\end{aligned}
$$

where $q_{i}(\mathbf{x})=\mathbf{k}(\mathbf{x})\left(\nabla w_{i}(\mathbf{x})-\boldsymbol{\chi}_{i}(\mathbf{x})\right) \cdot \mathbf{n}(\mathbf{x})$ is the normal flux. Note that $1 / 2\left(1-\rho_{i}\left(\sigma_{x}\right)\right)$ and $1 / 2\left(1+\rho_{i}\left(\sigma_{x}\right)\right)$ are projections in the sense that, e.g. $1 / 2\left(1-\rho_{i}\left(\sigma_{x}\right)\right) \times 1 / 2\left(1-\rho_{i}\left(\sigma_{x}\right)\right)=1 / 2\left(1-\rho_{i}\left(\sigma_{x}\right)\right)$; moreover they are mutually orthogonal projections, i.e. $1 / 2\left(1-\rho_{i}\left(\sigma_{x}\right)\right) \times 1 / 2\left(1+\rho_{i}\left(\sigma_{x}\right)\right)=0$ and complementary, i.e. $1 / 2\left(1-\rho_{i}\left(\sigma_{x}\right)\right)+1 / 2\left(1+\rho_{i}\left(\sigma_{x}\right)\right)=1$. Thusly, the Dirichlet and Neumann boundary conditions on the cut surfaces can be thought of as orthogonal complements. ${ }^{3}$ For $i=1,2$ we have $\rho_{i}\left(\sigma_{x}\right)=1$ so the second equation above requires $q_{i}(\mathbf{x})=0$. Whereas for $i=3,4$ we have $\rho_{i}\left(\sigma_{x}\right)=-1$ so that the first equation above requires $w_{i}(\mathbf{x})=0$. Performing similar derivation for $\mathbf{x} \in \Sigma_{y}$ we find $w_{i}(\mathbf{x})=0$ for $i=2,4$ and $q_{i}(\mathbf{x})=0$ for $i=1,3$.

Summarizing, for the $D_{2}$ symmetric domain we solve $n=4$ boundary value problems on $C$ for the $w_{i}$ of the form

$$
\begin{aligned}
& \operatorname{div}\left(\mathbf{k}\left(\nabla w_{i}-\chi_{i}\right)+f_{i}=0 \quad \text { in } C \text { and } i=1,2,3,4\right. \\
& w_{i}=w_{i}^{p} \text { on } \partial C^{d} \text { and } i=1,2,3,4 \\
& q_{i}=q_{i}^{p} \quad \text { on } \partial C^{n} \text { and } i=1,2,3,4 \\
& \left\{\begin{array}{l}
q_{1} \\
q_{2} \\
w_{3} \\
w_{4}
\end{array}\right\}=\mathbf{0} \text { on } \Sigma_{x} \\
& \left\{\begin{array}{c}
q_{1} \\
w_{2} \\
q_{3} \\
w_{4}
\end{array}\right\}=\mathbf{0} \quad \text { on } \Sigma_{y}
\end{aligned}
$$

In the above we see that the applied flux $q_{i}=0$ for $i=1,2$ on $\Sigma_{x}$ and for $i=1,3$ on $\Sigma_{y}$. This Neumann boundary condition is automatically accommodated in the the weak formulation, cf. Equation 15.

These responses $w_{i}$ obtained by solving the above equations are used to obtain $w_{i}$ throughout $\Omega$ via Equation 7 and summed via Equation 9 to evaluate $w$, i.e.

$$
w\left(\mathbf{R}_{g} \mathbf{x}\right)=\sum_{i=1}^{n} w_{i}\left(\mathbf{R}_{g} \mathbf{x}\right)=\sum_{i=1}^{n} \rho_{i}\left(\sigma_{g}\right) w_{i}(\mathbf{x}),
$$

where $\mathbf{x} \in C$.

\footnotetext{
${ }^{3}$ This will be more obvious in the subsequent discussions.
} 


\subsection{Homogenization under $D_{2}$ symmetry}

Figure 2 represents any $D_{2}$ symmetric domain. We now consider the $D_{2}$ symmetric unit cell of Figure 3 for the purpose of homogenization. The primary difference between the homogenization analysis discussed here and the "usual" analysis presented above is that we now must enforce periodic boundary conditions.
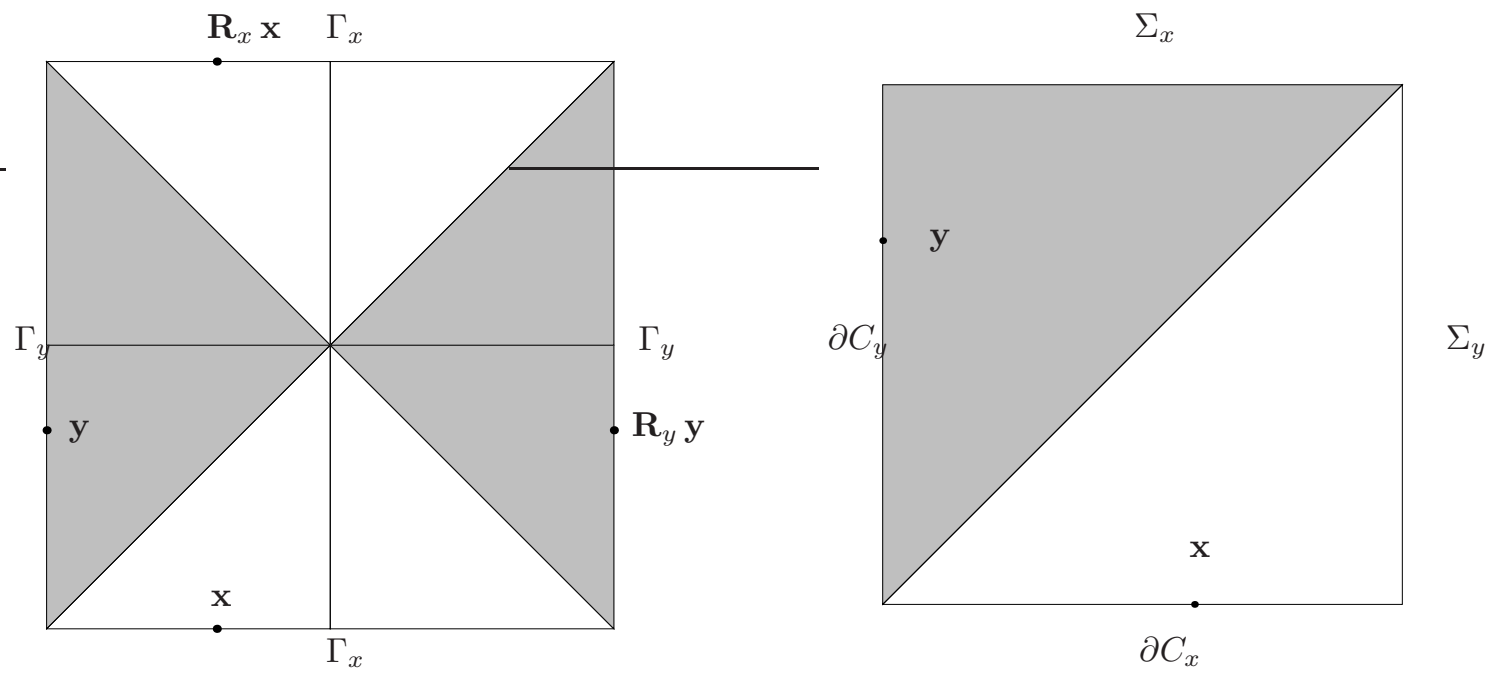

Figure 3: $D_{2}$ unit cell $\Omega$ (left) and its symmetry cell $C$ (right).

Periodic homogenization theory deals with bodies exhibiting a periodically repeated pattern [12]. To facilitate the analysis of such bodies, the response over the microscopic periodically cells is homogenized such that the homogenized macroscopic material behavior is the same as that of the original body with its fine-scale features in an energetic sense. To obtain the homogenized macroscopic material behavior we solve several elliptic problems on the periodicity cell and then integrate functions of these analyzes' responses over periodicity cell.

The square periodicity considered in this subsection is described by two translations ${ }^{4}$ which we shall denote by $\tau_{1}$ and $\tau_{2}$, defined as

$$
\begin{array}{lll}
\tau_{1}(\mathbf{x})=\mathbf{x}+\mathbf{e}_{1} & \text { where } & \mathbf{e}_{1}=\left[\begin{array}{ll}
1 & 0
\end{array}\right]^{T} \\
\tau_{2}(\mathbf{x})=\mathbf{x}+\mathbf{e}_{2} & \text { where } & \mathbf{e}_{2}=\left[\begin{array}{ll}
0 & 1
\end{array}\right]^{T}
\end{array}
$$

The state function $w$ must be invariant to these translations, that is, $w\left(\tau_{i}(\mathbf{x})\right)=x(\mathbf{x})$ for all $\mathbf{x}$ and $i=1,2$. Now we "cut" a periodicity cell $\Omega$ from the larger body and we allow neither jumps in the response nor in the flux across its boundary $\partial \Omega$; the resulting boundary conditions are twofold. On one hand, $w$ must take equal values on "opposite faces" of the cell $\Omega .{ }^{5}$ On the other hand, the flux $q$ must take equal values with opposite signs because the normal vectors $\mathbf{n}(\mathbf{x})$ and $\mathbf{n}\left(\tau_{i}(\mathbf{x})\right)$ have opposite directions.

Since we have chosen a cell $\Omega$ centered at the origin, we can safely replace the translation $\tau_{1}$ by the reflection $\mathbf{R}_{y}$ and $\tau_{2}$ by $\mathbf{R}_{x}$, thus expressing the boundary conditions in terms of reflections only. So, for $\mathbf{x} \in \partial C_{x}=\partial C \cap \Gamma_{x}$ we require that $w\left(\mathbf{R}_{x} \mathbf{x}\right)=w(\mathbf{x})$ and $q(\mathbf{x})=-q\left(\mathbf{R}_{x} \mathbf{x}\right)$. Similarly, for $\mathbf{y} \in \partial C_{y}=\partial C \cap \Gamma_{y}$ we require that $w\left(\mathbf{R}_{y} \mathbf{y}\right)=w(\mathbf{y})$ and $q(\mathbf{y})=-q\left(\mathbf{R}_{y} \mathbf{y}\right)$.

For the first case with $\mathbf{x} \in \partial C_{x}$, periodicity and Equation 7 require

$$
\begin{aligned}
w_{i}(\mathbf{x}) & =w_{i}\left(\mathbf{R}_{x} \mathbf{x}\right) \\
& =\rho_{i}\left(\sigma_{x}\right) w_{i}(\mathbf{x})
\end{aligned}
$$

The above implies $w_{3}(\mathbf{x})=w_{4}(\mathbf{x})=0$ since $\rho_{3}\left(\sigma_{x}\right)=\rho_{4}\left(\sigma_{x}\right)=-1$ and does not provide any insight on $w_{1}$ and $w_{2}$ since $\rho_{1}\left(\sigma_{x}\right)=\rho_{2}\left(\sigma_{x}\right)=1$. For the flux condition $q(\mathbf{x})=-q\left(\mathbf{R}_{x} \mathbf{x}\right)$ we apply a reasoning similar to

\footnotetext{
${ }^{4}$ More precisely, the body under study is invariant to a group of translations; $\tau_{1}$ and $\tau_{2}$ are two generators of this group.

${ }^{5}$ By "opposite faces" we mean faces wich relate to each other through $\tau_{1}$ or $\tau_{2}$.
} 
Equation 17 (note that $\mathbf{n}\left(\mathbf{R}_{x} \mathbf{x}\right)=\mathbf{R}_{x} \mathbf{n}(\mathbf{x})=-\mathbf{n}(\mathbf{x})$ ) and obtain

$$
\mathbf{k}(\mathbf{x})\left(\nabla w_{i}(\mathbf{x})-\chi_{i}(\mathbf{x})\right) \cdot \mathbf{n}(\mathbf{x})=-\rho_{i}\left(\sigma_{x}\right) \mathbf{k}(\mathbf{x})\left(\nabla w_{i}(\mathbf{x})-\chi_{i}(\mathbf{x})\right) \cdot \mathbf{n}(\mathbf{x})
$$

which implies $q_{1}(\mathbf{x})=q_{2}(\mathbf{x})=0$ since $\rho_{1}\left(\sigma_{x}\right)=\rho_{2}\left(\sigma_{x}\right)=1$ and gives no information on $q_{3}$ and $q_{4}$ since $\rho_{3}\left(\sigma_{x}\right)=\rho_{4}\left(\sigma_{x}\right)=-1$.

Similarly, for $\mathbf{y} \in \partial C_{y}$, periodicity requires $w_{2}(\mathbf{y})=w_{4}(\mathbf{y})=0$ since $\rho_{2}\left(\sigma_{y}\right)=\rho_{4}\left(\sigma_{y}\right)=-1$ while the flux condition implies $q_{1}(\mathbf{y})=q_{3}(\mathbf{y})=0$ since $\rho_{1}\left(\sigma_{y}\right)=\rho_{3}\left(\sigma_{y}\right)=1$.

The "unit loads" for homogenization are $\chi^{(1)}=\mathbf{e}_{1}=\left[\begin{array}{ll}1 & 0\end{array}\right]^{T}$ and $\chi^{(2)}=\mathbf{e}_{2}=\left[\begin{array}{ll}0 & 1\end{array}\right]^{T}$. Breaking these down into their "components" via Equation 12 gives

$$
\begin{array}{lll}
\chi_{1}^{(1)}=\chi_{3}^{(1)}=\chi_{4}^{(1)}=\mathbf{0} & \text { and } & \chi_{2}^{(1)}=\chi^{(1)}=\mathbf{e}_{1} \\
\chi_{1}^{(2)}=\chi_{2}^{(2)}=\chi_{4}^{(2)}=\mathbf{0} & \text { and } & \chi_{3}^{(2)}=\chi^{(2)}=\mathbf{e}_{2}
\end{array}
$$

The null load cases and Equation 9 imply $w^{(1)}=w_{2}^{(1)}$ and $w^{(2)}=w_{3}^{(2)}$.

The two unit cell problems on the large domain $\Omega$ are thusly reduced to two unit cell problems on the symmetry cell $C$ which do not require the enforcement of periodicity. The first problem is to find $w^{(1)}=w_{2}^{(1)}$ such that

$$
\begin{array}{rll}
\operatorname{div}\left(\mathbf{k}\left(\nabla w^{(1)}-\mathbf{e}_{1}\right)\right. & =0 & \text { in } C \\
q^{(1)} & =0 & \text { on } \Sigma_{x} \cup \partial C_{x} \\
w^{(1)} & =0 & \text { on } \Sigma_{y} \cup \partial C_{y}
\end{array}
$$

whereas the second problem is to find $w^{(2)}=w_{3}^{(2)}$ such that

$$
\begin{array}{rll}
\operatorname{div}\left(\mathbf{k}\left(\nabla w^{(2)}-\mathbf{e}_{2}\right)\right. & =0 & \text { in } C \\
w^{(2)} & =0 & \text { on } \Sigma_{x} \cup \partial C_{x} \\
q^{(2)} & =0 & \text { on } \Sigma_{y} \cup \partial C_{y}
\end{array}
$$

The finite element approximations for $w^{(1)}$ and $w^{(2)}$ require the evaluations and factorizations of two stiffness matrices, however these matrices correspond to the small symmetry cell $C$, not the full domain $\Omega$. And, as described at the end of Section 2.3, it is possible to evaluate and factor a single stiffness matrix on the symmetry sector if we accommodate the $w^{(i)}=0$ boundary conditions via constraints.

The homogenized conductivity tensor $\mathbf{k}_{h}$ is evaluated with the help of Equation 13, the change of variable theorem, the domain symmetry of $\mathbf{k}$ and the equalities $w^{(1)}=w_{2}^{(1)}$ and $w^{(2)}=w_{3}^{(2)}$, i.e.

$$
\begin{aligned}
\mathbf{k}_{h} & =\frac{1}{\operatorname{vol}(\Omega)} \int_{\Omega} \mathbf{k}(\mathbf{x})\left(\mathbf{I}+\nabla w^{(1)}(\mathbf{x}) \otimes \mathbf{e}_{1}+\nabla w^{(2)}(\mathbf{x}) \otimes \mathbf{e}_{2}\right) d a \\
& =\frac{1}{\operatorname{vol}(\Omega)} \int_{\Omega} \mathbf{k}(\mathbf{x})\left(\mathbf{I}+\nabla w_{2}^{(1)}(\mathbf{x}) \otimes \mathbf{e}_{1}+\nabla w_{3}^{(2)}(\mathbf{x}) \otimes \mathbf{e}_{2}\right) d a \\
& =\sum_{g} \frac{1}{\operatorname{vol}(\Omega)} \int_{C} \mathbf{k}\left(\mathbf{R}_{g} \mathbf{x}\right)\left(\mathbf{I}+\nabla w_{2}^{(1)}\left(\mathbf{R}_{g} \mathbf{x}\right) \otimes \mathbf{e}_{1}+\nabla w_{3}^{(2)}\left(\mathbf{R}_{g} \mathbf{x}\right) \otimes \mathbf{e}_{2}\right) d a \\
& =\sum_{g} \frac{1}{\operatorname{vol}(\Omega)} \int_{C} \mathbf{R}_{g} \mathbf{k}(\mathbf{x}) \mathbf{R}_{g}^{-1}\left(\mathbf{I}+\mathbf{R}_{g}\left[\rho_{2}\left(\sigma_{g}\right) \nabla w_{2}^{(1)}(\mathbf{x}) \otimes \mathbf{e}_{1}+\rho_{3}\left(\sigma_{g}\right) \nabla w_{3}^{(2)}(\mathbf{x}) \otimes \mathbf{e}_{2}\right]\right) d a \\
& =\sum_{g} \frac{1}{\operatorname{vol}(\Omega)} \int_{C} \mathbf{R}_{g} \mathbf{k}(\mathbf{x})\left(\mathbf{R}_{g}^{T}+\rho_{2}\left(\sigma_{g}\right) \nabla w_{2}^{(1)}(\mathbf{x}) \otimes \mathbf{e}_{1}+\rho_{3}\left(\sigma_{g}\right) \nabla w_{1}^{(2)}(\mathbf{x}) \otimes \mathbf{e}_{2}\right) d a
\end{aligned}
$$

\subsection{Nonabelian symmetry}

Now we discuss the more complicated nonabelian problem in the context of $D_{3}$ symmetric domains, cf. Figure 4, i.e. domains that are symmetric with respect to the identity $\sigma_{1}, 2 \pi / 3$ and $4 \pi / 3$ rotations about the $z$-axis $\sigma_{2}$ and $\sigma_{4}$ and reflections about the $y$ axis of the aforementioned, i.e. $\sigma_{y}, \sigma_{y 2}$ and $\sigma_{y 4}$ so that 
$\mathcal{G}=\left\{\sigma_{1}, \sigma_{2}, \sigma_{4}, \sigma_{y}, \sigma_{y 2}, \sigma_{y 4}\right\}$ is order $n=6 .{ }^{6}$ As just stated, this is more complicated than the previous abelian symmetry, albeit $D_{3}$ is the simplest of the nonabelian symmetries.

The (new) group elements $\sigma_{g}$ are tabulated below with their transformations $\mathbf{R}_{g}$ in row 2 and their respective inverses $\mathbf{R}_{g}^{-1}$ in row 3

\begin{tabular}{|c|c|c|c|c|c|c|c|c|c|c|}
\hline$\sigma_{g}$ & $\sigma_{1}$ & \multicolumn{2}{|r|}{$\sigma_{2}$} & \multicolumn{2}{|r|}{$\sigma_{4}$} & $\sigma_{y}$ & \multicolumn{2}{|c|}{$\sigma_{y 2}$} & \multicolumn{2}{|r|}{$\sigma_{y 4}$} \\
\hline $\mathbf{R}_{g}$ & $\mathbf{R}_{1}$ & $\mathbf{R}_{2}=$ & $\begin{array}{cc}-\frac{1}{2} & -\frac{\sqrt{3}}{2} \\
\frac{\sqrt{3}}{2} & -\frac{1}{2}\end{array}$ & $\mathbf{R}_{4}=$ & $\begin{array}{cc}-\frac{1}{2} & \frac{\sqrt{3}}{2} \\
-\frac{\sqrt{3}}{2} & -\frac{1}{2}\end{array}$ & $\mathbf{R}_{y}$ & $\mathbf{R}_{y 2}=$ & $\begin{array}{ll}\frac{1}{2} & \frac{\sqrt{3}}{2} \\
\frac{\sqrt{3}}{2} & -\frac{1}{2}\end{array}$ & $\mathbf{R}_{y 4}=$ & $\begin{array}{cc}\frac{1}{2} & -\frac{\sqrt{3}}{2} \\
-\frac{\sqrt{3}}{2} & -\frac{1}{2}\end{array}$ \\
\hline$\overline{\mathbf{R}_{g}^{-1}}$ & $\mathbf{R}_{1}$ & & $\mathbf{R}_{4}$ & & $\mathbf{R}_{2}$ & $\overline{\mathbf{R}_{y}}$ & & $\overline{\mathbf{R}_{y 2}}$ & & $\mathbf{R}_{y 4}$ \\
\hline
\end{tabular}

It can readily be verified that these transformations are orthogonal. The group multiplication table for $D_{3}$ is given by

\begin{tabular}{|c||c|c|c|c|c|c|}
\hline & $\sigma_{1}$ & $\sigma_{2}$ & $\sigma_{4}$ & $\sigma_{y}$ & $\sigma_{y 2}$ & $\sigma_{y 4}$ \\
\hline \hline$\sigma_{1}$ & $\sigma_{1}$ & $\sigma_{2}$ & $\sigma_{4}$ & $\sigma_{y}$ & $\sigma_{y 2}$ & $\sigma_{y 4}$ \\
\hline$\sigma_{2}$ & $\sigma_{2}$ & $\sigma_{4}$ & $\sigma_{1}$ & $\sigma_{y 4}$ & $\sigma_{y}$ & $\sigma_{y 2}$ \\
\hline$\sigma_{4}$ & $\sigma_{4}$ & $\sigma_{1}$ & $\sigma_{2}$ & $\sigma_{y 2}$ & $\sigma_{y 4}$ & $\sigma_{y}$ \\
\hline$\sigma_{y}$ & $\sigma_{y}$ & $\sigma_{y 2}$ & $\sigma_{y 4}$ & $\sigma_{1}$ & $\sigma_{2}$ & $\sigma_{4}$ \\
\hline$\sigma_{y 2}$ & $\sigma_{y 2}$ & $\sigma_{y 4}$ & $\sigma_{y}$ & $\sigma_{4}$ & $\sigma_{1}$ & $\sigma_{2}$ \\
\hline$\sigma_{y 4}$ & $\sigma_{y 4}$ & $\sigma_{y}$ & $\sigma_{y 2}$ & $\sigma_{2}$ & $\sigma_{4}$ & $\sigma_{1}$ \\
\hline
\end{tabular}

Again note that each element of the group appears once in each row of the table. For this nonabelian group the row/column order is consequential since the composition operation does not commute, e.g. $\sigma_{4} \sigma_{y 2}=$ $\sigma_{y 4} \neq \sigma_{y 2} \sigma_{4}=\sigma_{y}$.
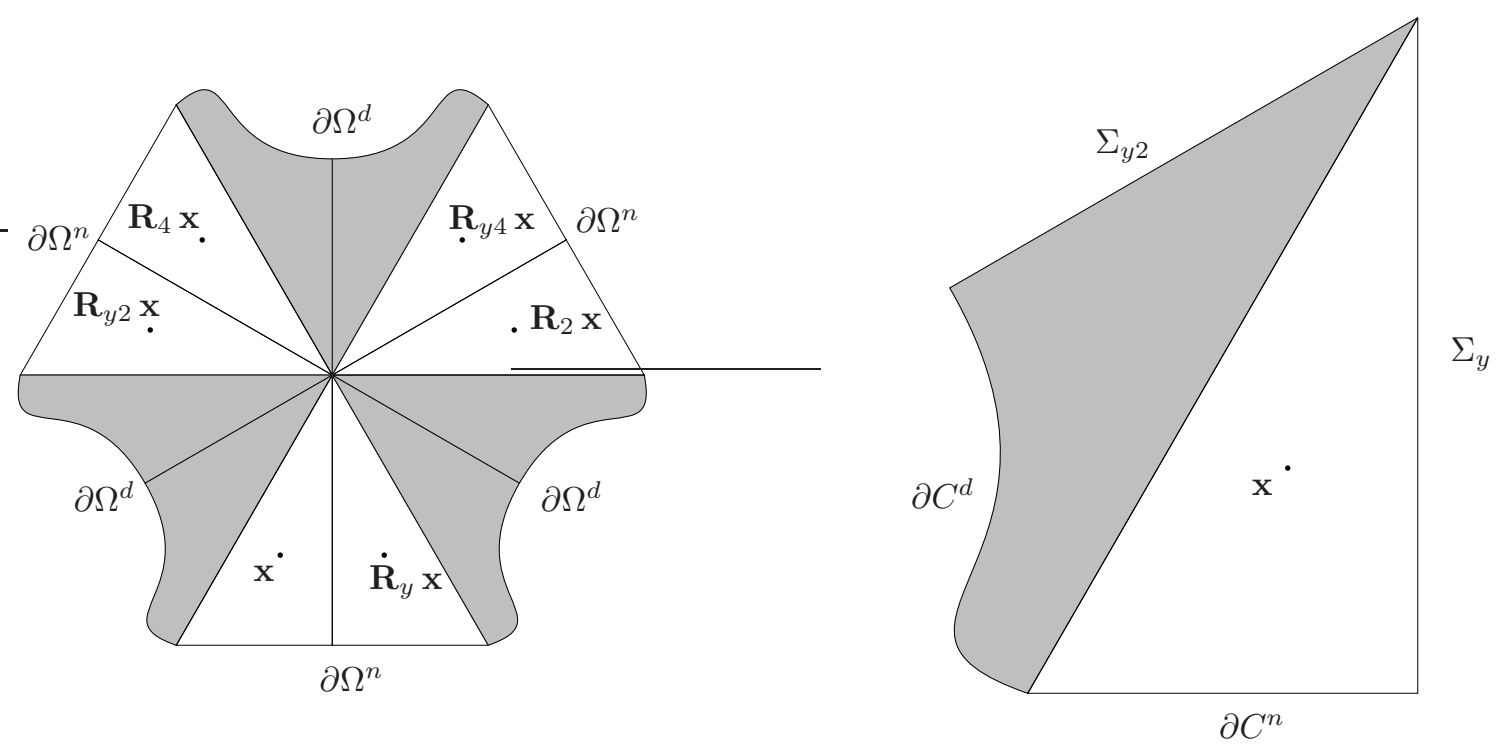

Figure 4: $D_{3}$ symmetric domain $\Omega$ (left) and symmetry cell $C$ (right).

\footnotetext{
${ }^{6}$ Equivalently we have the three rotational symmetries: $2 \pi, 2 \pi / 3$ and $4 \pi / 3$ about the $z$-axis, i.e. $\sigma_{1}, \sigma_{2}$ and $\sigma_{4}$ and the three reflective symmetries: about the $y$ axis and the two lines that pass through the origin and 2 and 4 o'clock, i.e. $\sigma_{y}, \sigma_{y 2}$ and $\sigma_{y 4}$.
} 
The irreps table for $D_{3}$ is given by

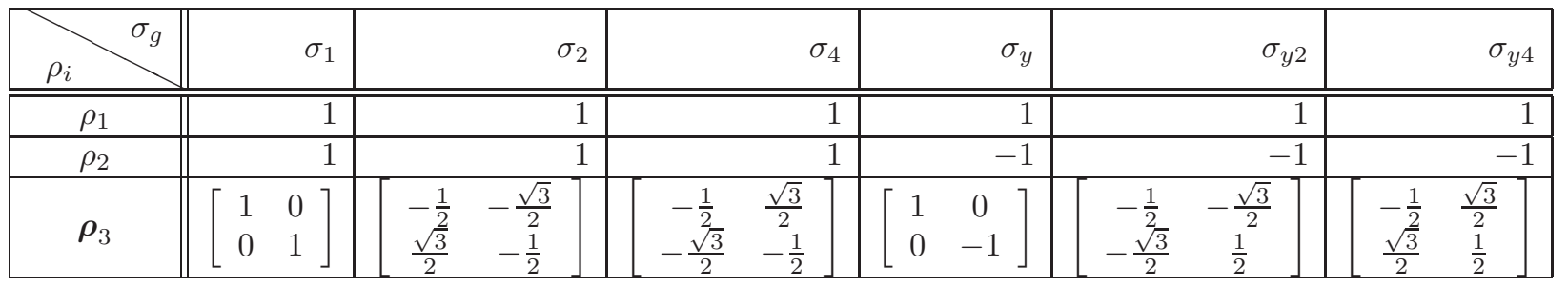

Things are different for the nonabelian case. Notably we have the matrix valued irrep $\boldsymbol{\rho}_{3}$. In the general nonabelian case, as exemplified above, we note that 1) $\boldsymbol{\rho}_{i}\left(\sigma_{1}\right)=\operatorname{diag}(1,1, \cdots)$ is an $d_{i} \times d_{i}$ identity matrix where $d_{i}$ is the dimension of the representation matrix $\left.\boldsymbol{\rho}_{i}, 2\right)$ the representations are orthogonal, i.e. $\boldsymbol{\rho}_{i}\left(\sigma_{l}^{-1}\right)=$ $\left[\boldsymbol{\rho}_{i}\left(\sigma_{l}\right)\right]^{T}$, e.g. $\left.\boldsymbol{\rho}_{3}\left(\sigma_{2}^{-1}\right)=\boldsymbol{\rho}_{3}\left(\sigma_{4}\right)=\left[\boldsymbol{\rho}_{3}\left(\sigma_{2}\right)\right]^{T}, 3\right)$ the rows are mutually orthogonal if we treat the matrices element-by-element, e.g the "1-2 row" associated $\rho_{3}^{12}$ contains $\left[\begin{array}{llllll}0 & -\frac{\sqrt{3}}{2} & \frac{\sqrt{3}}{2} & 0 & -\frac{\sqrt{3}}{2} & \frac{\sqrt{3}}{2}\end{array}\right]$, 4) the magnitude of the row squared equals $n / d_{i}$, e.g. for the $\rho_{3}^{12}$ row we see that $0^{2}+\left(-\frac{\sqrt{3}}{2}\right)^{2}+\left(\frac{\sqrt{3}}{2}\right)^{2}+0^{2}+$ $\left(-\frac{\sqrt{3}}{2}\right)^{2}+\left(\frac{\sqrt{3}}{2}\right)^{2}=6 / 2=3$ and 5$)$ the products of the irreps follow those of the group multiplication table, e.g. $\boldsymbol{\rho}_{3}\left(\sigma_{2} \sigma_{y 2}\right)=\boldsymbol{\rho}_{3}\left(\sigma_{y}\right)=\boldsymbol{\rho}_{3}\left(\sigma_{2}\right) \boldsymbol{\rho}_{3}\left(\sigma_{y 2}\right)$. Generalizing this last result yields

$$
\boldsymbol{\rho}_{i}\left(\sigma_{l} \sigma_{m}\right)=\boldsymbol{\rho}_{i}\left(\sigma_{l}\right) \boldsymbol{\rho}_{i}\left(\sigma_{m}\right)
$$

or

$$
\rho_{i}^{j k}\left(\sigma_{l} \sigma_{m}\right)=\sum_{m=1}^{d_{i}} \rho_{i}^{j m}\left(\sigma_{l}\right) \rho_{i}^{m k}\left(\sigma_{m}\right)
$$

The table is not unique for the nonabelian case because the $\boldsymbol{\rho}_{i}$ matrices can be replaced by any set of matrices related by a similarity transform, i.e. the matrix elements $\boldsymbol{\rho}_{3}\left(\sigma_{g}\right)$ can be replaced by $\mathbf{T} \boldsymbol{\rho}_{3}\left(\sigma_{g}\right) \mathbf{T}^{-1}$ for any T.

We now proceed to trace the steps taken in the abelian discussion. For $D_{3}$ the six $(n=6)$ projections are given by

$$
\begin{aligned}
P_{1} w(\mathbf{x}) & =\frac{1}{6}\left(w(\mathbf{x})+\mathrm{w}\left(\mathbf{R}_{2}^{-1} \mathbf{x}\right)+w\left(\mathbf{R}_{4}^{-1} \mathbf{x}\right)+w\left(\mathbf{R}_{y}^{-1} \mathbf{x}\right)+w\left(\mathbf{R}_{y 2}^{-1} \mathbf{x}\right)+w\left(\mathbf{R}_{y 4}^{-1} \mathbf{x}\right)\right) \\
P_{2} w(\mathbf{x}) & =\frac{1}{6}\left(w(\mathbf{x})+w\left(\mathbf{R}_{2}^{-1} \mathbf{x}\right)+w\left(\mathbf{R}_{4}^{-1} \mathbf{x}\right)-w\left(\mathbf{R}_{y}^{-1} \mathbf{x}\right)-w\left(\mathbf{R}_{y 2}^{-1} \mathbf{x}\right)-w\left(\mathbf{R}_{y 4}^{-1} \mathbf{x}\right)\right) \\
P_{3}^{11} w(\mathbf{x}) & =\frac{1}{3}\left(w(\mathbf{x})-\frac{w\left(\mathbf{R}_{2}^{-1} \mathbf{x}\right)}{2}-\frac{w\left(\mathbf{R}_{4}^{-1} \mathbf{x}\right)}{2}+w\left(\mathbf{R}_{y}^{-1} \mathbf{x}\right)-\frac{w\left(\mathbf{R}_{y 2}^{-1} \mathbf{x}\right)}{2}-\frac{w\left(\mathbf{R}_{y 4}^{-1} \mathbf{x}\right)}{2}\right) \\
P_{3}^{12} w(\mathbf{x}) & =\frac{1}{3}\left(-\frac{\sqrt{3} w\left(\mathbf{R}_{2}^{-1} \mathbf{x}\right)}{2}+\frac{\sqrt{3} w\left(\mathbf{R}_{4}^{-1} \mathbf{x}\right)}{2}-\frac{\sqrt{3} w\left(\mathbf{R}_{y 2}^{-1} \mathbf{x}\right)}{2}+\frac{\sqrt{3} w\left(\mathbf{R}_{y 4}^{-1} \mathbf{x}\right)}{2}\right) \\
P_{3}^{21} w(\mathbf{x}) & =\frac{1}{3}\left(\frac{\sqrt{3} w\left(\mathbf{R}_{2}^{-1} \mathbf{x}\right)}{2}-\frac{\sqrt{3} w\left(\mathbf{R}_{4}^{-1} \mathbf{x}\right)}{2}-\frac{\sqrt{3} w\left(\mathbf{R}_{y 2}^{-1} \mathbf{x}\right)}{2}+\frac{\sqrt{3} w\left(\mathbf{R}_{y 4}^{-1} \mathbf{x}\right)}{2}\right) \\
P_{3}^{22} w(\mathbf{x}) & =\frac{1}{3}\left(w(\mathbf{x})-\frac{w\left(\mathbf{R}_{2}^{-1} \mathbf{x}\right)}{2}-\frac{w\left(\mathbf{R}_{4}^{-1} \mathbf{x}\right)}{2}-w\left(\mathbf{R}_{y}^{-1} \mathbf{x}\right)+\frac{w\left(\mathbf{R}_{y 2}^{-1} \mathbf{x}\right)}{2}+\frac{w\left(\mathbf{R}_{y 4}^{-1} \mathbf{x}\right)}{2}\right)
\end{aligned}
$$

The weights again come from the "rows" of the above irrep table. Generalizing the above (for nonabelian groups) gives

$$
P_{i}^{j k} w(\mathbf{x})=\frac{d_{i}}{n} \sum_{g \in \mathcal{G}} \rho_{i}^{j k}\left(\sigma_{g}\right) w\left(\mathbf{R}_{g}^{-1} \mathbf{x}\right)
$$


Following the development of Equation 6 we have

$$
\begin{aligned}
P_{i}^{j k} w\left(\mathbf{R}_{h}^{-1} \mathbf{x}\right) & =\frac{d_{i}}{n} \sum_{g \in \mathcal{G}} \rho_{i}^{j k}\left(\sigma_{g}\right) w\left(\mathbf{R}_{g}^{-1}\left(\mathbf{R}_{h}^{-1} \mathbf{x}\right)\right) \\
& =\frac{d_{i}}{n} \sum_{g \in \mathcal{G}} \sum_{s=1}^{d_{i}} \overbrace{\rho_{i}^{j s}\left(\sigma_{h}^{-1} \sigma_{h}\right)}^{=\delta_{j s}} \rho_{i}^{s k}\left(\sigma_{g}\right) w\left(\left(\mathbf{R}_{h} \mathbf{R}_{g}\right)^{-1} \mathbf{x}\right) \\
& =\frac{d_{i}}{n} \sum_{g \in \mathcal{G}} \sum_{r=1}^{d_{i}} \sum_{s=1}^{d_{i}} \rho_{i}^{j r}\left(\sigma_{h}^{-1}\right) \rho_{i}^{r s}\left(\sigma_{h}\right) \rho_{i}^{s k}\left(\sigma_{g}\right) w\left(\left(\mathbf{R}_{h} \mathbf{R}_{g}\right)^{-1} \mathbf{x}\right) \\
& =\frac{d_{i}}{n} \sum_{g \in \mathcal{G}} \sum_{r=1}^{d_{i}} \rho_{i}^{r j}\left(\sigma_{h}\right) \rho_{i}^{r k}\left(\sigma_{h} \sigma_{g}\right) w\left(\left(\mathbf{R}_{h} \mathbf{R}_{g}\right)^{-1} \mathbf{x}\right) \\
& =\sum_{r=1}^{d_{i}} \rho_{i}^{r j}\left(\sigma_{h}\right) \frac{d_{i}}{n} \sum_{g \in \mathcal{G}} \rho_{i}^{r k}\left(\sigma_{h} \sigma_{g}\right) w\left(\left(\mathbf{R}_{h} \mathbf{R}_{g}\right)^{-1} \mathbf{x}\right) \\
& =\sum_{r=1}^{d_{i}} \rho_{i}^{r j}\left(\sigma_{h}\right) P_{i}^{r k} w(\mathbf{x})
\end{aligned}
$$

Using the orthogonality of the irreps and manipulating the above also gives

$$
\begin{aligned}
P_{i}^{j k} w\left(\mathbf{R}_{h} \mathbf{x}\right) & =\sum_{r=1}^{d_{i}} \rho_{i}^{r j}\left(\sigma_{h}^{-1}\right) P_{i}^{r k} w(\mathbf{x}) \\
& =\sum_{r=1}^{d_{i}}\left(\left[\boldsymbol{\rho}_{i}\left(\sigma_{h}\right)\right]^{T}\right)^{r j} P_{i}^{r k} w(\mathbf{x}) \\
& =\sum_{r=1}^{d_{i}} \rho_{i}^{j r}\left(\sigma_{h}\right) P_{i}^{r k} w(\mathbf{x})
\end{aligned}
$$

which can be recast as matrix equation, cf. Equation 7

$$
\mathbf{P}_{i}^{k} w\left(\mathbf{R}_{h} \mathbf{x}\right)=\boldsymbol{\rho}_{i}\left(\sigma_{h}\right) \mathbf{P}_{i}^{k} w(\mathbf{x})
$$

where $\mathbf{P}_{i}^{k} w=\left[\begin{array}{llll}P_{i}^{1 k} w & P_{i}^{2 k} w & \cdots & P_{i}^{d_{i} k} w\end{array}\right]^{T}$ is the so-called " $i-k$ family" which plays an important role in the subsequent analysis.

And following the development of Equation 8 we find

$$
\begin{aligned}
P_{i}^{j k} P_{r}^{s t} w(\mathbf{x}) & =\frac{d_{i}}{n} \sum_{g \in \mathcal{G}} \rho_{i}^{j k}\left(\sigma_{g}\right) P_{r}^{s t} w\left(\mathbf{R}_{g}^{-1} \mathbf{x}\right) \\
& =\frac{d_{i}}{n} \sum_{g \in \mathcal{G}} \rho_{i}^{j k}\left(\sigma_{g}\right) \sum_{u=1}^{d_{r}} \rho_{r}^{u s}\left(\sigma_{g}\right) P_{r}^{u t} w(\mathbf{x}) \\
& =\frac{d_{i}}{n} \sum_{u=1}^{d_{r}} P_{r}^{u t} w(\mathbf{x}) \sum_{g \in \mathcal{G}} \rho_{i}^{j k}\left(\sigma_{g}\right) \rho_{r}^{u s}\left(\sigma_{g}\right) \\
& =\frac{d_{i}}{n} \sum_{u=1}^{d_{r}} P_{r}^{u t} w(\mathbf{x}) \delta_{i r} \delta_{j u} \delta_{k s} \frac{n}{d_{i}} \\
& =\delta_{i r} \delta_{k s} P_{r}^{j t} w(\mathbf{x})
\end{aligned}
$$

where we again utilized the row-wise orthogonality of the irreps. For $D_{3}$, e.g. $P_{1} P_{2} w(\mathbf{x})=0, P_{1} P_{3}^{12} w(\mathbf{x})=0$ and $P_{3}^{21} P_{3}^{22} w(\mathbf{x})=0$ along with $P_{1} P_{1} w(\mathbf{x})=P_{1} w(\mathbf{x})$ and $P_{3}^{11} P_{3}^{12} w(\mathbf{x})=P_{3}^{12} w(\mathbf{x})$. As seen here and noted 
in Section 1.2, the projections associated with irreps $\boldsymbol{\rho}_{i}$ of order $d_{i}>1$ are not mutually orthogonal to one another; they are, however orthogonal to the projections associated with any other irrep $\boldsymbol{\rho}_{r}$ such that $r \neq i$.

Slightly different than Equation 9, for the nonabelian we have

$$
w(\mathbf{x})=\sum_{i=1}^{n_{\rho}} \sum_{j=1}^{d_{i}} P_{i}^{j j} w(\mathbf{x})
$$

where $n_{\rho}$ denotes the number of irreps, here $n_{\rho}=3$. So again, we are able to break $\mathcal{H}$ into complementary subspaces, i.e. $\mathcal{H}(\Omega)=\bigoplus_{i=1}^{n_{\rho}} \bigoplus_{j=1}^{d_{i}} P_{i}^{j j} \mathcal{H}(\Omega)$. Note that the "mixed terms," e.g. $P_{3}^{12}$, do not appear in the sum of Equation 25. However, as soon seen, they are of vital importance. The proof of the above follows from Equation 10, i.e.

$$
\begin{aligned}
\sum_{i=1}^{n_{\rho}} \sum_{j=1}^{d_{i}} P_{i}^{j j} w(\mathbf{x}) & =\sum_{i=1}^{n_{\rho}} \sum_{j=1}^{d_{i}} \frac{d_{i}}{n} \sum_{g} \rho_{i}^{j j}\left(\sigma_{g}\right) w\left(\mathbf{R}_{g}^{-1} \mathbf{x}\right) \\
& =\sum_{g} w\left(\mathbf{R}_{g}^{-1} \mathbf{x}\right) \sum_{i=1}^{n_{\rho}} \sum_{j=1}^{d_{i}} \frac{d_{i}}{n} \rho_{i}^{j j}\left(\sigma_{g}\right) \\
& =\sum_{g} w\left(\mathbf{R}_{g}^{-1} \mathbf{x}\right) \sum_{i=1}^{n_{\rho}} \sum_{j=1}^{d_{i}} \sum_{k=1}^{d_{i}} \overbrace{\rho_{i}^{k j}\left(\sigma_{1}\right)}^{\delta_{j k}} \frac{d_{i}}{n} \rho_{i}^{k j}\left(\sigma_{g}\right) \\
& =\sum_{g} w\left(\mathbf{R}_{g}^{-1} \mathbf{x}\right) \delta_{1 g} \\
& =w\left(\mathbf{R}_{1}^{-1} \mathbf{x}\right) \\
& =w(\mathbf{x})
\end{aligned}
$$

where the $\sum_{i=1}^{n_{\rho}} \sum_{j=1}^{d_{i}} \sum_{k=1}^{d_{i}} \rho_{i}^{k j}\left(\sigma_{1}\right) \frac{d_{i}}{n} \rho_{i}^{k j}\left(\sigma_{g}\right)=\delta_{1 g}$ result again follows from properties 3 and 4 of the irrep table. Here we define the matrix $\rho$ as described in the discussion surrounding property 3 and use properties 3 and 4 to obtain $\boldsymbol{\rho} \boldsymbol{\rho}^{T}=n \operatorname{diag}\left(\left(1 / d_{1}\right) \mathbf{I},\left(1 / d_{2}\right) \mathbf{I}, \ldots,\left(1 / d_{n}\right) \mathbf{I}\right)$. This implies det $\boldsymbol{\rho} \neq 0$ and $\boldsymbol{\rho}^{-1}=\boldsymbol{\rho}^{T}(1 / n) \operatorname{diag}\left(d_{1} \mathbf{I}, d_{2} \mathbf{I}, \ldots, d_{n} \mathbf{I}\right)$. The fact that $\boldsymbol{\rho}^{-1} \boldsymbol{\rho}=\mathbf{I}$ (and the discussion surrounding property 3 regarding the form of $\boldsymbol{\rho})$ gives the result $\sum_{i=1}^{n_{\rho}} \sum_{j=1}^{d_{i}} \sum_{k=1}^{d_{i}} \rho_{i}^{k j}\left(\sigma_{1}\right) \frac{d_{i}}{n} \rho_{i}^{k j}\left(\sigma_{g}\right)=\delta_{1 g}$. Slightly different from the abelian case, when $d_{i}>1$ the $P_{i}^{j j} \mathcal{H}(\Omega)$ are not invariant with respect to the group actions. However, the sum $\bigoplus_{j=1}^{d_{i}} P_{i}^{j j} \mathcal{H}(\Omega)$ is invariant.

Following the developments of $11-13$ and $22-24$ we note that

$$
\begin{aligned}
\nabla P_{i}^{j k} w\left(\mathbf{R}_{h}^{-1} \mathbf{x}\right) & =\mathbf{R}_{h}^{-1} \sum_{r=1}^{d_{i}} \rho_{i}^{r j}\left(\sigma_{h}\right) \nabla P_{i}^{r k} w(\mathbf{x}) \\
\nabla P_{i}^{j k} w\left(\mathbf{R}_{h} \mathbf{x}\right) & =\mathbf{R}_{h} \sum_{r=1}^{d_{i}} \rho_{i}^{j r}\left(\sigma_{h}\right) \nabla P_{i}^{r k} w(\mathbf{x})
\end{aligned}
$$

as well as

$$
\begin{aligned}
P_{i}^{j k} \chi(\mathbf{x}) & =\frac{d_{i}}{n} \sum_{g \in \mathcal{G}} \rho_{i}^{j k}\left(\sigma_{g}\right) \mathbf{R}_{g} \chi\left(\mathbf{R}_{g}^{-1} \mathbf{x}\right) \\
P_{i}^{j k} \chi\left(\mathbf{R}_{h}^{-1} \mathbf{x}\right) & =\mathbf{R}_{h}^{-1} \sum_{r=1}^{d_{i}} \rho_{i}^{j r}\left(\sigma_{h}\right) P_{i}^{r k} \chi(\mathbf{x}) \\
P_{i}^{j k} \chi\left(\mathbf{R}_{h} \mathbf{x}\right) & =\mathbf{R}_{h} \sum_{r=1}^{d_{i}} \rho_{i}^{j r}\left(\sigma_{h}\right) P_{i}^{r k} \chi(\mathbf{x})
\end{aligned}
$$

Before proceeding it is worth revisiting the solution to the problem $\mathbf{A} \mathbf{x}=\mathbf{b}$ where $\mathbf{A}$ is still $n \times n$ but it now contains one or more repeated eigenvalues and is such that it is not possible to find $n$ linearly 
independent eigenvectors. In such cases the Jordan canonical form can be used to almost diagonalize the system. So while we do not obtain the simplification discussed in section 2.1, we still obtain a block diagonal structure which is easily decomposed. For our $D_{3}$ analogy $\mathbf{A}$ is $6 \times 6$ with 2 distinct eigenvalues and two pairs of repeated eigenvalues; its "canonical form" resembles

$$
\hat{\mathbf{A}}_{6 \times 6}=\left[\begin{array}{cccc}
\hat{A}_{1 \times 1}^{1} & & & \\
& \hat{A}_{1 \times 1}^{2} & & \\
& & \hat{\mathbf{A}}_{2 \times 2}^{3} & \\
& & & \hat{\mathbf{A}}_{2 \times 2}^{4}
\end{array}\right]
$$

Whereas for the abelian order $n=4 D_{2}$ analogy $\mathbf{A}$ is $4 \times 4$ with 4 distinct eigenvalues; its "canonical form" resembles $\hat{\mathbf{A}}_{4 \times 4}=\operatorname{diag}\left(\hat{A}_{1 \times 1}^{1}, \hat{A}_{1 \times 1}^{2}, \hat{A}_{1 \times 1}^{3}, \hat{A}_{1 \times 1}^{4}\right)$. So on a $D_{3}$ domain we solve 2 one-dimensional problems and 2 two-dimensional problems whereas on a $D_{2}$ domain we solve 4 one-dimensional problems.

We return to the integral Equation 4 and follow the derivation of Equation 14. Here we substitute $v_{i}^{j j}=P_{i}^{j j} v$ into Equation 4 which gives

$$
\begin{aligned}
& \int_{\Omega} \nabla v_{i}^{j j}(\mathbf{x}) \cdot \mathbf{k}(\mathbf{x}) \nabla w(\mathbf{x}) d v=\int_{\Omega}\left(\nabla v_{i}^{j j}(\mathbf{x}) \cdot \mathbf{k}(\mathbf{x}) \boldsymbol{\chi}(\mathbf{x})+v_{i}^{j j}(\mathbf{x}) f(\mathbf{x})\right) d v+ \\
& \int_{\partial \Omega^{n}} v_{i}^{j j}(\mathbf{x}) q^{p}(\mathbf{x}) d a \\
& \sum_{g \in \mathcal{G}} \int_{\mathbf{R}_{g}^{-1}(C)} \nabla v_{i}^{j j}(\mathbf{x}) \cdot \mathbf{k}(\mathbf{x}) \nabla w(\mathbf{x}) d v \quad=\quad \sum_{g \in \mathcal{G}} \int_{\mathbf{R}_{g}^{-1}(C)}\left(\nabla v_{i}^{j j}(\mathbf{x}) \cdot \mathbf{k}(\mathbf{x}) \boldsymbol{\chi}(\mathbf{x})+v_{i}^{j j}(\mathbf{x}) f(\mathbf{x})\right) d v+ \\
& \sum_{g \in \mathcal{G}} \int_{\mathbf{R}_{g}^{-1}\left(\partial C^{n}\right)} v_{i}^{j j}(\mathbf{x}) q^{p}(\mathbf{x}) d a \\
& \sum_{g \in \mathcal{G}} \int_{C} \nabla v_{i}^{j j}\left(\mathbf{R}_{g}^{-1} \mathbf{x}\right) \cdot \mathbf{k}\left(\mathbf{R}_{g}^{-1} \mathbf{x}\right) \nabla w\left(\mathbf{R}_{g}^{-1} \mathbf{x}\right) d v \quad=\sum_{g \in \mathcal{G}} \int_{C}\left(\nabla v_{i}^{j j}\left(\mathbf{R}_{g}^{-1} \mathbf{x}\right) \cdot \mathbf{k}\left(\mathbf{R}_{g}^{-1} \mathbf{x}\right) \chi\left(\mathbf{R}_{g}^{-1} \mathbf{x}\right)+\right. \\
& \left.v_{i}^{j j}\left(\mathbf{R}_{g}^{-1} \mathbf{x}\right) f\left(\mathbf{R}_{g}^{-1} \mathbf{x}\right)\right) d v+ \\
& \sum_{g \in \mathcal{G}} \int_{\partial C^{n}} v_{i}^{j j}\left(\mathbf{R}_{g}^{-1} \mathbf{x}\right) q^{p}\left(\mathbf{R}_{g}^{-1} \mathbf{x}\right) d a \\
& \sum_{g \in \mathcal{G}} \sum_{l=1}^{d_{i}} \int_{C} \rho_{i}^{l j}\left(\sigma_{g}\right) \mathbf{R}_{g}^{-1} \nabla v_{i}^{l j}(\mathbf{x}) \cdot \mathbf{R}_{g}^{-1} \mathbf{k}(\mathbf{x}) \mathbf{R}_{g} \nabla w\left(\mathbf{R}_{g}^{-1} \mathbf{x}\right) d v=\sum_{g \in \mathcal{G}} \sum_{l=1}^{d_{i}} \int_{C}\left(\rho_{i}^{l j}\left(\sigma_{g}\right) \mathbf{R}_{g}^{-1} \nabla v_{i}^{l j}(\mathbf{x}) \cdot \mathbf{R}_{g}^{-1} \mathbf{k}(\mathbf{x}) \mathbf{R}_{g} \chi\left(\mathbf{R}_{g}^{-1} \mathbf{x}\right)+\right. \\
& \left.\rho_{i}^{l j}\left(\sigma_{g}\right) v_{i}^{l j}(\mathbf{x}) f\left(\mathbf{R}_{g}^{-1} \mathbf{x}\right)\right) d v+ \\
& \sum_{g \in \mathcal{G}} \sum_{l=1}^{d_{i}} \int_{\partial C^{n}} \rho_{i}^{l j}\left(\sigma_{g}\right) v_{i}^{l j}(\mathbf{x}) q^{p}\left(\mathbf{R}_{g}^{-1} \mathbf{x}\right) d a \\
& \sum_{g \in \mathcal{G}} \sum_{l=1}^{d_{i}} \int_{C} \nabla v_{i}^{l j}(\mathbf{x}) \cdot \mathbf{k}(\mathbf{x}) \rho_{i}^{l j}\left(\sigma_{g}\right) \mathbf{R}_{g} \nabla w\left(\mathbf{R}_{g}^{-1} \mathbf{x}\right) d v \quad=\sum_{g \in \mathcal{G}} \sum_{l=1}^{d_{i}} \int_{C}\left(\nabla v_{i}^{l j}(\mathbf{x}) \cdot \mathbf{k}(\mathbf{x}) \rho_{i}^{l j}\left(\sigma_{g}\right) \mathbf{R}_{g} \boldsymbol{\chi}\left(\mathbf{R}_{g}^{-1} \mathbf{x}\right)+\right. \\
& \left.v_{i}^{l j}(\mathbf{x}) \rho_{i}^{l j}\left(\sigma_{g}\right) f\left(\mathbf{R}_{g}^{-1} \mathbf{x}\right)\right) d v+ \\
& \sum_{g \in \mathcal{G}} \sum_{l=1}^{d_{i}} \int_{\partial C^{n}} v_{i}^{l j}(\mathbf{x}) \rho_{i}^{l j}\left(\sigma_{g}\right) q^{p}\left(\mathbf{R}_{g}^{-1} \mathbf{x}\right) d a \\
& \frac{n}{d_{i}} \sum_{l=1}^{d_{i}} \int_{C} \nabla v_{i}^{l j}(\mathbf{x}) \cdot \mathbf{k}(\mathbf{x}) \nabla w_{i}^{l j}(\mathbf{x}) d v=\frac{n}{d_{i}} \sum_{l=1}^{d_{i}} \int_{C}\left(\nabla v_{i}^{l j}(\mathbf{x}) \cdot \mathbf{k}(\mathbf{x}) \boldsymbol{\chi}_{i}^{l j}(\mathbf{x})+\right. \\
& \left.v_{i}^{l j}(\mathbf{x}) f_{i}^{l j}(\mathbf{x})\right) d v+ \\
& \frac{n}{d_{i}} \sum_{l=1}^{d_{i}} \int_{\partial C^{n}} v_{i}^{l j}(\mathbf{x}) q_{i}^{p^{l j}}(\mathbf{x}) d a
\end{aligned}
$$

where here and henceforth it is understood that, e.g. $w_{i}^{l j}=P_{i}^{l j} w$. To obtain the above we used the summation of integration, change-of-variable theorem, Equations $21-22$ and the domain symmetry of $\mathbf{k}$.

Now we follow the development of Equation 15 wherein we now use Equation 25 to express $v=$ $\sum_{i=1}^{n_{\rho}} \sum_{j=1}^{d_{i}} v_{i}^{j j}$ and substitute into Equation 4. Each $v_{i}^{j j}$ renders an integral expression which only contains $w_{i}^{l j}$ for $l=1,2, \ldots, d_{i}$. We evaluate the response $w_{i}^{l j}$ by setting $v_{i}^{m j}=0$ for $m \neq l$. In light of this the boundary value problem of Equation 4 defined on $\Omega$ is now converted into $n=\sum_{i=1}^{n_{\rho}} d_{i}^{2}$ problems defined on 
$C$ which read: find the responses $w_{i}^{l j} \in \mathcal{H}\left(C, w_{i}^{p^{l j}}\right)$ such that

$$
\int_{C} \nabla v_{i}^{l j} \cdot \mathbf{k} \nabla w_{i}^{l j} d v=\int_{C}\left(\nabla v_{i}^{l j} \cdot \mathbf{k} \chi_{i}^{l j}+v_{i}^{l j} f_{i}^{l j}\right) d v+\int_{\partial C^{n}} v_{i}^{l j} q_{i}^{p^{l j}} d a
$$

for all $v_{i}^{l j} \in \mathcal{H}(C, 0)$. The obvious benefit these problems offer is their replacement of one problem defined over the large domain $\Omega$ by $n$ problems defined over the smaller symmetry cell $C$.

As before, the above result is not satisfactory because we have not incorporated the boundary conditions on the cut surfaces of $C$, i.e. the boundary $\Sigma=\partial C \backslash\left(\partial C^{d} \cup \partial C^{n}\right)$, cf. Figure 4. For example, referring to Figure 4, a point $\mathbf{x} \in C$ is on $\Sigma_{y 2} \subset \Sigma$ if $\mathbf{x}=\mathbf{R}_{y 2} \mathbf{x}$; it is on $\Sigma_{y} \subset \Sigma$ if $\mathbf{x}=\mathbf{R}_{y} \mathbf{x}$. Again, we allow neither jumps in the response nor in the flux throughout the domain, and thus the $w_{i}^{l j}$ for $i=1, \ldots, n_{\rho}$ and $l, j=1, \ldots, d_{i}$ at each of these points must satisfy

$$
\begin{aligned}
\llbracket w_{i}^{l j}(\mathbf{x}) \rrbracket & =0 \\
\llbracket \mathbf{k}(\mathbf{x})\left(\nabla w_{i}^{l j}(\mathbf{x})-\chi_{i}^{l j}(\mathbf{x})\right) \rrbracket \cdot \mathbf{n}(\mathbf{x}) & =0
\end{aligned}
$$

Considering a general cut boundary point $\mathbf{x}=\mathbf{R}_{g} \mathbf{x} \in \Sigma_{g}$ and substituting Equation 23 reduces the first jump condition to

$$
\begin{aligned}
w_{i}^{l j}(\mathbf{x}) & =w_{i}^{l j}\left(\mathbf{R}_{g} \mathbf{x}\right) \\
& =\sum_{r=1}^{d_{i}} \rho_{i}^{l r}\left(\sigma_{g}\right) w_{i}^{r j}(\mathbf{x})
\end{aligned}
$$

which can alternately be expressed as

$$
\frac{1}{2}\left(\mathbf{I}-\boldsymbol{\rho}_{i}\left(\sigma_{g}\right)\right) \mathbf{w}_{i}^{l}(\mathbf{x})=\mathbf{0}
$$

where $\mathbf{w}_{i}^{l}=\left[\begin{array}{llll}w_{i}^{1 l} & w_{i}^{2 l} & \ldots & w_{i}^{d_{i} l}\end{array}\right]^{T}$ is the $i-l$ family of functions and $\mathbf{I}$ is the $d_{i} \times d_{i}$ identity. These $l=1,2, \ldots, d_{i}$ "constraint equations" must be satisfied and hence we see that the problems of Equation 29 involving the family $\mathbf{w}_{i}^{l}=\left[\begin{array}{llll}w_{i}^{1 l} & w_{i}^{2 l} & \ldots & w_{i}^{d_{i} l}\end{array}\right]^{T}$ are coupled. The role the mixed terms, e.g. $w_{3}^{21}$ is illuminated here. Indeed, they are needed to compute $w_{i}^{l l}$ which is used in Equation 25 to compute $w$. Also of note here is the form of Equation 31 wherein we recognize that $\left(1, \mathbf{w}_{i}^{l}(\mathbf{x})\right)$ is an eigenpair of $\boldsymbol{\rho}_{i}$, assuming $\mathbf{w}_{i}^{l}(\mathbf{x})$ is real and nonzero. ${ }^{7}$ Thusly, less than $d_{i}$ independent constraint equations are generated at each point $\mathbf{x} \in \Sigma_{g}$ from Equation 31, depending on the multiplicity of the $\lambda=1$ eigenvalue.

As in the abelian case, we need not concern ourselves with the flux jump condition as the resulting homogeneous natural boundary conditions are satisfied defacto in the weak formulation. Nonetheless, for completeness we derive them by substituting Equations 27 and 28 to reduce the second jump Equation 30 to

$$
\begin{aligned}
\mathbf{k}(\mathbf{x})\left(\nabla w_{i}^{l j}(\mathbf{x})-\chi_{i}^{l j}(\mathbf{x})\right) \cdot \mathbf{n}(\mathbf{x}) & =\mathbf{k}\left(\mathbf{R}_{g} \mathbf{x}\right)\left(\nabla w_{i}^{l j}\left(\mathbf{R}_{g} \mathbf{x}\right)-\chi_{i}^{l j}\left(\mathbf{R}_{g} \mathbf{x}\right)\right) \cdot \mathbf{n}(\mathbf{x}) \\
& =\mathbf{R}_{g} \mathbf{k}(\mathbf{x}) \mathbf{R}_{g}^{-1} \sum_{r=1}^{d_{i}} \rho_{i}^{l r}\left(\sigma_{g}\right) \mathbf{R}_{g}\left(\nabla w_{i}^{r j}(\mathbf{x})-\chi_{i}^{r j}(\mathbf{x})\right) \cdot \mathbf{n}(\mathbf{x}) \\
& =\mathbf{k}(\mathbf{x}) \sum_{r=1}^{d_{i}} \rho_{i}^{l r}\left(\sigma_{g}\right)\left(\nabla w_{i}^{r j}(\mathbf{x})-\chi_{i}^{r j}(\mathbf{x})\right) \cdot \mathbf{R}_{g}^{T} \mathbf{n}(\mathbf{x}) \\
& =-\mathbf{k}(\mathbf{x}) \sum_{r=1}^{d_{i}} \rho_{i}^{l r}\left(\sigma_{g}\right)\left(\nabla w_{i}^{r j}(\mathbf{x})-\chi_{i}^{r j}(\mathbf{x})\right) \cdot \mathbf{n}(\mathbf{x})
\end{aligned}
$$

where we use the fact that $\mathbf{R}_{g}^{T} \mathbf{n}(\mathbf{x})=-\mathbf{n}(\mathbf{x})$ for $\mathbf{x} \in \Sigma_{g}$. Alternatively, the above can be expressed as

$$
\frac{1}{2}\left(\mathbf{I}+\boldsymbol{\rho}_{i}\left(\sigma_{g}\right)\right) \mathbf{q}_{i}^{n^{l}}(\mathbf{x})=\mathbf{0}
$$

\footnotetext{
${ }^{7}$ The real eigenvalues of an orthogonal matrix equal \pm 1 .
} 
where $\mathbf{q}_{i}^{n^{l}}=\left[\begin{array}{llll}q_{i}^{1 l} & q_{i}^{2 l} & \ldots & q_{i}^{d_{i} l}\end{array}\right]^{T}=\left[\begin{array}{lllll}\mathbf{k}\left(\nabla w_{i}^{1 l}-\chi_{i}^{1 l}\right) \cdot \mathbf{n} & \mathbf{k}\left(\nabla w_{i}^{2 l}-\chi_{i}^{2 l}\right) \cdot \mathbf{n} & \ldots & \mathbf{k}\left(\nabla w_{i}^{d_{i} l}-\chi_{i}^{d_{i} l}\right) \cdot \mathbf{n}\end{array}\right]^{T}$ consolidates the $i-l$ family of fluxes. In regard to the discussion after Equation 18, here we see that the $1 / 2\left(\mathbf{I}-\boldsymbol{\rho}_{i}\left(\sigma_{g}\right)\right)$ in Equation 31 and the $1 / 2\left(\mathbf{I}+\boldsymbol{\rho}_{i}\left(\sigma_{g}\right)\right)$ in Equation 32 are complementary projections. And as per the conversation after Equation 31, we see that $\left(-1, \mathbf{q}_{i}^{n^{l}}(\mathbf{x})\right)$ is an eigenpair of $\boldsymbol{\rho}_{i}$, assuming $\mathbf{q}_{i}^{n^{l}}(\mathbf{x})$ is real and nonzero; hence, less that $d_{i}$ independent flux constraint equations are generated from Equation 32.

Once the responses $w_{i}^{j k}$ on $C$ are computed they are used to evaluate $w_{i}$ throughout $\Omega$ via Equation 23 and summed via Equation 25 to evaluate $w$, i.e.

$$
w\left(\mathbf{R}_{g} \mathbf{x}\right)=\sum_{i=1}^{n_{\rho}} \sum_{j=1}^{d_{i}} w_{i}^{j j}\left(\mathbf{R}_{g} \mathbf{x}\right)=\sum_{i=1}^{n_{\rho}} \sum_{j=1}^{d_{i}} \sum_{k=1}^{d_{i}} \rho_{i}^{j k}\left(\sigma_{g}\right) w_{i}^{k j}(\mathbf{x}),
$$

where $\mathbf{x} \in C$. The role of the mixed terms, e.g. $w_{3}^{21}$, is again seen.

For our $D_{3}$ example we have $d_{1}=1, d_{2}=1$ and $d_{3}=2$ and thusly there is no coupling for the $w_{1}$ and $w_{2}$ problems.

1. Concerning the $w_{1}$ problem for both $\mathbf{x} \in \Sigma_{y}$ and $\mathbf{x} \in \Sigma_{y 2}$ Equation 31 with $g=y, y 2$ gives $(1-$ $\left.\rho_{1}\left(\sigma_{g}\right)\right) w_{1}=0$ which is necessarily satisfied. Again for both surfaces Equation 32 yields $\left(1+\rho_{1}\left(\sigma_{g}\right)\right) q_{1}=$ $2 q_{1}$ which implies $q_{1}=0$.

2. In regard to the $w_{2}$ problem for both $\mathbf{x} \in \Sigma_{y}$ and $\mathbf{x} \in \Sigma_{y 2}$ Equation 31 with $g=y, y 2$ gives (1$\left.\rho_{2}\left(\sigma_{g}\right)\right) w_{2}=2 w_{2}$ which implies $w_{2}=0$. Again for both surfaces Equation 32 yields $\left(1+\rho_{2}\left(\sigma_{g}\right)\right) q_{2}=0$ which is automatically satisfied.

3. Now we consider the coupled $\mathbf{w}_{3}^{1}=\left[\begin{array}{ll}w_{3}^{11} & w_{3}^{21}\end{array}\right]^{T}$ problem. Equation 31 for $\mathbf{x} \in \Sigma_{y}$ implies $1 / 2(\mathbf{I}-$ $\left.\boldsymbol{\rho}_{3}\left(\sigma_{y}\right)\right) \mathbf{w}_{3}^{1}=\left[\begin{array}{ll}0 & 0 \\ 0 & 1\end{array}\right] \mathbf{w}_{3}^{1}=\mathbf{0}$ or $w_{3}^{21}=0$ on $\Sigma_{y}$. Equation 32 meanwhile yields $1 / 2\left(\mathbf{I}+\boldsymbol{\rho}_{3}\left(\sigma_{y}\right)\right) \mathbf{q}_{3}^{n^{1}}=$ $\left[\begin{array}{ll}1 & 0 \\ 0 & 0\end{array}\right] \mathbf{q}_{3}^{n^{1}}=\mathbf{0}$ which implies $q_{3}^{n^{11}}=0$.

For $\mathbf{x} \in \Sigma_{y 2}$ we have $1 / 2\left(\mathbf{I}-\boldsymbol{\rho}_{3}\left(\sigma_{y 2}\right)\right) \mathbf{w}_{3}^{1}=1 / 4\left[\begin{array}{cc}3 & \sqrt{3} \\ \sqrt{3} & 1\end{array}\right] \mathbf{w}_{3}^{1}=\mathbf{0}$ which yields the single constraint equation $\sqrt{3} w_{3}^{11}+w_{3}^{21}=0$ from both rows. Equation 32 with $l=1$ yields $1 / 2\left(\mathbf{I}+\boldsymbol{\rho}_{3}\left(\sigma_{y 2}\right)\right) \mathbf{q}_{3}^{n^{1}}=$ $1 / 4\left[\begin{array}{cc}1 & -\sqrt{3} \\ -\sqrt{3} & 3\end{array}\right] \mathbf{q}_{3}^{n^{1}}=\mathbf{0}$ which implies $q_{3}^{n^{11}}-\sqrt{3} q_{3}^{n^{21}}=0$ from both rows.

As seen here, $d_{i}-1=2-1=1$ independent constraint equations relating $w_{3}^{11}$ and $w_{3}^{21}$ are generated on each of the $\Sigma$ surfaces.

4. Finally, we consider the coupled $\mathbf{w}_{3}^{2}=\left[\begin{array}{ll}w_{3}^{12} & w_{3}^{22}\end{array}\right]^{T}$ problem. These constraints parrot those of the coupled $\mathbf{w}_{3}^{1}=\left[\begin{array}{ll}w_{3}^{11} & w_{3}^{21}\end{array}\right]^{T}$ problem. Indeed, upon inspection of Equations 31 and 32 we see that the only difference between the two problems is that the second " 1 " superscript on $\mathbf{w}$ and $\mathbf{q}$ is replaced by a " 2 ".

Summarizing, for the $D_{3}$ symmetric domain we solve two uncoupled boundary value problems on $C$. For $w_{1}$ we satisfy

$$
\begin{aligned}
\operatorname{div}\left(\mathbf{k}\left(\nabla w_{1}-\chi_{1}\right)+f_{1}\right. & =0 & & \text { on } C \\
w_{1} & =w_{1}^{p} & & \text { on } \partial C^{d} \\
q_{1} & =q_{1}^{p} & & \text { on } \partial C^{n} \\
q_{1} & =0 & & \text { on } \Sigma_{y} \cup \Sigma_{y 2}
\end{aligned}
$$

and for $w_{2}$ we satisfy

$$
\begin{aligned}
\operatorname{div}\left(\mathbf{k}\left(\nabla w_{2}-\chi_{2}\right)+f_{2}\right. & =0 & & \text { on } C \\
w_{2} & =w_{2}^{p} & & \text { on } \partial C^{d} \\
q_{2} & =q_{2}^{p} & & \text { on } \partial C^{n} \\
w_{2} & =0 & & \text { on } \Sigma_{y} \cup \Sigma_{y 2}
\end{aligned}
$$


We also solve 2 sets of coupled problems ${ }^{8}$ for the pairs $\left(w_{3}^{11}, w_{3}^{21}\right)$ and $\left(w_{3}^{12}, w_{3}^{22}\right)$ such that

$$
\begin{aligned}
\operatorname{div}\left(\mathbf{k}\left(\nabla w_{3}^{r l}-\chi_{3}^{r l}\right)+f_{3}^{r l}\right. & =0 & & \text { in } C \\
w_{3}^{r l} & =w_{3}^{p^{r l}} & & \text { on } \partial C^{d} \\
q_{3}^{r l} & =q_{3}^{p^{r l}} & & \text { on } \partial C^{n} \\
w_{3}^{2 l} & =0 & & \text { on } \Sigma_{y} \\
q_{3}^{1 l} & =0 & & \text { on } \Sigma_{y} \\
\sqrt{3} w_{3}^{1 l}+w_{3}^{2 l} & =0 & & \text { on } \Sigma_{y 2} \\
q_{3}^{1 l}-\sqrt{3} q_{3}^{2 l} & =0 & & \text { on } \Sigma_{y 2}
\end{aligned}
$$

The complementarity of boundary conditions amongst the family $\mathbf{w}_{3}^{l}=\left[\begin{array}{ll}w_{3}^{1 l} & w_{3}^{2 l}\end{array}\right]^{T}$ and its associated flux $\mathbf{q}_{3}^{n^{l}}=\left[\begin{array}{ll}q_{3}^{1 l} & q_{3}^{2 l}\end{array}\right]^{T}$ on the cut surfaces are readily seen on $\Sigma_{y}$. As a mechanical analogy, the $w_{3}^{2 l}=0$ and $q_{3}^{1 l}=0$ boundary condition on $\Sigma_{y}$ is one of zero displacement in the $\mathbf{e}_{2}$ direction and zero traction in the $\mathbf{e}_{1}$ direction, i.e. a sliding boundary condition. A similar relation is seen on $\Sigma_{2 y}$ where the displacement vector that satisfies $\sqrt{3} w_{3}^{1 l}+w_{3}^{2 l}=0$ slides along $\Sigma_{2 y}$, while the traction vector which satisfies $q_{3}^{1 l}-\sqrt{3} q_{3}^{2 l}=0$ is perpendicular to $\Sigma_{2 y}$. These null Neumann flux conditions on the cut surfaces are necessarily satisfied in the weak formulation. The constraints on the $w_{3}^{i j}$, on the other hand, are not.

To satisfy the nontrivial constraints we use Lagrange multipliers so that the weak form of Equation 34, reads: Find $w_{3}^{1 l} \in \mathcal{H}\left(C, w_{3}^{p^{1 l}}, 0\right), w_{3}^{2 l} \in \mathcal{H}\left(C, w_{3}^{p^{2 l}}\right)$ and $\lambda \in \mathcal{L}(C)$ such that

$$
\begin{aligned}
& \int_{C} \nabla v_{3}^{1 l} \cdot \mathbf{k} \nabla w_{3}^{1 l} d a+\int_{C} \nabla v_{3}^{2 l} \cdot \mathbf{k} \nabla w_{3}^{2 l} d a+\int_{\Sigma_{y 2}} \beta\left(\sqrt{3} w_{3}^{1 l}+w_{3}^{2 l}\right) d l+\int_{\Sigma_{y 2}} \lambda\left(\sqrt{3} v_{3}^{1 l}+v_{3}^{2 l}\right) d l= \\
& {\left[\int_{C}\left(\nabla v_{3}^{1 l} \cdot \mathbf{k} \chi_{3}^{1 l}+v_{3}^{1 l} f_{3}^{1 l}\right) d a+\int_{\partial C^{n}} v_{3}^{1 l} q_{3}^{p^{1 l}} d l\right]+\left[\int_{C}\left(\nabla v_{3}^{2 l} \cdot \mathbf{k} \chi_{3}^{2 l}+v_{3}^{2 l} f_{3}^{2 l}\right) d a+\int_{\partial C^{n}} v_{3}^{2 l} q_{3}^{p^{2 l}} d l\right]}
\end{aligned}
$$

for all $v_{3}^{1 l} \in \mathcal{H}(C, 0,0), v_{3}^{2 l} \in \mathcal{H}(C, 0)$ and $\beta \in \mathcal{L}(C)$ where $\mathcal{L}(C)$ is the set of square integrable functions on $C$ and

$$
\mathcal{H}(\Omega, a, b)=\left\{z: \Omega \rightarrow \mathcal{R} \mid z \text { and } \nabla z \text { are square integrable over } \Omega, z=a \text { on } \partial \Omega^{d} \text { and } z=b \text { on } \Sigma_{y}\right\}
$$

\subsection{Homogenization under $D_{3}$ symmetry}

Now we consider the $D_{3}$ symmetric unit cell of Figure 5 for the purpose of homogenization, cf. [13]. As explained in subsection 2.2 , we study a body with periodically repeated features, this time in the form of a hexagonal pattern. This means that the properties of the body are invariant to a group of translations for which we may choose, as generators, $\tau_{2}$ and $\tau_{3}$ defined by

$$
\begin{aligned}
& \tau_{2}(\mathbf{x})=\mathbf{x}+\left[\begin{array}{cc}
0 & 1
\end{array}\right]^{T} \\
& \tau_{3}(\mathbf{x})=\mathbf{x}+\left[\begin{array}{ll}
\sqrt{3} / 2 & 1 / 2
\end{array}\right]^{T}
\end{aligned}
$$

Note that invariance with respect to $\tau_{4}$, defined as $\tau_{4}(\mathbf{x})=\mathbf{x}+\left[\begin{array}{ll}-\sqrt{3} / 2 & 1 / 2\end{array}\right]^{T}$, is a mere consequence, as it is for all other elements of the periodicity group.

Before discussing the enforcement of the periodic boundary conditions, however, we examine the unit loads $\chi^{(1)}=\mathbf{e}_{1}=\left[\begin{array}{ll}1 & 0\end{array}\right]^{T}$ and $\chi^{(2)}=\mathbf{e}_{2}=\left[\begin{array}{ll}0 & 1\end{array}\right]^{T}$. Breaking these loads down into their components via Equation 22 and using the analogy from Equation 12 gives

$$
\begin{array}{r}
\chi_{1}^{(1)}=\chi_{2}^{(1)}=\chi_{3}^{(1)^{11}}=\chi_{3}^{(1)^{21}}=\mathbf{0}, \chi_{3}^{(1)^{12}}=-\mathbf{e}_{2} \text { and } \chi_{3}^{(1)^{22}}=\mathbf{e}_{1} \\
\chi_{1}^{(2)}=\chi_{2}^{(2)}=\chi_{3}^{(2)^{12}}=\chi_{3}^{(2)^{22}}=\mathbf{0}, \chi_{3}^{(2)^{11}}=-\chi_{3}^{(1)^{12}}=\mathbf{e}_{2} \text { and } \chi_{3}^{(2)^{21}}=-\chi_{3}^{(1)^{22}}=-\mathbf{e}_{1}
\end{array}
$$

Thus we need not concern ourselves with the $w_{1}^{(i)}$ and $w_{2}^{(i)}$ problems and in fact we only need to solve the family problem for $\mathbf{w}_{3}^{(1)^{2}}=\left[\begin{array}{ll}w_{3}^{(1)^{12}} & w_{3}^{(1)^{22}}\end{array}\right]^{T}$ corresponding to $\chi^{(1)}$ because $\mathbf{w}_{3}^{(2)^{1}}=\left[\begin{array}{ll}w_{3}^{(2)^{11}} & w_{3}^{(2)^{21}}\end{array}\right]^{T}=$ $-\mathbf{w}_{3}^{(1)^{2}}$.

\footnotetext{
${ }^{8}$ Note that solving a coupled problem on the symmetry cell $C$ is equivalent to solving an (uncoupled) problem on a domain twice the size of $C$, e.g. on $C \cup \sigma_{y}(C)$.
} 

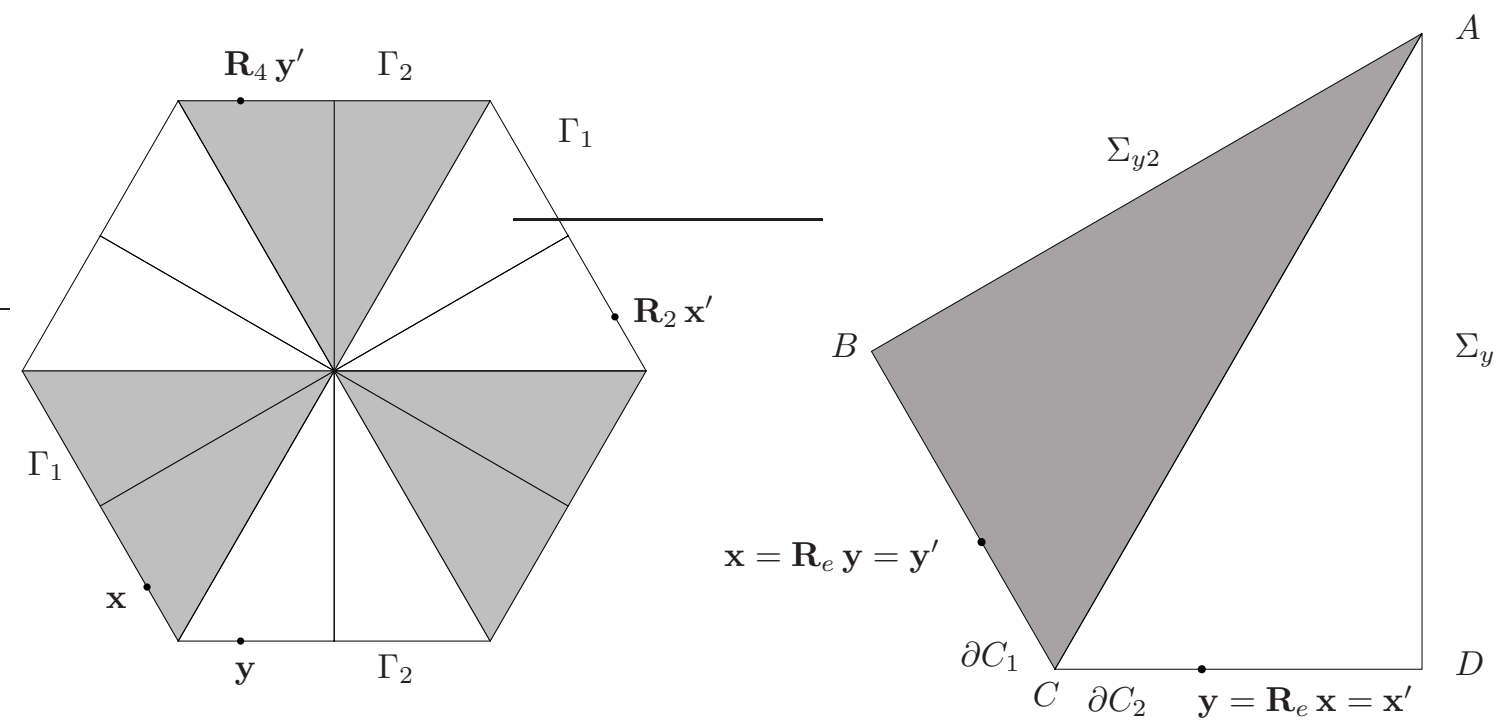

Figure 5: $D_{3}$ unit cell $\Omega$ (left) and its symmetry cell $C$ (right).

Next we consider the boundary conditions corresponding to this periodic setting, following the same ideas as in subsection 2.2. Since we have "cut" a periodicity cell $\Omega$ from the larger body and we allow neither jumps in the response nor in the flux, the resulting boundary conditions on $\partial \Omega$ are twofold. On one hand, $w$ must take equal values on opposite faces ${ }^{9}$ of the cell $\Omega$; on the other, the flux $q$ must take equal values with opposite signs.

Since the cell $\Omega$ is centered at the origin, we can safely replace translations by reflections. For this purpose, we shall use the reflection $\mathbf{R}_{e}$ about the plane with normal vector $\mathbf{e}=[\sqrt{3} / 2,-1 / 2]^{T}$, that is, $\mathbf{R}_{e}=\mathbf{I}-2 \mathbf{e e}^{T}$; it is involutory, i.e. $\mathbf{R}_{e}^{-1}=\mathbf{R}_{e}$. So, $\tau_{2}$ may be replaced by $\mathbf{R}_{4} \mathbf{R}_{e}=\mathbf{R}_{e} \mathbf{R}_{2}$ and $\tau_{3}$ by $\mathbf{R}_{2} \mathbf{R}_{e}=\mathbf{R}_{e} \mathbf{R}_{4}$ (we shall not use $\tau_{4}$ ).

For $\mathbf{x} \in \partial C_{1}=\partial C \cap \Gamma_{1}$ we require that $w\left(\mathbf{R}_{2} \mathbf{R}_{e} \mathbf{x}\right)=w(\mathbf{x})$, cf. Figure 5 . Note the subtle but important difference between the image points used in the $D_{3}$ versus the $D_{2}$ examples. For instance, here the image point $\mathbf{R}_{2} \mathbf{x}^{\prime} \in \Gamma_{2}$ is that of $\mathbf{x}^{\prime}=\mathbf{R}_{e} \mathbf{x} \in \partial C_{2} \subset \Gamma_{2}$ not that of $\mathbf{x} \in \partial C_{1} \subset \Gamma_{1}$. This condition, combined with Equation 23, implies

$$
\begin{aligned}
w_{3}^{j k}(\mathbf{x}) & =w_{3}^{j k}\left(\mathbf{R}_{2} \mathbf{x}^{\prime}\right) \\
& =\sum_{r=1}^{2} \rho_{3}^{j r}\left(\sigma_{2}\right) w_{3}^{r k}\left(\mathbf{x}^{\prime}\right) \\
& =\sum_{r=1}^{2} \rho_{3}^{j r}\left(\sigma_{2}\right) w_{3}^{r k}\left(\mathbf{R}_{e} \mathbf{x}\right)
\end{aligned}
$$

or stated another way, cf. Equation 24

$$
\mathbf{w}_{3}^{k}(\mathbf{x})=\rho_{3}\left(\sigma_{2}\right) \mathbf{w}_{3}^{k}\left(\mathbf{R}_{e} \mathbf{x}\right)
$$

which resembles, but is not identical to Equation 31. ${ }^{10}$ Componentwise,

\footnotetext{
${ }^{9}$ Since the periodicity cell was chosen to be a hexagon, now we have three pairs of "opposite faces" wich relate to each other through either of $\tau_{2}, \tau_{3}$ or $\tau_{4}$. Note that, for this same periodicity group, it is possible to choose a different periodicity cell, e.g. a rhombus, but doing so would break internal symmetries which we want to take advantage of.

10 This type of relation can also appear on cut boundaries, cf. Footnote 2. For example, in the cyclic symmetry $C_{3}$ group $\mathcal{G}=\left\{\sigma_{1}, \sigma_{2}, \sigma_{4}\right\}$ for which the symmetry cell contains the $D_{3}$ symmetry cell $C$ and its mirror image $\mathbf{R}_{y} C$, i.e. the 4 o'clock to 8 o'clock sector, the $\Sigma_{y 2}$ jump condition reads $\mathbf{w}_{i}^{k}(\mathbf{x})=\boldsymbol{\rho}_{i}\left(\sigma_{4}\right) \mathbf{w}_{i}^{k}\left(\mathbf{R}_{y} \mathbf{x}\right)$ which is seen to relate the response on the 4 o'clock and 8 o'clock edges, cf. Figure 5.
} 


$$
\begin{aligned}
& w_{3}^{12}(\mathbf{x})=-\frac{1}{2} w_{3}^{12}\left(\mathbf{R}_{e} \mathbf{x}\right)-\frac{\sqrt{3}}{2} w_{3}^{22}\left(\mathbf{R}_{e} \mathbf{x}\right) \\
& w_{3}^{22}(\mathbf{x})=\frac{\sqrt{3}}{2} w_{3}^{12}\left(\mathbf{R}_{e} \mathbf{x}\right)-\frac{1}{2} w_{3}^{22}\left(\mathbf{R}_{e} \mathbf{x}\right)
\end{aligned}
$$

Similarly, for $\mathbf{y} \in \partial C_{2}$ we require

$$
\mathbf{w}_{3}^{k}(\mathbf{y})=\boldsymbol{\rho}_{3}\left(\sigma_{4}\right) \mathbf{w}_{3}^{k}\left(\mathbf{R}_{e} \mathbf{y}\right)
$$

The fact that for each $\mathbf{x} \in \partial C_{1}$ there exists a $\mathbf{y}=\mathbf{R}_{e} \mathbf{x} \in \partial C_{2}$ leads to a redundancy of the above constraints. Indeed, using this observation in Equations 38 and 40 yields both $\mathbf{w}_{i}^{2}(\mathbf{x})=\boldsymbol{\rho}_{i}\left(\sigma_{2}\right) \mathbf{w}_{i}^{2}\left(\mathbf{R}_{e} \mathbf{x}\right)$ and $\mathbf{w}_{i}^{2}\left(\mathbf{R}_{e} \mathbf{x}\right)=\boldsymbol{\rho}_{i}\left(\sigma_{4}\right) \mathbf{w}_{i}^{2}\left(\mathbf{R}_{e} \mathbf{R}_{e} \mathbf{x}\right)=\boldsymbol{\rho}_{i}\left(\sigma_{4}\right) \mathbf{w}_{i}^{2}(\mathbf{x})$ for $\mathbf{x} \in \partial C_{1}$. Substituting the former in the latter yields the identity $\mathbf{w}_{i}^{2}\left(\mathbf{R}_{e} \mathbf{x}\right)=\boldsymbol{\rho}_{i}\left(\sigma_{4}\right) \boldsymbol{\rho}_{i}\left(\sigma_{2}\right) \mathbf{w}_{i}^{2}\left(\mathbf{R}_{e} \mathbf{x}\right)=\mathbf{w}_{i}^{2}\left(\mathbf{R}_{e} \mathbf{x}\right)$ for $\mathbf{y}=\mathbf{R}_{e} \mathbf{x} \in \partial C_{2}$ where we used the multiplicative property of the irreps and the equality $\mathbf{R}_{4}=\mathbf{R}_{2}^{-1}$. Thusly, satisfying constraint Equation 38 on $\partial C_{1}$ ensures the satisfaction of constraint 40 on $\partial C_{2}$, so we do not need to enforce the latter.

The flux condition writes, for $\mathbf{x} \in \partial C_{1}$,

$$
\mathbf{q}_{3}^{k}(\mathbf{x})=-\mathbf{q}_{3}^{k}\left(\mathbf{R}_{2} \mathbf{x}^{\prime}\right)
$$

We proceed as we did for Equation 38, applying the techniques behind Equation 32 and noting that $\mathbf{n}\left(\mathbf{R}_{2} \mathbf{x}^{\prime}\right)=-\mathbf{n}(\mathbf{x})$, in order to conclude

$$
\mathbf{q}_{3}^{k}(\mathbf{x})=-\boldsymbol{\rho}_{3}\left(\sigma_{2}\right) \mathbf{q}_{3}^{k}\left(\mathbf{R}_{e} \mathbf{x}\right)
$$

The same kind of reasoning leads to the constraint for $\mathbf{y} \in \partial C_{2}$,

$$
\mathbf{q}_{3}^{k}(\mathbf{y})=-\boldsymbol{\rho}_{3}\left(\sigma_{4}\right) \mathbf{q}_{3}^{k}\left(\mathbf{R}_{e} \mathbf{y}\right)
$$

As noted above for $\mathbf{w}_{3}^{k}$, these two conditions are equivalent, so we only need to enforce one of them.

The two unit cell problems on the large domain $\Omega$ are thusly reduced to one coupled unit cell problem on the symmetry cell $C$ which is used to find the response $\mathbf{w}_{3}^{(1)^{2}}=\left[w_{3}^{(1)^{12}} w_{3}^{(1)^{22}}\right]^{T}$ that satisfies

$$
\begin{aligned}
\operatorname{div}\left(\mathbf{k}\left(\nabla w_{3}^{(1)^{12}}+\mathbf{e}_{2}\right)\right. & =0 & & \text { in } C \\
\operatorname{div}\left(\mathbf{k}\left(\nabla w_{3}^{(1)^{22}}-\mathbf{e}_{1}\right)\right. & =0 & & \text { in } C \\
w_{3}^{(1)^{22}} & =0 & & \text { on } \Sigma_{y} \\
q_{3}^{(1)^{12}} & =0 & & \text { on } \Sigma_{y} \\
\sqrt{3} w_{3}^{(1)^{12}}+w_{3}^{(1)^{22}} & =0 & & \text { on } \Sigma_{y 2} \\
q_{3}^{(1)^{12}}-\sqrt{3} q_{3}^{(1)^{22}} & =0 & & \text { on } \Sigma_{y 2} \\
w_{3}^{(1)^{12}}(\mathbf{x}) & =-\frac{1}{2} w_{3}^{(1)^{12}}\left(\mathbf{R}_{e} \mathbf{x}\right)-\frac{\sqrt{3}}{2} w_{3}^{(1)^{22}}\left(\mathbf{R}_{e} \mathbf{x}\right) & & \text { on } \partial C_{1} \\
w_{3}^{(1)^{22}}(\mathbf{x}) & =\frac{\sqrt{3}}{2} w_{3}^{(1)^{12}}\left(\mathbf{R}_{e} \mathbf{x}\right)-\frac{1}{2} w_{3}^{(1)^{22}}\left(\mathbf{R}_{e} \mathbf{x}\right) & & \text { on } \partial C_{1} \\
q_{3}^{(1)^{12}}(\mathbf{x}) & =\frac{1}{2} q_{3}^{(1)^{12}}\left(\mathbf{R}_{e} \mathbf{x}\right)+\frac{\sqrt{3}}{2} q_{3}^{(1)^{22}}\left(\mathbf{R}_{e} \mathbf{x}\right) & & \text { on } \partial C_{1} \\
q_{3}^{(1)^{22}}(\mathbf{x}) & =-\frac{\sqrt{3}}{2} q_{3}^{(1)^{12}}\left(\mathbf{R}_{e} \mathbf{x}\right)+\frac{1}{2} q_{3}^{(1)^{22}}\left(\mathbf{R}_{e} \mathbf{x}\right) & & \text { on } \partial C_{1}
\end{aligned}
$$

We recall that $\mathbf{w}_{3}^{(2)^{1}}=-\mathbf{w}_{3}^{(1)^{2}}$. The finite element approximations of $w_{3}^{(1)^{i 2}}$ are described shortly.

The homogenized material tensor is evaluated with the help of Equation 23, the change of variable theorem, the domain symmetry of $\mathbf{k}$ and the equalities $w^{(1)}(\mathbf{x})=w_{3}^{(1)^{22}}(\mathbf{x})$ and $w^{(2)}(\mathbf{x})=w_{3}^{(1)^{11}}(\mathbf{x})$ which 
follows from Equations 25 and 37, i.e.

$$
\begin{aligned}
& \mathbf{k}_{h}=\frac{1}{\operatorname{vol}(\Omega)} \int_{\Omega} \mathbf{k}(\mathbf{x})\left(\mathbf{I}+\nabla w^{(1)}(\mathbf{x}) \otimes \mathbf{e}_{1}+\nabla w^{(2)}(\mathbf{x}) \otimes \mathbf{e}_{2}\right) d a \\
& =\frac{1}{\operatorname{vol}(\Omega)} \int_{\Omega} \mathbf{k}(\mathbf{x})\left(\mathbf{I}+\nabla w_{3}^{(1)^{22}}(\mathbf{x}) \otimes \mathbf{e}_{1}-\nabla w_{3}^{(1)^{12}}(\mathbf{x}) \otimes \mathbf{e}_{2}\right) d a \\
& =\sum_{g} \frac{1}{\operatorname{vol}(\Omega)} \int_{C} \mathbf{k}\left(\mathbf{R}_{g} \mathbf{x}\right)\left(\mathbf{I}+\nabla w_{3}^{(1)^{22}}\left(\mathbf{R}_{g} \mathbf{x}\right) \otimes \mathbf{e}_{1}-\nabla w_{3}^{(1)^{12}}\left(\mathbf{R}_{g} \mathbf{x}\right) \otimes \mathbf{e}_{2}\right) d a \\
& =\sum_{g} \frac{1}{\operatorname{vol}(\Omega)} \int_{C} \mathbf{R}_{g} \mathbf{k}(\mathbf{x}) \mathbf{R}_{g}^{-1}\left(\mathbf{I}+\mathbf{R}_{g} \sum_{r=1}^{2} \rho_{3}^{2 r}\left(\sigma_{g}\right) \nabla w_{3}^{(1)^{r 2}}(\mathbf{x}) \otimes \mathbf{e}_{1}-\mathbf{R}_{g} \sum_{r=1}^{2} \rho_{3}^{1 r}\left(\sigma_{g}\right) \nabla w_{3}^{(1)^{r 2}}(\mathbf{x}) \otimes \mathbf{e}_{2}\right) d a \\
& =\sum_{g} \frac{1}{\operatorname{vol}(\Omega)} \int_{C} \mathbf{R}_{g} \mathbf{k}(\mathbf{x})\left(\mathbf{R}_{g}^{T}+\sum_{r=1}^{2} \rho_{3}^{2 r}\left(\sigma_{g}\right) \nabla w_{3}^{(1)^{r 2}}(\mathbf{x}) \otimes \mathbf{e}_{1}-\sum_{r=1}^{2} \rho_{3}^{1 r}\left(\sigma_{g}\right) \nabla w_{3}^{(1)^{r 2}}(\mathbf{x}) \otimes \mathbf{e}_{2}\right) d a \\
& =\sum_{g} \frac{1}{\operatorname{vol}(\Omega)} \int_{C} \mathbf{R}_{g} \mathbf{k}(\mathbf{x})\left(\mathbf{R}_{g}^{T}+\left[\begin{array}{ll}
\nabla w_{3}^{(1)^{12}}(\mathbf{x}) & \nabla w_{3}^{(1)^{22}}(\mathbf{x})
\end{array}\right] \boldsymbol{\rho}_{3}\left(\sigma_{g}\right)\left[\begin{array}{ll}
-\mathbf{e}_{2} & \mathbf{e}_{1}
\end{array}\right]^{T}\right) d a
\end{aligned}
$$

For completeness we briefly discuss the finite element solution to the nontrivial $\mathbf{w}_{3}^{(1)^{2}}$. To solve the coupled problem each node $I$ is assigned two-degrees-of-freedom such that $W_{12}^{I} \approx w_{3}^{12}\left(\mathbf{X}^{I}\right)$ and $W_{22}^{I} \approx w_{3}^{22}\left(\mathbf{X}^{I}\right)$ where $\mathbf{X}^{I}$ is the node $I$ location. The formation of the stiffness matrices and load vectors for the $W_{12}^{I}$ and $W_{22}^{I}$ problems is standard.

What is not standard is the enforcement of the constraints. Referring Figure 5 , for nodes $I$ on the $\Sigma_{y}$ surface we enforce the $W_{22}^{I}=0$ constraint which is a standard and warrants no special treatment. For nodes $I$ on the $\Sigma_{y 2}$ surface we enforce the "multi-point" constraint $\sqrt{3} W_{12}^{I}+W_{22}^{I}=0$. And for nodes $I$ on the $\partial C_{1}$ surface we enforce the constraints $W_{12}^{I}-\left(-1 / 2 W_{12}^{J}-\sqrt{3} / 2 W_{22}^{J}\right)=0$ and $W_{22}^{I}-\left(\sqrt{3} / 2 W_{12}^{J}-1 / 2 W_{22}^{J}\right)=0$ where node $J$ has location $\mathbf{X}^{J}=\mathbf{R}_{e} \mathbf{X}^{I}$. E.g. $W_{12}^{B}-\left(-1 / 2 W_{12}^{D}-\sqrt{3} / 2 W_{22}^{D}\right)=0$.

Special care is required at the corner nodes which belong to multiple surfaces. The nodes only have two-degrees-of-freedom so at most two (independent) constraint equations can be enforced. Referring to Figure 5 we see that node $A$ belongs to both $\Sigma_{y}$ and $\Sigma_{y 2}$ and thusly it must satisfy two linearly independent constraint equations $W_{12}^{A}=0$ and $\sqrt{3} W_{12}^{A}+W_{22}^{A}=0$ which requires $W_{12}^{A}=W_{22}^{A}=0$ which is a standard constraint. Node $C$ belongs to $\partial C_{1}$ and $\partial C_{2}$ whence $\mathbf{R}_{e} \mathbf{x}=\mathbf{x}=\mathbf{X}^{C}$ whereby constraint Equation 40 requires $\mathbf{w}_{3}^{2}\left(\mathbf{X}^{C}\right)=\boldsymbol{\rho}_{3}\left(\sigma_{2}\right) \mathbf{w}_{3}^{2}\left(\mathbf{X}^{C}\right)$ or $W_{12}^{C}=W_{22}^{C}=0$ (since $\boldsymbol{\rho}_{3}\left(\sigma_{2}\right)$ is nonsingular), which is also a standard constraint.

Node $B$ belongs $\Sigma_{y 2}$ and $\partial C_{1}$ while node $D$ belongs $\Sigma_{y}$ and $\partial C_{2}$ and thus nodes $B$ and $D$ are linked by $1\left(\right.$ on $\left.\Sigma_{y 2}\right)+2\left(\right.$ on $\left.\partial C_{1}\right)+1\left(\right.$ on $\left.\Sigma_{y}\right)=4$ constraint equations. However, only three of the four equations are independent. Indeed, twice the $\Sigma_{y}$ equation $W_{22}^{D}=0$ plus the $\Sigma_{y 2}$ equation $\sqrt{3} W_{12}^{B}+W_{22}^{B}=0$ less $\sqrt{3}$ times the $\partial C_{1}$ Equation $39 W_{12}^{B}-\left(-1 / 2 W_{12}^{D}-\sqrt{3} / 2 W_{22}^{D}\right)=0$ yields the $\partial C_{1}$ Equation $39 W_{22}^{B}-\left(\sqrt{3} / 2 W_{12}^{D}-\right.$ $\left.1 / 2 W_{22}^{D}\right)=0$. Consequently we enforce the $\Sigma_{y}$ and $\Sigma_{2 y}$ equations and just one of the $\partial C_{1}$ equations.

The coupled equations, e.g. Equation 35, result in a linear matrix equation

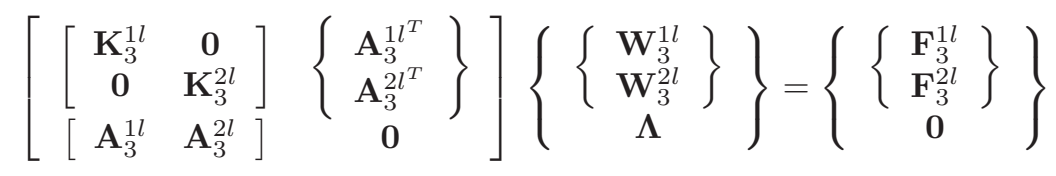

Referring to Equation 35, the matrices $\mathbf{K}_{3}^{1 l}$ and $\mathbf{K}_{3}^{2 l}$ are formed by the first two integrals on the left hand side, $\mathbf{A}_{3}^{1 l}$ and $\mathbf{A}_{3}^{2 l}$ are formed by the third integral on the left hand side, $\mathbf{A}_{3}^{1 l^{T}}$ and $\mathbf{A}_{3}^{2 l^{T}}$ are formed by the last integral on the left hand side, i.e. the multi-point constraints, and $\mathbf{F}_{3}^{1 l}$ and $\mathbf{F}_{3}^{2 l}$ are formed by the terms in brackets on the right hand side. $\mathbf{W}_{3}^{1 l}$ and $\mathbf{W}_{3}^{2 l}$ are the usual nodal response vectors and $\boldsymbol{\Lambda}$ is the vector of nodal Lagrange multipliers that is required to enforce the multi-point constraints.

As seen above, we need to solve a linear system of the form

$$
\left[\begin{array}{cc}
\mathbf{K} & \mathbf{A}^{T} \\
\mathbf{A} & \mathbf{0}
\end{array}\right]\left\{\begin{array}{c}
\mathbf{W} \\
\boldsymbol{\Lambda}
\end{array}\right\}=\left\{\begin{array}{l}
\mathbf{F} \\
\mathbf{0}
\end{array}\right\}
$$

where $\mathbf{K}$ is block diagonal symmetric positive definite. One way to attack this problem is to 
1. Rearrange the top row and replace the bottom row by multiplying the top row by $\mathbf{A} \mathbf{K}^{-1}$ and subtracting the bottom row from that result to obtain the equivalent system

$$
\begin{aligned}
\mathbf{W} & =\overbrace{\mathbf{K}^{-1} \mathbf{F}}^{=\mathbf{z}}-\overbrace{\mathbf{K}^{-1} \mathbf{A}^{T}}^{=\mathbf{Z}} \boldsymbol{\Lambda} \\
\mathbf{A} \underbrace{\mathbf{K}^{-1} \mathbf{A}^{T}}_{=\mathbf{Z}} \boldsymbol{\Lambda} & =\mathbf{A} \underbrace{\mathbf{K}^{-1} \mathbf{F}}_{=\mathbf{z}}
\end{aligned}
$$

2. Find the $\mathbf{Z}=\mathbf{K}^{-1} \mathbf{A}^{T}$ that satisfies $\mathbf{K} \mathbf{Z}=\mathbf{A}^{T}$ by factoring $\mathbf{K}$ and performing repeated backsolves to compute each column of $\mathbf{Z}$. Benefits from the block diagonal structure of $\mathbf{K}$ should be utilized to hasten these computations.

3. Find the $\mathbf{z}=\mathbf{K}^{-1} \mathbf{F}$ that satisfies $\mathbf{K} \mathbf{z}=\mathbf{F}$ by performing an additional backsolve on $\mathbf{K}$.

4. Find $\boldsymbol{\Lambda}$ by solving Equation 41.2 which now is expressed as $\mathbf{A} \mathbf{Z} \mathbf{\Lambda}=\mathbf{A} \mathbf{z}$.

5. Compute $\mathbf{W}$ of Equation 41.1 which now is expressed as $\mathbf{W}=\mathbf{z}-\mathbf{Z} \mathbf{\Lambda}$.

Further computational gains are obtained if we only enforce the $\Sigma_{y} \cap \Sigma_{y 2}$ and $\partial C_{1} \cap \partial C_{2}$ constraints, i.e. $W_{12}^{A}=W_{22}^{A}=W_{12}^{C}=W_{22}^{C}=0$ in the "standard way" and enforce the $w_{3}^{(1)^{22}}=0$ constraint for the nodes on the interior of edge $\Sigma_{y}$ via Lagrange multipliers. In this way, $\mathbf{K}_{3}^{1 l}=\mathbf{K}_{3}^{2 l}$ and hence we only need to assemble and factor one stiffness matrix since $\mathbf{K}^{-1}=\operatorname{diag}\left(\mathbf{K}_{3}^{1 l^{-1}}, \mathbf{K}_{3}^{2 l^{-1}}\right)$.

\section{$3 \quad$ Vector valued elliptic problems}

Now we are concerned with solving an elastostatics elliptic boundary value problem of finding the response $\mathbf{w} \in \mathcal{H}\left(\Omega, \mathbf{w}^{p}\right)$ such that

$$
\int_{\Omega} \nabla \mathbf{v} \cdot \mathbb{C}[\nabla \mathbf{w}] d v=\int_{\Omega}(\nabla \mathbf{v} \cdot \mathbb{C}[\boldsymbol{\chi}]+\mathbf{v} \cdot \mathbf{f}) d v+\int_{\partial \Omega^{n}} \mathbf{v} \cdot \mathbf{t}^{p} d a
$$

for all $\mathbf{v} \in \mathcal{H}(\Omega, \mathbf{0})$ where

$$
\mathcal{H}\left(\Omega, \mathbf{z}^{p}\right)=\left\{\mathbf{z}: \Omega \rightarrow \mathcal{R}^{2} \mid \mathbf{z} \text { and } \nabla \mathbf{z} \text { are square integrable over } \Omega \text { and } \mathbf{z}=\mathbf{z}^{p} \text { on } \partial \Omega^{d}\right\}
$$

The body $\Omega$ is comprised of a material that is described by the elasticity tensor $\mathbb{C}$ and it is subjected to the body load $\mathbf{f}$ and initial strain $\chi$. The boundary of $\Omega$ is again partitioned into the complementary surfaces $\partial \Omega^{d}$ and $\partial \Omega^{n}$ over which the displacement $\mathbf{w}^{p}$ and traction $\mathbf{t}^{p}=\mathbb{C}[\nabla w-\chi] \mathbf{n}$ are prescribed.

We are again interested in bodies that have domain symmetry with respect to the group of orthogonal transformations $\mathcal{G}=\left\{\mathbf{R}_{1}, \mathbf{R}_{2}, \cdots\right\}$, cf. Figure 2. This also means that the elasticity tensor $\mathbb{C}$ at $\mathbf{x}$ and $\mathbf{R}_{i} \mathbf{x}$ satisfy $\mathbb{C}(\mathbf{x})=\left(\mathbf{R}_{i} \otimes \mathbf{R}_{i}\right)^{-1} \mathbb{C}\left(\mathbf{R}_{i} \mathbf{x}\right)\left(\mathbf{R}_{i} \otimes \mathbf{R}_{i}\right)$. Here $\nabla$ is the conjugation product defined such that $\mathbf{A} \mathbf{B} \mathbf{C}^{T}=(\mathbf{A} \otimes \mathbf{C}) \mathbf{B}$ for all 2-tensors $\mathbf{A}, \mathbf{B}$ and $\mathbf{C}$. Thusly $\mathbf{A} \otimes \mathbf{C}$ is a 4-tensor; it satisfies $(\mathbf{A} \otimes \mathbf{B})^{T}=$ $\mathbf{A}^{T} \otimes \mathbf{B}^{T},(\mathbf{A} \otimes \mathbf{B})(\mathbf{C} \otimes \mathbf{D})=(\mathbf{A} \mathbf{C}) \otimes(\mathbf{B} \mathbf{D})$ where $\mathbf{D}$ is also a 2 -tensor, and $(\mathbf{A} \otimes \mathbf{B})^{-1}=\mathbf{A}^{-1} \otimes \mathbf{B}^{-1}$ provided that $\mathbf{A}$ and $\mathbf{B}$ are invertible.

To ease the presentation we also utilize the matrix Kronecker product defined as the $m r \times n s$ matrix

$$
\mathbf{A} \odot \mathbf{B}=\left[\begin{array}{cccc}
A_{11} \mathbf{B} & A_{12} \mathbf{B} & \ldots & A_{1 n} \mathbf{B} \\
A_{21} \mathbf{B} & A_{22} \mathbf{B} & \ldots & A_{2 n} \mathbf{B} \\
\vdots & \vdots & \ddots & \vdots \\
A_{m 1} \mathbf{B} & A_{m 2} \mathbf{B} & \ldots & A_{m n} \mathbf{B}
\end{array}\right]
$$

for any $m \times n$ matrix $\mathbf{A}$ and $r \times s$ matrix $\mathbf{B}$. It can be verified that $(\mathbf{A} \odot \mathbf{B})(\mathbf{C} \odot \mathbf{D})=\mathbf{A ~} \mathbf{C} \odot \mathbf{B} \mathbf{D}$ assuming the matrices $\mathbf{A}, \mathbf{B}, \mathbf{C}$ and $\mathbf{D}$ are suitably compatible and that $(\mathbf{A} \odot \mathbf{B})^{T}=\left(\mathbf{A}^{T} \odot \mathbf{B}^{T}\right)$. And if $\mathbf{A}$ and $\mathbf{B}$ are square matrices of dimension $m$ and $n$ with eigenvalues $\alpha_{i}$ for $i=1,2, \ldots, m$ and $\beta_{j}$ for $j=1,2, \ldots, n$, then the eigenvalues of $\mathbf{A} \odot \mathbf{B}$ are $\alpha_{i} \beta_{j}$ for $i=1,2, \ldots, m$ and $j=1,2, \ldots, n$. 


\subsection{Abelian symmetry}

For abelian groups we construct the projectors such that for any vector-valued function on the symmetry domain $\mathbf{w}: \Omega \rightarrow \mathcal{R}^{2}$ we have

$$
P_{i} \mathbf{w}(\mathbf{x})=\frac{1}{n} \sum_{g \in \mathcal{G}} \rho_{i}\left(\sigma_{g}\right) \mathbf{R}_{g} \mathbf{w}\left(\mathbf{R}_{g}^{-1} \mathbf{x}\right)
$$

where the sum is over the group elements. From the above we also see that

$$
\begin{aligned}
P_{i} \mathbf{w}\left(\mathbf{R}_{h}^{-1} \mathbf{x}\right) & =\frac{1}{n} \sum_{g \in \mathcal{G}} \rho_{i}\left(\sigma_{g}\right) \mathbf{R}_{g} \mathbf{w}\left(\mathbf{R}_{g}^{-1}\left(\mathbf{R}_{h}^{-1} \mathbf{x}\right)\right) \\
& =\frac{1}{n} \sum_{g \in \mathcal{G}} \overbrace{\rho_{i}\left(\sigma_{h}^{-1} \sigma_{h}\right)}^{=1} \rho_{i}\left(\sigma_{g}\right) \overbrace{\mathbf{R}_{h}^{-1} \mathbf{R}_{h}}^{\mathbf{I}} \mathbf{R}_{g} \mathbf{w}\left(\left(\mathbf{R}_{h} \mathbf{R}_{g}\right)^{-1} \mathbf{x}\right) \\
& =\left[\rho_{i}\left(\sigma_{h}\right)\right]^{T} \mathbf{R}_{h}^{-1} \frac{1}{n} \sum_{g \in \mathcal{G}} \rho_{i}\left(\sigma_{h} \sigma_{g}\right)\left(\mathbf{R}_{h} \mathbf{R}_{g}\right) \mathbf{w}\left(\left(\mathbf{R}_{h} \mathbf{R}_{g}\right)^{-1} \mathbf{x}\right) \\
& =\rho_{i}\left(\sigma_{h}\right) \mathbf{R}_{h}^{-1} P_{i} \mathbf{w}(\mathbf{x})
\end{aligned}
$$

Using the orthogonality of the irreps and manipulating the above also gives

$$
\begin{aligned}
P_{i} \mathbf{w}\left(\mathbf{R}_{h} \mathbf{x}\right) & =\rho_{i}\left(\sigma_{h}^{-1}\right) \mathbf{R}_{h} P_{i} \mathbf{w}(\mathbf{x}) \\
& =\rho_{i}\left(\sigma_{h}\right) \mathbf{R}_{h} P_{i} \mathbf{w}(\mathbf{x})
\end{aligned}
$$

Upon combining Equations 43 and 44 we find

$$
\begin{aligned}
P_{j} P_{i} \mathbf{w}(\mathbf{x}) & =\frac{1}{n} \sum_{g \in \mathcal{G}} \rho_{j}\left(\sigma_{g}\right) \mathbf{R}_{g} P_{i} \mathbf{w}\left(\mathbf{R}_{g}^{-1} \mathbf{x}\right) \\
& =\frac{1}{n} \sum_{g \in \mathcal{G}} \rho_{j}\left(\sigma_{g}\right) \rho_{i}\left(\sigma_{g}\right) \mathbf{R}_{g} \mathbf{R}_{g}^{-1} P_{i} \mathbf{w}(\mathbf{x}) \\
& =\delta_{i j} P_{i} \mathbf{w}(\mathbf{x})
\end{aligned}
$$

i.e. we again have orthogonal projectors. And as expected, we have

$$
\mathbf{w}(\mathbf{x})=\sum_{i=1}^{n} P_{i} \mathbf{w}(\mathbf{x}),
$$

i.e. we again have the orthogonal subspaces $P_{i} \mathcal{H}(\Omega)$ such that $\mathcal{H}(\Omega)=\bigoplus_{i=1}^{n} P_{i} \mathcal{H}(\Omega)$. The proof is analogous to that of Equation 10.

Differentiating Equations 43 and 44 gives

$$
\begin{aligned}
\nabla P_{i} \mathbf{w}(\mathbf{x}) & =\frac{1}{n} \sum_{g \in \mathcal{G}} \rho_{i}\left(\sigma_{g}\right) \mathbf{R}_{g} \nabla \mathbf{w}\left(\mathbf{R}_{g}^{-1} \mathbf{x}\right) \mathbf{R}_{g}^{-1} \\
\nabla P_{i} \mathbf{w}\left(\mathbf{R}_{g}^{-1} \mathbf{x}\right) & =\rho_{i}\left(\sigma_{g}\right) \mathbf{R}_{g}^{-1} \nabla P_{i} \mathbf{w}(\mathbf{x}) \mathbf{R}_{g}
\end{aligned}
$$

Noting the tensor like analogy between $\nabla \mathbf{w}$ and $\chi$ we define and subsequently find

$$
\begin{aligned}
P_{i} \chi(\mathbf{x}) & =\frac{1}{n} \sum_{g \in \mathcal{G}} \rho_{i}\left(\sigma_{g}\right) \mathbf{R}_{g} \chi\left(\mathbf{R}_{g}^{-1} \mathbf{x}\right) \mathbf{R}_{g}^{-1} \\
P_{i} \chi\left(\mathbf{R}_{g}^{-1} \mathbf{x}\right) & =\rho_{i}\left(\sigma_{g}\right) \mathbf{R}_{g}^{-1} P_{i} \chi(\mathbf{x}) \mathbf{R}_{g}
\end{aligned}
$$

Arguing as we do to obtain Equation 45, it is seen that the above Equations 48 and 49 can also be stated as

$$
\begin{aligned}
\nabla P_{i} \mathbf{w}\left(\mathbf{R}_{g} \mathbf{x}\right) & =\rho_{i}\left(\sigma_{g}\right) \mathbf{R}_{g} \nabla P_{i} \mathbf{w}(\mathbf{x}) \mathbf{R}_{g}^{-1} \\
P_{i} \chi\left(\mathbf{R}_{g} \mathbf{x}\right) & =\rho_{i}\left(\sigma_{g}\right) \mathbf{R}_{g} P_{i} \chi(\mathbf{x}) \mathbf{R}_{g}^{-1}
\end{aligned}
$$


We return to the integral Equation 42. Since it must hold for arbitrary $\mathbf{v} \in \mathcal{H}\left(\Omega, \mathbf{w}^{p}\right)$ it must hold for some $\mathbf{v}_{i}=P_{i} \mathbf{v}$. Substituting this choice into Equation 42 gives

$$
\begin{aligned}
& \int_{\Omega} \nabla \mathbf{v}_{i}(\mathbf{x}) \cdot \mathbb{C}(\mathbf{x})[\nabla \mathbf{w}(\mathbf{x})] d v=\int_{\Omega}\left(\nabla \mathbf{v}_{i}(\mathbf{x}) \cdot \mathbb{C}(\mathbf{x})[\chi(\mathbf{x})]+\mathbf{v}_{i}(\mathbf{x}) \cdot \mathbf{f}(\mathbf{x})\right) d v+ \\
& \int_{\partial \Omega^{n}} \mathbf{v}_{i}(\mathbf{x}) \cdot \mathbf{t}^{p}(\mathbf{x}) d a \\
& \sum_{g \in \mathcal{G}} \int_{\mathbf{R}_{g}^{-1}(C)} \nabla \mathbf{v}_{i}(\mathbf{x}) \cdot \mathbb{C}(\mathbf{x})[\nabla \mathbf{w}(\mathbf{x})] d v=\sum_{g \in \mathcal{G}} \int_{\mathbf{R}_{g}^{-1}(C)}\left(\nabla \mathbf{v}_{i}(\mathbf{x}) \cdot \mathbb{C}(\mathbf{x})[\chi(\mathbf{x})]+\mathbf{v}_{i}(\mathbf{x}) \cdot \mathbf{f}(\mathbf{x})\right) d v+ \\
& \sum_{g \in \mathcal{G}} \int_{\mathbf{R}_{g}^{-1}\left(\partial C^{n}\right)} \mathbf{v}_{i}(\mathbf{x}) \cdot \mathbf{t}^{p}(\mathbf{x}) d a \\
& \sum_{g \in \mathcal{G}} \int_{C} \nabla \mathbf{v}_{i}\left(\mathbf{R}_{g}^{-1} \mathbf{x}\right) \cdot \mathbb{C}\left(\mathbf{R}_{g}^{-1} \mathbf{x}\right)\left[\nabla \mathbf{w}\left(\mathbf{R}_{g}^{-1} \mathbf{x}\right)\right] d v=\sum_{g \in \mathcal{G}} \int_{C}\left(\nabla \mathbf{v}_{i}\left(\mathbf{R}_{g}^{-1} \mathbf{x}\right) \cdot \mathbb{C}\left(\mathbf{R}_{g}^{-1} \mathbf{x}\right)\left[\chi\left(\mathbf{R}_{g}^{-1} \mathbf{x}\right)\right]+\right. \\
& \left.\mathbf{v}_{i}\left(\mathbf{R}_{g}^{-1} \mathbf{x}\right) \cdot \mathbf{f}\left(\mathbf{R}_{g}^{-1} \mathbf{x}\right)\right) d v+ \\
& \sum_{g \in \mathcal{G}} \int_{\partial C^{n}} \mathbf{v}_{i}\left(\mathbf{R}_{g}^{-1} \mathbf{x}\right) \cdot \mathbf{t}^{p}\left(\mathbf{R}_{g}^{-1} \mathbf{x}\right) d a \\
& \sum_{g \in \mathcal{G}} \int_{C} \rho_{i}\left(\sigma_{g}\right) \mathbf{R}_{g}^{-1} \nabla \mathbf{v}_{i}(\mathbf{x}) \mathbf{R}_{g} . \\
& \left(\mathbf{R}_{g} \otimes \mathbf{R}_{g}\right)^{-1} \mathbb{C}(\mathbf{x})\left(\mathbf{R}_{g} \otimes \mathbf{R}_{g}\right)\left[\nabla \mathbf{w}\left(\mathbf{R}_{g}^{-1} \mathbf{x}\right)\right] d v \quad=\sum_{g \in \mathcal{G}} \int_{C}\left(\rho_{i}\left(\sigma_{g}\right) \mathbf{R}_{g}^{-1} \nabla \mathbf{v}_{i}(\mathbf{x}) \mathbf{R}_{g} .\right. \\
& \left(\mathbf{R}_{g} \otimes \mathbf{R}_{g}\right)^{-1} \mathbb{C}(\mathbf{x})\left(\mathbf{R}_{g} \otimes \mathbf{R}_{g}\right)\left[\chi\left(\mathbf{R}_{g}^{-1} \mathbf{x}\right)\right]+ \\
& \left.\rho_{i}\left(\sigma_{g}\right) \mathbf{R}_{g}^{-1} \mathbf{v}_{i}(\mathbf{x}) \cdot \mathbf{f}\left(\mathbf{R}_{g}^{-1} \mathbf{x}\right)\right) d v+ \\
& \sum_{g \in \mathcal{G}} \int_{\partial C^{n}} \rho_{i}\left(\sigma_{g}\right) \mathbf{R}_{g}^{-1} \mathbf{v}_{i}(\mathbf{x}) \cdot \mathbf{t}^{p}\left(\mathbf{R}_{g}^{-1} \mathbf{x}\right) d a \\
& \sum_{g \in \mathcal{G}} \int_{C} \nabla \mathbf{v}_{i}(\mathbf{x}) \cdot \mathbb{C}(\mathbf{x})\left[\rho_{i}\left(\sigma_{g}\right) \mathbf{R}_{g} \nabla \mathbf{w}\left(\mathbf{R}_{g}^{-1} \mathbf{x}\right) \mathbf{R}_{g}^{-1}\right] d v=\sum_{g \in \mathcal{G}} \int_{C}\left(\nabla \mathbf{v}_{i}(\mathbf{x}) \cdot \mathbb{C}(\mathbf{x})\left[\rho_{i}\left(\sigma_{g}\right) \mathbf{R}_{g} \chi\left(\mathbf{R}_{g}^{-1} \mathbf{x}\right) \mathbf{R}_{g}^{-1}\right]+\right. \\
& \left.\mathbf{v}_{i}(\mathbf{x}) \cdot \rho_{i}\left(\sigma_{g}\right) \mathbf{R}_{g} \mathbf{f}\left(\mathbf{R}_{g}^{-1} \mathbf{x}\right)\right) d v+ \\
& \sum_{g \in \mathcal{G}} \int_{\partial C^{n}} \mathbf{v}_{i}(\mathbf{x}) \cdot \rho_{i}\left(\sigma_{g}\right) \mathbf{R}_{g} \mathbf{t}^{p}\left(\mathbf{R}_{g}^{-1} \mathbf{x}\right) d a \\
& n \int_{C} \nabla \mathbf{v}_{i}(\mathbf{x}) \cdot \mathbb{C}(\mathbf{x})\left[\nabla \mathbf{w}_{i}(\mathbf{x})\right] d v=n \int_{C}\left(\nabla \mathbf{v}_{i}(\mathbf{x}) \cdot \mathbb{C}(\mathbf{x})\left[\chi_{i}(\mathbf{x})\right]+\mathbf{v}_{i}(\mathbf{x}) \cdot \mathbf{f}_{i}(\mathbf{x})\right) d v+ \\
& n \int_{\partial C^{n}} \mathbf{v}_{i}(\mathbf{x}) \cdot \mathbf{t}_{i}^{p}(\mathbf{x}) d a
\end{aligned}
$$

where $\mathbf{w}_{i}=P_{i} \mathbf{w}$ etc. To obtain the above we used the summation of integration, change-of-variable theorem, Equations $48-49$ and the domain symmetry of $\mathbb{C}$.

As seen before in the scalar case, the projection property is such that our choice of $\mathbf{v}_{i}$ renders an integral expression which only contains the $\mathbf{w}_{i}$ component of $\mathbf{w}$. So using Equation 47 to express $\mathbf{v}=\sum_{i=1}^{n} \mathbf{v}_{i}$ and substituting into Equation 42 we are again able to evaluate the response $\mathbf{w}=\sum_{i=1}^{n} \mathbf{w}_{i}$ one component at a time. Summarizing, the boundary value problem of Equation 42 is now converted into $n$ problems which read: find the response $\mathbf{w}_{i} \in \mathcal{H}\left(C, \mathbf{w}_{i}^{p}\right)$ such that

$$
\int_{C} \nabla \mathbf{v}_{i} \cdot \mathbb{C}\left[\nabla \mathbf{w}_{i}\right] d v=\int_{C}\left(\nabla \mathbf{v}_{i} \cdot \mathbb{C}\left[\boldsymbol{\chi}_{i}\right]+\mathbf{v}_{i} \cdot \mathbf{f}_{i}\right) d v+\int_{\partial C^{n}} \mathbf{v}_{i} \cdot \mathbf{t}_{i}^{p} d a
$$

for all $\mathbf{v}_{i} \in \mathcal{H}(C, \mathbf{0})$.

We now consider the cut surfaces of $C$ and enforce the jump conditions

$$
\begin{aligned}
\llbracket \mathbf{w}_{i}(\mathbf{x}) \rrbracket & =\mathbf{0} \\
\llbracket \mathbb{C}(\mathbf{x})\left[\nabla \mathbf{w}_{i}(\mathbf{x})-\chi_{i}(\mathbf{x})\right] \rrbracket \mathbf{n}(\mathbf{x}) & =\mathbf{0}
\end{aligned}
$$


Considering the $D_{2}$ case and substituting Equations 45 and 50 into the above jump conditions over $\Sigma_{x}$ gives

$$
\begin{aligned}
\mathbf{w}_{i}(\mathbf{x}) & =\mathbf{w}_{i}\left(\mathbf{R}_{x} \mathbf{x}\right) \\
& =\rho_{i}\left(\sigma_{x}\right) \mathbf{R}_{x} \mathbf{w}_{i}(\mathbf{x}) \\
& =\rho_{i}\left(\sigma_{x}\right)\left\{\begin{array}{c}
w_{i_{x}}(\mathbf{x}) \\
-w_{i_{y}}(\mathbf{x})
\end{array}\right\}
\end{aligned}
$$

and

$$
\begin{aligned}
\mathbb{C}(\mathbf{x})\left[\nabla \mathbf{w}_{i}(\mathbf{x})-\chi_{i}(\mathbf{x})\right] \mathbf{n}(\mathbf{x}) & =\mathbb{C}\left(\mathbf{R}_{x} \mathbf{x}\right)\left[\nabla \mathbf{w}_{i}\left(\mathbf{R}_{x} \mathbf{x}\right)-\chi_{i}\left(\mathbf{R}_{x} \mathbf{x}\right)\right] \mathbf{n}(\mathbf{x}) \\
& =\left(\mathbf{R}_{x} \boldsymbol{\nabla} \mathbf{R}_{x}\right) \mathbb{C}(\mathbf{x})\left(\mathbf{R}_{x} \boldsymbol{\nabla} \mathbf{R}_{x}\right)^{-1}\left[\rho_{i}\left(\sigma_{x}\right) \mathbf{R}_{x}\left(\nabla \mathbf{w}_{i}(\mathbf{x})-\chi_{i}(\mathbf{x})\right) \mathbf{R}_{x}^{-1}\right] \mathbf{n}(\mathbf{x}) \\
& \left.=\rho_{i}\left(\sigma_{x}\right) \mathbf{R}_{x} \mathbb{C}(\mathbf{x})\left[\nabla \mathbf{w}_{i}(\mathbf{x})-\chi_{i}(\mathbf{x})\right)\right] \mathbf{R}_{x}^{T} \mathbf{n}(\mathbf{x}) \\
& =-\rho_{i}\left(\sigma_{x}\right) \mathbf{R}_{x} \mathbb{C}(\mathbf{x})\left[\nabla \mathbf{w}_{i}(\mathbf{x})-\chi_{i}(\mathbf{x})\right] \mathbf{n}(\mathbf{x}) \\
& =-\rho_{i}\left(\sigma_{x}\right) \mathbf{R}_{x} \mathbf{t}(\mathbf{x}) \\
& =\rho_{i}\left(\sigma_{x}\right)\left\{\begin{array}{c}
-t_{i_{x}} \\
t_{i_{y}}
\end{array}\right\}
\end{aligned}
$$

where we use the fact that $\mathbf{R}_{x}^{T} \mathbf{n}(\mathbf{x})=-\mathbf{n}(\mathbf{x})$ for $\mathbf{x} \in \Sigma_{x}$ and define the normal traction $\mathbf{t}_{i}=\left[\begin{array}{ll}t_{i_{x}} & t_{i_{y}}\end{array}\right]^{T}=$ $\mathbb{C}\left[\nabla \mathbf{w}_{i}-\boldsymbol{\chi}_{i}\right] \mathbf{n}$. Mimicking the development of Equation 18, we express Equations 53 and 54 as

$$
\begin{aligned}
\frac{1}{2}\left(\mathbf{I}-\rho_{i}\left(\sigma_{x}\right) \mathbf{R}_{x}\right) \mathbf{w}_{i}(\mathbf{x}) & =\mathbf{0} \\
\frac{1}{2}\left(\mathbf{I}+\rho_{i}\left(\sigma_{x}\right) \mathbf{R}_{x}\right) \mathbf{t}_{i}(\mathbf{x}) & =\mathbf{0}
\end{aligned}
$$

And, as per the discussion following Equation 18, we again encounter the complementary projections $\frac{1}{2}(\mathbf{I}-$ $\left.\rho_{i}\left(\sigma_{x}\right) \mathbf{R}_{x}\right)$ and $\frac{1}{2}\left(\mathbf{I}+\rho_{i}\left(\sigma_{x}\right) \mathbf{R}_{x}\right)$.

For $i=1,2$ we have $\rho_{i}\left(\sigma_{x}\right)=1$ so the first equation above requires $w_{i_{y}}(\mathbf{x})=0$ while the second requires $t_{i_{x}}(\mathbf{x})=0$. For $i=3,4$ we have $\rho_{i}\left(\sigma_{y}\right)=-1$ so that the first equation above requires $w_{i_{x}}(\mathbf{x})=0$ while the second necessitates that $t_{i_{y}}(\mathbf{x})=0$. Performing similar derivation for $\mathbf{x} \in \Sigma_{y}$ we find $w_{i_{x}}(\mathbf{x})=0$ and $t_{i_{y}}(\mathbf{x})=0$ for $i=1,3$ and $w_{i_{y}}(\mathbf{x})=0$ and $t_{i_{x}}(\mathbf{x})=0$ for $i=2,4$.

Summarizing, for the $D_{2}$ symmetric domain we solve $n=4$ boundary value problems on $C$ for the $\mathbf{w}_{i}$ of 
the form

$$
\begin{aligned}
& \operatorname{div}\left(\mathbb{C}\left[\nabla \mathbf{w}_{i}-\boldsymbol{\chi}_{i}\right]+\mathbf{f}_{i}=0 \quad \text { in } C \text { and } i=1,2,3,4\right. \\
& \mathbf{w}_{i}=\mathbf{w}_{i}^{p} \quad \text { on } \partial C^{d} \text { and } i=1,2,3,4 \\
& \mathbf{t}_{i}=\mathbf{t}_{i}^{p} \quad \text { on } \partial C^{n} \text { and } i=1,2,3,4
\end{aligned}
$$$$
\begin{aligned}
& \left\{\begin{array}{l}
\left\{\begin{array}{l}
w_{1_{y}} \\
t_{1_{x}}
\end{array}\right\} \\
\left\{\begin{array}{l}
w_{2_{y}} \\
t_{2_{x}}
\end{array}\right\} \\
\left\{\begin{array}{l}
w_{3_{x}} \\
t_{3_{y}}
\end{array}\right\} \\
\left\{\begin{array}{c}
w_{4_{x}} \\
t_{4_{y}}
\end{array}\right\}
\end{array}\right\}=\mathbf{0} \quad \text { on } \Sigma_{x} \\
& \left\{\begin{array}{l}
\left\{\begin{array}{l}
w_{1_{x}} \\
t_{1_{y}}
\end{array}\right\} \\
\left\{\begin{array}{l}
w_{2_{y}} \\
t_{2_{x}}
\end{array}\right\} \\
\left\{\begin{array}{l}
w_{3_{x}} \\
t_{3_{y}}
\end{array}\right\} \\
\left\{\begin{array}{l}
w_{4_{y}} \\
t_{4_{x}}
\end{array}\right\}
\end{array}\right\}=\mathbf{0} \quad \text { on } \Sigma_{y}
\end{aligned}
$$

As before, the applied traction conditions, e.g. $t_{4_{x}}=0$ on $\Sigma_{y}$, are automatically accommodated in the weak formulation, cf. Equation 52.

These responses $w_{i}$ obtained by solving the above equations are used to obtain $w_{i}$ throughout $\Omega$ via Equation 45 and summed via Equation 47 to evaluate w, i.e.

$$
\mathbf{w}\left(\mathbf{R}_{g} \mathbf{x}\right)=\sum_{i=1}^{n} \mathbf{w}_{i}\left(\mathbf{R}_{g} \mathbf{x}\right)=\sum_{i=1}^{n} \rho_{i}\left(\sigma_{g}\right) \mathbf{R}_{g} \mathbf{w}_{i}(\mathbf{x})
$$

\subsection{Homogenization under $D_{2}$ symmetry}

We now consider the $D_{2}$ symmetric unit cell of Figure 3 for the purpose of homogenization. The "unit loads" for homogenization are $\boldsymbol{\chi}^{(11)}=\mathbf{E}_{11}=\mathbf{e}_{1} \otimes \mathbf{e}_{1}=\left[\begin{array}{ll}1 & 0 \\ 0 & 0\end{array}\right], \boldsymbol{\chi}^{(21)}=\mathbf{E}_{21}=\mathbf{e}_{2} \otimes \mathbf{e}_{1}=\left[\begin{array}{ll}0 & 0 \\ 1 & 0\end{array}\right]$, $\chi^{(12)}=\mathbf{E}_{12}=\mathbf{e}_{1} \otimes \mathbf{e}_{2}=\left[\begin{array}{ll}0 & 1 \\ 0 & 0\end{array}\right]$ and $\chi^{(22)}=\mathbf{E}_{22}=\mathbf{e}_{2} \otimes \mathbf{e}_{2}=\left[\begin{array}{ll}0 & 0 \\ 0 & 1\end{array}\right]$. Breaking these down into their components via Equation 49 gives

$$
\begin{aligned}
& \chi_{2}^{(11)}=\chi_{3}^{(11)}=\chi_{4}^{(11)}=\mathbf{0} \text { and } \chi_{1}^{(11)}=\chi^{(11)}=\mathbf{E}_{11} \\
& \chi_{1}^{(21)}=\chi_{2}^{(21)}=\chi_{3}^{(21)}=\mathbf{0} \text { and } \chi_{4}^{(21)}=\chi^{(21)}=\mathbf{E}_{21} \\
& \chi_{1}^{(12)}=\chi_{2}^{(12)}=\chi_{3}^{(12)}=\mathbf{0} \text { and } \chi_{4}^{(12)}=\chi^{(12)}=\mathbf{E}_{12} \\
& \chi_{2}^{(22)}=\chi_{3}^{(22)}=\chi_{4}^{(22)}=\mathbf{0} \text { and } \chi_{1}^{(22)}=\chi^{(22)}=\mathbf{E}_{22}
\end{aligned}
$$

The null load cases and Equation 47 imply $\mathbf{w}^{(11)}=\mathbf{w}_{1}^{(11)}, \mathbf{w}^{(21)}=\mathbf{w}_{4}^{(21)}, \mathbf{w}^{(12)}=\mathbf{w}_{4}^{(12)}$, and $\mathbf{w}^{(22)}=\mathbf{w}_{1}^{(22)}$.

As in the scalar case, we must again take care to enforce periodic boundary conditions. For $\mathbf{x} \in \partial C_{x}=$ $\partial C \cap \Gamma_{x}$ we require that $\mathbf{w}\left(\mathbf{R}_{x} \mathbf{x}\right)=\mathbf{w}(\mathbf{x})$ and $\mathbf{t}\left(\mathbf{R}_{x} \mathbf{x}\right)=-\mathbf{t}(\mathbf{x})$; similarly for $\mathbf{y} \in \partial C_{y}=\partial C \cap \Gamma_{y}$ we require that $\mathbf{w}\left(\mathbf{R}_{y} \mathbf{y}\right)=\mathbf{w}(\mathbf{y})$ and $\mathbf{t}\left(\mathbf{R}_{y} \mathbf{y}\right)=-\mathbf{t}(\mathbf{y})$. For the first case with $\mathbf{x} \in \partial C_{x}$, periodicity and Equation 45 
require

$$
\begin{aligned}
\mathbf{w}_{i}(\mathbf{x}) & =\mathbf{w}_{i}\left(\mathbf{R}_{x} \mathbf{x}\right) \\
& =\rho_{i}\left(\sigma_{x}\right) \mathbf{R}_{x} \mathbf{w}_{i}(\mathbf{x}) \\
& =\rho_{i}\left(\sigma_{x}\right)\left\{\begin{array}{c}
w_{i_{x}}(\mathbf{x}) \\
-w_{i_{y}}(\mathbf{x})
\end{array}\right\}
\end{aligned}
$$

The above implies $w_{i_{y}}(\mathbf{x})=0$ for $i=1,2$ since $\rho_{1}\left(\sigma_{x}\right)=\rho_{2}\left(\sigma_{x}\right)=1$ and $w_{i_{x}}(\mathbf{x})=0$ for $i=3,4$ since $\rho_{3}\left(\sigma_{x}\right)=\rho_{4}\left(\sigma_{x}\right)=-1$.

The traction condition states that

$$
\begin{aligned}
\mathbf{t}_{i}(\mathbf{x}) & =-\mathbf{t}_{i}\left(\mathbf{R}_{x} \mathbf{x}\right) \\
& =-\rho_{i}\left(\sigma_{x}\right) \mathbf{R}_{x} \mathbf{t}_{i}(\mathbf{x}) \\
& =\rho_{i}\left(\sigma_{x}\right)\left\{\begin{array}{c}
-t_{i_{x}}(\mathbf{x}) \\
t_{i_{y}}(\mathbf{x})
\end{array}\right\}
\end{aligned}
$$

The computations leading to the above equality are similar with those in Equation 54 . We conclude that $t_{i_{x}}(\mathbf{x})=0$ for $i=1,2$ since $\rho_{1}\left(\sigma_{x}\right)=\rho_{2}\left(\sigma_{x}\right)=1$ and $t_{i_{y}}(\mathbf{x})=0$ for $i=3,4$ since $\rho_{3}\left(\sigma_{x}\right)=\rho_{4}\left(\sigma_{x}\right)=-1$.

Similarly, for $\mathbf{y} \in \partial C_{y}$, we have

$$
\begin{array}{r}
\mathbf{w}_{i}(\mathbf{y})=\mathbf{w}_{i}\left(\mathbf{R}_{y} \mathbf{y}\right)=\rho_{i}\left(\sigma_{y}\right) \mathbf{R}_{y} \mathbf{w}_{i}(\mathbf{y})=\rho_{i}\left(\sigma_{y}\right)\left\{\begin{array}{c}
-w_{i_{x}}(\mathbf{x}) \\
w_{i_{y}}(\mathbf{x})
\end{array}\right\} \\
\mathbf{t}_{i}(\mathbf{y})=-\mathbf{t}_{i}\left(\mathbf{R}_{y} \mathbf{y}\right)=-\rho_{i}\left(\sigma_{y}\right) \mathbf{R}_{y} \mathbf{t}_{i}(\mathbf{y})=\rho_{i}\left(\sigma_{y}\right)\left\{\begin{array}{c}
t_{i_{x}}(\mathbf{x}) \\
-t_{i_{y}}(\mathbf{x})
\end{array}\right\}
\end{array}
$$

In this case we must enforce $w_{i_{x}}(\mathbf{y})=0$ and $t_{i_{y}}(\mathbf{y})=0$ for $i=1,3$ since $\rho_{1}\left(\sigma_{y}\right)=\rho_{3}\left(\sigma_{y}\right)=1$; on the other hand, we must enforce $w_{i_{y}}(\mathbf{y})=0$ and $t_{i_{x}}(\mathbf{y})=0$ for $i=2,4$ since $\rho_{2}\left(\sigma_{y}\right)=\rho_{4}\left(\sigma_{y}\right)=-1$.

The four unit cell problems on the large domain $\Omega$ are thusly reduced to four unit cell problems on the symmetry cell $C$ which do not require the enforcement of periodicity. The first problem is to find $\mathbf{w}^{(11)}=\mathbf{w}_{1}^{(11)}$ such that

$$
\begin{aligned}
& \operatorname{div}\left(\mathbb{C}\left(\nabla \mathbf{w}^{(11)}-\mathbf{E}_{11}\right)=\mathbf{0} \text { in } C\right. \\
& \left\{\begin{array}{c}
w_{y}^{(11)} \\
t_{x}^{(11)}
\end{array}\right\}=\mathbf{0} \quad \text { on } \Sigma_{x} \cup \partial C_{x} \\
& \left\{\begin{array}{c}
w_{x}^{(11)} \\
t_{y}^{(11)}
\end{array}\right\}=\mathbf{0} \quad \text { on } \Sigma_{y} \cup \partial C_{y}
\end{aligned}
$$

the second problem is to find $\mathbf{w}^{(21)}=\mathbf{w}_{4}^{(21)}$ such that

$$
\begin{aligned}
& \operatorname{div}\left(\mathbb{C}\left(\nabla \mathbf{w}^{(21)}-\mathbf{E}_{21}\right)=\mathbf{0} \text { in } C\right. \\
& \left\{\begin{array}{c}
w_{x}^{(21)} \\
t_{y}^{(21)}
\end{array}\right\}=\mathbf{0} \quad \text { on } \Sigma_{x} \cup \partial C_{x} \\
& \left\{\begin{array}{c}
w_{y}^{(21)} \\
t_{x}^{(21)}
\end{array}\right\}=\mathbf{0} \quad \text { on } \Sigma_{y} \cup \partial C_{y}
\end{aligned}
$$

the third problem is to find $\mathbf{w}^{(12)}=\mathbf{w}_{4}^{(12)}$ such that

$$
\begin{aligned}
& \operatorname{div}\left(\mathbb{C}\left(\nabla \mathbf{w}^{(12)}-\mathbf{E}_{12}\right)\right.=\mathbf{0} \text { in } C \\
&\left\{\begin{array}{c}
w_{x}^{(12)} \\
t_{y}^{(12)}
\end{array}\right\}=\mathbf{0} \quad \text { on } \Sigma_{x} \cup \partial C_{x} \\
&\left\{\begin{array}{c}
w_{y}^{(12)} \\
t_{x}^{(12)}
\end{array}\right\}=\mathbf{0} \quad \text { on } \Sigma_{y} \cup \partial C_{y}
\end{aligned}
$$


and finally the fourth problem is to find $\mathbf{w}^{(22)}=\mathbf{w}_{1}^{(22)}$ such that

$$
\begin{aligned}
& \operatorname{div}\left(\mathbb{C}\left(\nabla \mathbf{w}(22)-\mathbf{E}_{22}\right)\right.=\mathbf{0} \text { in } C \\
&\left\{\begin{array}{c}
w_{y}^{(22)} \\
\left.t_{x}^{(22)}\right\}
\end{array}\right\} \mathbf{0} \text { on } \Sigma_{x} \cup \partial C_{x} \\
&\left\{\begin{array}{c}
w_{x}^{(22)} \\
t_{y}^{(22)}
\end{array}\right\}=\mathbf{0} \text { on } \Sigma_{y} \cup \partial C_{y}
\end{aligned}
$$

The finite element approximations for $\mathbf{w}^{(i)}$ require the evaluations and factorizations of four stiffness matrices defined on the small symmetry cell $C$. That withstanding, the stiffness matrices for the $\mathbf{w}^{(11)}$ and $\mathbf{w}^{(22)}$ problems are identical as are the stiffness matrices for the $\mathbf{w}^{(21)}$ and $\mathbf{w}^{(12)}$ problems. The latter should come as no surprise, because $\mathbf{w}^{(21)}=\mathbf{w}^{(12)}$ due to the minor symmetry of $\mathbb{C}$, i.e. $\mathbb{C}\left[\mathbf{E}_{21}\right]=\mathbb{C}\left[\mathbf{E}_{12}\right]$. But, as described at the end of Section 2.3, it is possible to evaluate and factor a single stiffness matrix on the symmetry sector if we accommodate the $w_{j}^{(i)}=0$ boundary conditions via constraints.

The homogenized material tensor is evaluated with the help of Equation 50, the change of variable theorem, the domain symmetry of $\mathbb{C}$ and the equalities $\mathbf{w}^{(11)}=\mathbf{w}_{1}^{(11)}, \mathbf{w}^{(21)}=\mathbf{w}_{4}^{(21)}, \mathbf{w}^{(12)}=\mathbf{w}_{4}^{(12)}$, and $\mathbf{w}^{(22)}=\mathbf{w}_{1}^{(22)}$, i.e.

$$
\begin{aligned}
& \mathbb{C}_{h}=\frac{1}{\operatorname{vol}(\Omega)} \int_{\Omega} \mathbb{C}(\mathbf{x})\left(\mathbb{\square}+\nabla \mathbf{w}^{(11)}(\mathbf{x}) \otimes \mathbf{E}_{11}+\nabla \mathbf{w}^{(21)}(\mathbf{x}) \otimes \mathbf{E}_{21}+\nabla \mathbf{w}^{(12)}(\mathbf{x}) \otimes \mathbf{E}_{12}+\nabla \mathbf{w}^{(22)}(\mathbf{x}) \otimes \mathbf{E}_{22}\right) d a \\
& =\frac{1}{\operatorname{vol}(\Omega)} \int_{\Omega} \mathbb{C}(\mathbf{x})\left(\mathbb{\square}+\nabla \mathbf{w}_{1}^{(11)}(\mathbf{x}) \otimes \mathbf{E}_{11}+\nabla \mathbf{w}_{4}^{(21)}(\mathbf{x}) \otimes \mathbf{E}_{21}+\nabla \mathbf{w}_{4}^{(12)}(\mathbf{x}) \otimes \mathbf{E}_{12}+\nabla \mathbf{w}_{1}^{(22)}(\mathbf{x}) \otimes \mathbf{E}_{22}\right) d a \\
& =\sum_{g} \frac{1}{\operatorname{vol}(\Omega)} \int_{\Omega} \mathbb{C}\left(\mathbf{R}_{g} \mathbf{x}\right)\left(\mathbb{\square}+\nabla \mathbf{w}_{1}^{(11)}\left(\mathbf{R}_{g} \mathbf{x}\right) \otimes \mathbf{E}_{11}+\right. \\
& \left.\nabla \mathbf{w}_{4}^{(21)}\left(\mathbf{R}_{g} \mathbf{x}\right) \otimes \mathbf{E}_{21}+\nabla \mathbf{w}_{4}^{(12)}\left(\mathbf{R}_{g} \mathbf{x}\right) \otimes \mathbf{E}_{12}+\nabla \mathbf{w}_{1}^{(22)}\left(\mathbf{R}_{g} \mathbf{x}\right) \otimes \mathbf{E}_{22}\right) d a \\
& =\sum_{g} \frac{1}{\operatorname{vol}(\Omega)} \int_{\Omega}\left(\mathbf{R}_{g} \otimes \mathbf{R}_{g}\right) \mathbb{C}(\mathbf{x})\left(\mathbf{R}_{g} \otimes \mathbf{R}_{g}\right)^{-1}\left(\mathbb{\square}+\left(\mathbf{R}_{g} \otimes \mathbf{R}_{g}\right)\left[\rho_{1}\left(\sigma_{g}\right) \nabla \mathbf{w}_{1}^{(11)}(\mathbf{x}) \otimes \mathbf{E}_{11}+\right.\right. \\
& \left.\rho_{4}\left(\sigma_{g}\right) \nabla \mathbf{w}_{4}^{(21)}(\mathbf{x}) \otimes \mathbf{E}_{21}+\rho_{4}\left(\sigma_{g}\right) \nabla \mathbf{w}_{4}^{(12)}(\mathbf{x}) \otimes \mathbf{E}_{12}+\rho_{1}\left(\sigma_{g}\right) \nabla \mathbf{w}_{1}^{(22)}(\mathbf{x}) \otimes \mathbf{E}_{22}\right) d a \\
& =\sum_{g} \frac{1}{\operatorname{vol}(\Omega)} \int_{C}\left(\mathbf{R}_{g} \otimes \mathbf{R}_{g}\right) \mathbb{C}\left(\left(\mathbf{R}_{g} \otimes \mathbf{R}_{g}\right)^{-1}+\rho_{1}\left(\sigma_{g}\right) \nabla \mathbf{w}_{1}^{(11)}(\mathbf{x}) \otimes \mathbf{E}_{11}+\right. \\
& \left.\rho_{4}\left(\sigma_{g}\right) \nabla \mathbf{w}_{4}^{(21)}(\mathbf{x}) \otimes \mathbf{E}_{21}+\rho_{4}\left(\sigma_{g}\right) \nabla \mathbf{w}_{4}^{(12)}(\mathbf{x}) \otimes \mathbf{E}_{12}+\rho_{1}\left(\sigma_{g}\right) \nabla \mathbf{w}_{1}^{(22)}(\mathbf{x}) \otimes \mathbf{E}_{22}\right) d a
\end{aligned}
$$

where we used the result that $(\mathbf{A C B}) \otimes \mathbf{D}=\left(\mathbf{A} \otimes \mathbf{B}^{T}\right)(\mathbf{C} \otimes \mathbf{D})$ for 2-tensors $\mathbf{A}, \mathbf{B}, \mathbf{C}$ and $\mathbf{D}$. Indeed, for any 2-tensor $\mathbf{E}$ we have $((\mathbf{A C B}) \otimes \mathbf{D})[\mathbf{E}]=(\mathbf{D} \cdot \mathbf{E}) \mathbf{A C B}=\left(\mathbf{A} \otimes \mathbf{B}^{T}\right)[(\mathbf{D} \cdot \mathbf{E}) \mathbf{C}]=\left(\mathbf{A} \otimes \mathbf{B}^{T}\right)(\mathbf{C} \otimes \mathbf{D})[\mathbf{E}]$.

\subsection{Nonabelian symmetry}

For nonabelian groups we construct the projectors such that for any vector-valued function on the symmetry domain $\mathbf{w}: \Omega \rightarrow \mathcal{R}^{2}$ we have

$$
P_{i}^{j k} \mathbf{w}(\mathbf{x})=\frac{d_{i}}{n} \sum_{g \in \mathcal{G}} \rho_{i}^{j k}\left(\sigma_{g}\right) \mathbf{R}_{g} \mathbf{w}\left(\mathbf{R}_{g}^{-1} \mathbf{x}\right)
$$


Following the development of Equation 44 we have

$$
\begin{aligned}
& P_{i}^{j k} \mathbf{w}\left(\mathbf{R}_{h}^{-1} \mathbf{x}\right)=\frac{d_{i}}{n} \sum_{g \in \mathcal{G}} \rho_{i}^{j k}\left(\sigma_{g}\right) \mathbf{R}_{g} \mathbf{w}\left(\mathbf{R}_{g}^{-1}\left(\mathbf{R}_{h}^{-1} \mathbf{x}\right)\right) \\
& =\frac{d_{i}}{n} \sum_{g \in \mathcal{G}} \sum_{s=1}^{d_{i}} \overbrace{\rho_{i}^{j s}\left(\sigma_{h}^{-1} \sigma_{h}\right)}^{=\delta_{j s}} \rho_{i}^{s k}\left(\sigma_{g}\right) \overbrace{\mathbf{R}_{h}^{-1} \mathbf{R}_{h}}^{=\mathbf{I}} \mathbf{R}_{g} \mathbf{w}\left(\left(\mathbf{R}_{h} \mathbf{R}_{g}\right)^{-1} \mathbf{x}\right) \\
& =\frac{d_{i}}{n} \sum_{g \in \mathcal{G}} \sum_{r=1}^{d_{i}} \sum_{s=1}^{d_{i}} \rho_{i}^{j r}\left(\sigma_{h}^{-1}\right) \rho_{i}^{r s}\left(\sigma_{h}\right) \rho_{i}^{s k}\left(\sigma_{g}\right) \mathbf{R}_{h}^{-1}\left(\mathbf{R}_{h} \mathbf{R}_{g}\right) \mathbf{w}\left(\left(\mathbf{R}_{h} \mathbf{R}_{g}\right)^{-1} \mathbf{x}\right) \\
& =\frac{d_{i}}{n} \sum_{g \in \mathcal{G}} \sum_{r=1}^{d_{i}} \rho_{i}^{r j}\left(\sigma_{h}\right) \rho_{i}^{r k}\left(\sigma_{h} \sigma_{g}\right) \mathbf{R}_{h}^{-1}\left(\mathbf{R}_{h} \mathbf{R}_{g}\right) \mathbf{w}\left(\left(\mathbf{R}_{h} \mathbf{R}_{g}\right)^{-1} \mathbf{x}\right) \\
& =\sum_{r=1}^{d_{i}} \rho_{i}^{r j}\left(\sigma_{h}\right) \mathbf{R}_{h}^{-1} \frac{d_{i}}{n} \sum_{g \in \mathcal{G}} \rho_{i}^{r k}\left(\sigma_{h} \sigma_{g}\right)\left(\mathbf{R}_{h} \mathbf{R}_{g}\right) \mathbf{w}\left(\left(\mathbf{R}_{h} \mathbf{R}_{g}\right)^{-1} \mathbf{x}\right) \\
& =\sum_{r=1}^{d_{i}} \rho_{i}^{r j}\left(\sigma_{h}\right) \mathbf{R}_{h}^{-1} P_{i}^{r k} \mathbf{w}(\mathbf{x})
\end{aligned}
$$

Using the orthogonality of the irreps and manipulating the above also gives

$$
\begin{aligned}
P_{i}^{j k} \mathbf{w}\left(\mathbf{R}_{h} \mathbf{x}\right) & =\sum_{r=1}^{d_{i}} \rho_{i}^{r j}\left(\sigma_{h}^{-1}\right) \mathbf{R}_{h} P_{i}^{r k} \mathbf{w}(\mathbf{x}) \\
& =\sum_{r=1}^{d_{i}} \rho_{i}^{j r}\left(\sigma_{h}\right) \mathbf{R}_{h} P_{i}^{r k} \mathbf{w}(\mathbf{x})
\end{aligned}
$$

which can be recast as matrix equation, cf. Equation 45

$$
\mathbf{P}_{i}^{k} \mathbf{w}\left(\mathbf{R}_{h} \mathbf{x}\right)=\left(\boldsymbol{\rho}_{i}\left(\sigma_{h}\right) \odot \mathbf{R}_{h}\right) \mathbf{P}_{i}^{k} \mathbf{w}(\mathbf{x})
$$

where $\mathbf{P}_{i}^{k} \mathbf{w}=\left[\begin{array}{llll}P_{i}^{1 k} \mathbf{w}^{T} & P_{i}^{2 k} \mathbf{w}^{T} & \ldots & P_{i}^{d_{i} k} \mathbf{w}^{T}\end{array}\right]^{T}$ is the $i-k$ family which again plays an important role in the subsequent analysis.

And following the development of Equation 46 we find

$$
\begin{aligned}
P_{i}^{j k} P_{r}^{s t} \mathbf{w}(\mathbf{x}) & =\frac{d_{i}}{n} \sum_{g \in \mathcal{G}} \rho_{i}^{j k}\left(\sigma_{g}\right) \mathbf{R}_{g} P_{r}^{s t} \mathbf{w}\left(\mathbf{R}_{g}^{-1} \mathbf{x}\right) \\
& =\frac{d_{i}}{n} \sum_{g \in \mathcal{G}} \rho_{i}^{j k}\left(\sigma_{g}\right) \mathbf{R}_{g} \mathbf{R}_{g}^{-1} \sum_{u=1}^{d_{r}} \rho_{r}^{u s}\left(\sigma_{g}\right) P_{r}^{u t} \mathbf{w}(\mathbf{x}) \\
& =\frac{d_{i}}{n} \sum_{u=1}^{d_{r}} P_{r}^{u t} \mathbf{w}(\mathbf{x}) \sum_{g \in \mathcal{G}} \rho_{i}^{j k}\left(\sigma_{g}\right) \rho_{r}^{u s}\left(\sigma_{g}\right) \\
& =\frac{d_{i}}{n} \sum_{u=1}^{d_{r}} P_{r}^{u t} \mathbf{w}(\mathbf{x}) \delta_{i r} \delta_{j u} \delta_{k s} \frac{n}{d_{i}} \\
& =\delta_{i r} \delta_{k s} P_{r}^{j t} \mathbf{w}(\mathbf{x})
\end{aligned}
$$

where we again utilized the row wise orthogonality of the irreps.

Slightly different than Equation 47, but inline with Equation 25, for the nonabelian case we have

$$
\mathbf{w}(\mathbf{x})=\sum_{i=1}^{n_{\rho}} \sum_{j=1}^{d_{i}} P_{i}^{j j} \mathbf{w}(\mathbf{x}) .
$$


The proof is analogous to that of Equation 26. So as discussed so many times before, we again partition $\mathcal{H}$ into complementary subspaces, i.e. $\mathcal{H}(\Omega)=\bigoplus_{i=1}^{n_{\rho}} \bigoplus_{j=1}^{d_{i}} P_{i}^{j j} \mathcal{H}(\Omega)$. Again, the mixed terms e.g. $P_{3}^{12}$, do not appear in the expansion. However, as in the scalar case, they are of vital importance.

Following the developments of Equations 48-50 and 57-59 we note that

$$
\begin{aligned}
\nabla P_{i}^{j k} \mathbf{w}\left(\mathbf{R}_{h}^{-1} \mathbf{x}\right) & =\sum_{r=1}^{d_{i}} \rho_{i}^{r j}\left(\sigma_{h}\right) \mathbf{R}_{h}^{-1} \nabla P_{i}^{r k} \mathbf{w}(\mathbf{x}) \mathbf{R}_{h} \\
\nabla P_{i}^{j k} \mathbf{w}\left(\mathbf{R}_{h} \mathbf{x}\right) & =\sum_{r=1}^{d_{i}} \rho_{i}^{j r}\left(\sigma_{h}\right) \mathbf{R}_{h} \nabla P_{i}^{r k} \mathbf{w}(\mathbf{x}) \mathbf{R}_{h}^{-1}
\end{aligned}
$$

as well as

$$
\begin{aligned}
P_{i}^{j k} \chi(\mathbf{x}) & =\frac{d_{i}}{n} \sum_{g \in \mathcal{G}} \rho_{i}^{j k}\left(\sigma_{g}\right) \mathbf{R}_{g} \chi\left(\mathbf{R}_{g}^{-1} \mathbf{x}\right) \mathbf{R}_{g}^{-1} \\
P_{i}^{j k} \boldsymbol{\chi}\left(\mathbf{R}_{h}^{-1} \mathbf{x}\right) & =\sum_{r=1}^{d_{i}} \rho_{i}^{r j}\left(\sigma_{h}\right) \mathbf{R}_{h}^{-1} P_{i}^{r k} \boldsymbol{\chi}(\mathbf{x}) \mathbf{R}_{h} \\
P_{i}^{j k} \chi\left(\mathbf{R}_{h} \mathbf{x}\right) & =\sum_{r=1}^{d_{i}} \rho_{i}^{j r}\left(\sigma_{h}\right) \mathbf{R}_{h} P_{i}^{r k} \chi(\mathbf{x}) \mathbf{R}_{h}^{-1}
\end{aligned}
$$

We return to the integral Equation 42 and follow the derivation of Equation 51. Here we substitute $\mathbf{v}_{i}^{j j}=P_{i}^{j j} \mathbf{v}$ into Equation 42 which gives

$$
\begin{aligned}
& \int_{\Omega} \nabla \mathbf{v}_{i}^{j j}(\mathbf{x}) \cdot \mathbb{C}(\mathbf{x})[\nabla w(\mathbf{x})] d v=\int_{\Omega}\left(\nabla \mathbf{v}_{i}^{j j}(\mathbf{x}) \cdot \mathbb{C}(\mathbf{x})[\boldsymbol{\chi}(\mathbf{x})]+\mathbf{v}_{i}^{j j}(\mathbf{x}) \cdot \mathbf{f}(\mathbf{x})\right) d v+ \\
& \int_{\partial \Omega^{n}} \mathbf{v}_{i}^{j j}(\mathbf{x}) \cdot \mathbf{t}^{p}(\mathbf{x}) d a \\
& \sum_{g \in \mathcal{G}} \int_{\mathbf{R}_{g}^{-1}(C)} \nabla \mathbf{v}_{i}^{j j}(\mathbf{x}) \cdot \mathbb{C}(\mathbf{x})[\nabla w(\mathbf{x})] d v=\sum_{g \in \mathcal{G}} \int_{\mathbf{R}_{g}^{-1}(C)}\left(\nabla \mathbf{v}_{i}^{j j}(\mathbf{x}) \cdot \mathbb{C}(\mathbf{x})[\boldsymbol{\chi}(\mathbf{x})]+\mathbf{v}_{i}^{j j}(\mathbf{x}) \cdot \mathbf{f}(\mathbf{x})\right) d v+ \\
& \sum_{g \in \mathcal{G}} \int_{\mathbf{R}_{g}^{-1}\left(\partial C^{n}\right)} \mathbf{v}_{i}^{j j}(\mathbf{x}) \cdot \mathbf{t}^{p}(\mathbf{x}) d a \\
& \sum_{g \in \mathcal{G}} \int_{C} \nabla \mathbf{v}_{i}^{j j}\left(\mathbf{R}_{g}^{-1} \mathbf{x}\right) \cdot \mathbb{C}\left(\mathbf{R}_{g}^{-1} \mathbf{x}\right)\left[\nabla w\left(\mathbf{R}_{g}^{-1} \mathbf{x}\right)\right] d v=\sum_{g \in \mathcal{G}} \int_{C}\left(\nabla \mathbf{v}_{i}^{j j}\left(\mathbf{R}_{g}^{-1} \mathbf{x}\right) \cdot \mathbb{C}\left(\mathbf{R}_{g}^{-1} \mathbf{x}\right)\left[\boldsymbol{\chi}\left(\mathbf{R}_{g}^{-1} \mathbf{x}\right)\right]+\right. \\
& \left.\mathbf{v}_{i}^{j j}\left(\mathbf{R}_{g}^{-1} \mathbf{x}\right) \cdot \mathbf{f}\left(\mathbf{R}_{g}^{-1} \mathbf{x}\right)\right) d v+ \\
& \sum_{g \in \mathcal{G}} \int_{\partial C^{n}} \mathbf{v}_{i}^{j j}\left(\mathbf{R}_{g}^{-1} \mathbf{x}\right) \cdot \mathbf{t}^{p}\left(\mathbf{R}_{g}^{-1} \mathbf{x}\right) d a \\
& \sum_{g \in \mathcal{G}} \sum_{l=1}^{d_{i}} \int_{C} \rho_{i}^{l j}\left(\sigma_{g}\right) \mathbf{R}_{g}^{-1} \nabla \mathbf{v}_{i}^{l j}(\mathbf{x}) \mathbf{R}_{g} \cdot\left(\mathbf{R}_{g} \otimes \mathbf{R}_{g}\right)^{-1} \mathbb{C}(\mathbf{x})\left(\mathbf{R}_{g} \otimes \mathbf{R}_{g}\right)\left[\nabla w\left(\mathbf{R}_{g}^{-1} \mathbf{x}\right)\right] d v=\sum_{g \in \mathcal{G}} \sum_{l=1}^{d_{i}} \int_{C}\left(\rho_{i}^{l j}\left(\sigma_{g}\right) \mathbf{R}_{g}^{-1} \nabla \mathbf{v}_{i}^{l j}(\mathbf{x}) \mathbf{R}_{g}\right. \\
& \cdot\left(\mathbf{R}_{g} \otimes \mathbf{R}_{g}\right)^{-1} \mathbb{C}(\mathbf{x})\left(\mathbf{R}_{g} \otimes \mathbf{R}_{g}\right)\left[\boldsymbol{\chi}\left(\mathbf{R}_{g}^{-1} \mathbf{x}\right)\right]+ \\
& \left.\rho_{i}^{l j}\left(\sigma_{g}\right) \mathbf{R}_{g}^{-1} \mathbf{v}_{i}^{l j}(\mathbf{x}) \cdot \mathbf{f}\left(\mathbf{R}_{g}^{-1} \mathbf{x}\right)\right) d v+ \\
& \sum_{g \in \mathcal{G}} \sum_{l=1}^{d_{i}} \int_{\partial C^{n}} \rho_{i}^{l j}\left(\sigma_{g}\right) \mathbf{R}_{g}^{-1} \mathbf{v}_{i}^{l j}(\mathbf{x}) \cdot \mathbf{t}^{p}\left(\mathbf{R}_{g}^{-1} \mathbf{x}\right) d a \\
& \sum_{g \in \mathcal{G}} \sum_{l=1}^{d_{i}} \int_{C} \nabla \mathbf{v}_{i}^{l j}(\mathbf{x}) \cdot \mathbb{C}(\mathbf{x})\left[\rho_{i}^{l j}\left(\sigma_{g}\right) \mathbf{R}_{g} \nabla w\left(\mathbf{R}_{g}^{-1} \mathbf{x}\right) \mathbf{R}_{g}^{-1}\right] d v=\sum_{g \in \mathcal{G}} \sum_{l=1}^{d_{i}} \int_{C}\left(\nabla \mathbf{v}_{i}^{l j}(\mathbf{x}) \cdot \mathbb{C}(\mathbf{x})\left[\rho_{i}^{l j}\left(\sigma_{g}\right) \mathbf{R}_{g} \boldsymbol{\chi}\left(\mathbf{R}_{g}^{-1} \mathbf{x}\right) \mathbf{R}_{g}^{-1}\right]+\right. \\
& \left.\mathbf{v}_{i}^{l j}(\mathbf{x}) \cdot \rho_{i}^{l j}\left(\sigma_{g}\right) \mathbf{R}_{g} \mathbf{f}\left(\mathbf{R}_{g}^{-1} \mathbf{x}\right)\right) d v+ \\
& \sum_{g \in \mathcal{G}} \sum_{l=1}^{d_{i}} \int_{\partial C^{n}} \mathbf{v}_{i}^{l j}(\mathbf{x}) \cdot \mathbf{R}_{g} \rho_{i}^{l j}\left(\sigma_{g}\right) \mathbf{t}^{p}\left(\mathbf{R}_{g}^{-1} \mathbf{x}\right) d a \\
& \frac{n}{d_{i}} \sum_{l=1}^{d_{i}} \int_{C} \nabla \mathbf{v}_{i}^{l j}(\mathbf{x}) \cdot \mathbb{C}(\mathbf{x}) \nabla \mathbf{w}_{i}^{l j}(\mathbf{x}) d v=\frac{n}{d_{i}} \sum_{l=1}^{d_{i}} \int_{C}\left(\nabla \mathbf{v}_{i}^{l j}(\mathbf{x}) \cdot \mathbb{C}(\mathbf{x}) \boldsymbol{\chi}_{i}^{l j} \mathbf{x}\right)+ \\
& \left.\mathbf{v}_{i}^{l j}(\mathbf{x}) \mathbf{f}_{i}^{l j}(\mathbf{x})\right) d v+ \\
& \frac{n}{d_{i}} \sum_{l=1}^{d_{i}} \int_{\partial C n} \mathbf{v}_{i}^{l j}(\mathbf{x}) \mathbf{t}_{i}^{p^{l j}}(\mathbf{x}) d a
\end{aligned}
$$


where it is understood that, e.g. $\mathbf{w}_{i}^{l j}=P_{i}^{l j} \mathbf{w}$, etc. To obtain the above we used the summation of integration, change-of-variable theorem, Equations $56-57$ and the domain symmetry of $\mathbb{C}$.

Now we follow the development of Equation 52 wherein we use Equation 60 to express $\mathbf{v}=\sum_{i=1}^{n_{\rho}} \sum_{j=1}^{d_{i}} \mathbf{v}_{i}^{j j}$ and substitute into Equation 42. Each $\mathbf{v}_{i}^{j j}$ renders an integral expression which only contains $\mathbf{w}_{i}^{l j}$ for $l=1,2, \ldots, d_{i}$. We evaluate the response $\mathbf{w}_{i}^{l j}$ by setting $\mathbf{v}_{i}^{m j}=\mathbf{0}$ for $m \neq l$. In light of this the boundary value problem of Equation 42 defined on $\Omega$ is now converted into $n=\sum_{i=1}^{n_{\rho}} d_{i}^{2}$ problems defined on $C$ which read: find the response $\mathbf{w}_{i}^{l j} \in \mathcal{H}\left(C, \mathbf{w}_{i}^{p^{l j}}\right)$ such that

$$
\int_{C} \nabla \mathbf{v}_{i}^{l j} \cdot \mathbb{C} \nabla \mathbf{w}_{i}^{l j} d v=\int_{C}\left(\nabla v_{i}^{l j} \cdot \mathbb{C} \chi_{i}^{l j}+\mathbf{v}_{i}^{l j} \mathbf{f}_{i}^{l j}\right) d v+\int_{\partial C^{n}} \mathbf{v}_{i}^{l j} \mathbf{t}_{i}^{p^{l j}} d a
$$

for all $\mathbf{v}_{i}^{l j} \in \mathcal{H}(C, \mathbf{0})$. Again the benefit these problems offer is their replacement of one problem defined over the large domain $\Omega$ by $n$ problems defined over the smaller symmetry cell $C$.

As before, the above result is not satisfactory because we have not incorporated the boundary conditions on the cut surfaces of $C$. For example, referring to the $D_{3}$ symmetric domain of Figure 4, a point $\mathbf{x} \in C$ is on $\Sigma_{y 2} \subset \Sigma$ if $\mathbf{x}=\mathbf{R}_{y 2} \mathbf{x}$; and it is on $\Sigma_{y} \subset \Sigma$ if $\mathbf{x}=\mathbf{R}_{y} \mathbf{x}$. Again, we allow neither jumps in the response nor in the flux throughout the domain, and thus the $\mathbf{w}_{i}^{l r}$ for $i=1, \ldots, n_{\rho}$ and $l, r=1, \ldots, d_{i}$ at each of these points must satisfy

$$
\begin{aligned}
\llbracket \mathbf{w}_{i}^{l r}(\mathbf{x}) \rrbracket & =\mathbf{0} \\
\llbracket \mathbb{C}(\mathbf{x})\left[\nabla \mathbf{w}_{i}^{l r}(\mathbf{x})-\chi_{i}^{l r}(\mathbf{x})\right] \rrbracket \mathbf{n}(\mathbf{x}) & =\mathbf{0}
\end{aligned}
$$

Considering a general cut boundary point $\mathbf{x}=\mathbf{R}_{g} \mathbf{x} \in \Sigma_{g}$, substituting Equation 58 reduces the first jump condition to

$$
\begin{aligned}
\mathbf{w}_{i}^{l r}(\mathbf{x}) & =\mathbf{w}_{i}^{l r}\left(\mathbf{R}_{g} \mathbf{x}\right) \\
& =\sum_{j=1}^{d_{i}} \rho_{i}^{l j}\left(\sigma_{g}\right) \mathbf{R}_{g} \mathbf{w}_{i}^{j r}(\mathbf{x})
\end{aligned}
$$

which can alternately be expressed as

$$
\frac{1}{2}\left(\mathbf{I}-\left(\boldsymbol{\rho}_{i}\left(\sigma_{h}\right) \odot \mathbf{R}_{h}\right)\right) \mathbf{w}_{i}^{r}(\mathbf{x})=\mathbf{0}
$$

where $\mathbf{w}_{i}^{r}=\left[\begin{array}{llll}\mathbf{w}_{i}^{1 r^{T}} & \mathbf{w}_{i}^{2 r^{T}} & \ldots & \mathbf{w}_{i}^{d_{i} r^{T}}\end{array}\right]^{T}$ consolidates the $i-r$ family of functions and $\mathbf{I}$ is the $2 d_{i} \times 2 d_{i}$ identity, cf. Equation 55. These $r=1,2, \ldots, 2 d_{i}$ constraint equations must be satisfied and hence we see that the problems of Equation 52 involving the family $\mathbf{w}_{i}^{r}$ are coupled. Again, the role the mixed terms, e.g. $\mathbf{w}_{3}^{21}$ is illuminated here. Indeed, they are needed to compute $\mathbf{w}_{i}^{r r}$ which are used in Equation 60 to compute w. Also of note here is the form of Equation 64 wherein we recognize that $\left(1, \mathbf{w}_{i}^{r}(\mathbf{x})\right)$ is an eigenpair of the orthogonal matrix $\left(\boldsymbol{\rho}_{i}\left(\sigma_{h}\right) \odot \mathbf{R}_{h}\right)$ assuming $\mathbf{w}_{i}^{r}(\mathbf{x})$ is real and nonzero. Thusly, the number of independent constraint equations that are generated at each point $\mathbf{x} \in \Sigma_{g}$ from Equation 64 is less than $2 d_{i}$, depending on the multiplicity of the $\lambda=1$ eigenvalue.

As in the abelian case, we need not concern ourselves with the flux jump condition because the resulting homogeneous natural boundary conditions are satisfied defacto in the weak formulation. Nonetheless, for completeness we derive them by substituting Equations 61 and 62 to reduce the second jump Equation 63 
to

$$
\begin{aligned}
\mathbb{C}(\mathbf{x})\left[\nabla \mathbf{w}_{i}^{l r}(\mathbf{x})-\chi_{i}^{l r}(\mathbf{x})\right] \mathbf{n}(\mathbf{x}) & =\mathbb{C}\left(\mathbf{R}_{g} \mathbf{x}\right)\left[\nabla \mathbf{w}_{i}^{l r}\left(\mathbf{R}_{g} \mathbf{x}\right)-\boldsymbol{\chi}_{i}^{l r}\left(\mathbf{R}_{g} \mathbf{x}\right)\right] \mathbf{n}(\mathbf{x}) \\
& =\sum_{j=1}^{d_{i}} \rho_{i}^{l j}\left(\sigma_{g}\right)\left(\mathbf{R}_{g} \otimes \mathbf{R}_{g}\right) \mathbb{C}(\mathbf{x})\left(\mathbf{R}_{g} \otimes \mathbf{R}_{g}\right)^{-1}\left[\mathbf{R}_{g}\left(\nabla \mathbf{w}_{i}^{j r}(\mathbf{x})-\chi_{i}^{j r}(\mathbf{x})\right) \mathbf{R}_{g}^{-1}\right] \mathbf{n}(\mathbf{x}) \\
& =\sum_{r=1}^{d_{i}} \rho_{i}^{l j}\left(\sigma_{g}\right) \mathbf{R}_{g} \mathbb{C}(\mathbf{x})\left[\nabla \mathbf{w}_{i}^{j r}(\mathbf{x})-\chi_{i}^{j r}(\mathbf{x})\right] \mathbf{R}_{g}^{T} \mathbf{n}(\mathbf{x}) \\
& =-\mathbf{R}_{g} \mathbb{C}(\mathbf{x})\left[\sum_{r=1}^{d_{i}} \rho_{i}^{l j}\left(\sigma_{g}\right)\left(\nabla \mathbf{w}_{i}^{j r}(\mathbf{x})-\chi_{i}^{j r}(\mathbf{x})\right)\right] \mathbf{n}(\mathbf{x}) \\
& =-\mathbf{R}_{g} \sum_{r=1}^{d_{i}} \rho_{i}^{l j}\left(\sigma_{g}\right) \mathbf{t}_{i}^{j r}(\mathbf{x})
\end{aligned}
$$

where we use the fact that $\mathbf{R}_{g}^{T} \mathbf{n}(\mathbf{x})=-\mathbf{n}(\mathbf{x})$ for $\mathbf{x} \in \Sigma_{g}$ and recall the traction definition $\mathbf{t}_{i}^{j r}=\mathbb{C}\left[\nabla \mathbf{w}_{i}^{j r}-\right.$ $\left.\boldsymbol{\chi}_{i}^{j r}\right] \mathbf{n}$. In terms of the family of tractions $\mathbf{t}_{i}^{r}=\left[\begin{array}{llll}\mathbf{t}_{i}^{1 r^{T}} & \mathbf{t}_{i}^{2 r^{T}} & \ldots & \mathbf{t}_{i}^{d_{i} r^{T}}\end{array}\right]^{T}$ we equivalently have

$$
\frac{1}{2}\left(\mathbf{I}+\left(\boldsymbol{\rho}_{i}\left(\sigma_{h}\right) \odot \mathbf{R}_{h}\right)\right) \mathbf{t}_{i}^{r}(\mathbf{x})=\mathbf{0}
$$

cf. Equation 55. Again, referring to the discussion after Equation 18, here we see that $1 / 2\left(\mathbf{I}-\left(\boldsymbol{\rho}_{i}\left(\sigma_{h}\right) \odot \mathbf{R}_{h}\right)\right)$ and $1 / 2\left(\mathbf{I}+\left(\boldsymbol{\rho}_{i}\left(\sigma_{h}\right) \odot \mathbf{R}_{h}\right)\right)$ are complementary projections. And as per the conversation after Equation 64 , we see that $\left(-1, \mathbf{t}_{i}^{l}(\mathbf{x})\right)$ is an eigenpair of $\left(\boldsymbol{\rho}_{i}\left(\sigma_{h}\right) \odot \mathbf{R}_{h}\right)$, assuming $\mathbf{t}_{i}^{l}(\mathbf{x})$ is real and nonzero; hence, less than $d_{i}$ independent flux constraint equations are generated from Equation 32.

Once the responses $\mathbf{w}_{i}^{j k}$ on $C$ are computed they are used to evaluate $\mathbf{w}_{i}$ throughout $\Omega$ via Equation 58 and summed via Equation 60 to evaluate w, i.e.

$$
\mathbf{w}\left(\mathbf{R}_{g} \mathbf{x}\right)=\sum_{i=1}^{n_{\rho}} \sum_{j=1}^{d_{i}} \mathbf{w}_{i}^{j j}\left(\mathbf{R}_{g} \mathbf{x}\right)=\sum_{i=1}^{n_{\rho}} \sum_{j=1}^{d_{i}} \sum_{k=1}^{d_{i}} \rho_{i}^{j k}\left(\sigma_{g}\right) \mathbf{w}_{i}^{k j}(\mathbf{x})
$$

The role of the mixed terms, e.g. $\mathbf{w}_{3}^{21}$, is again seen.

For our $D_{3}$ example we have $d_{1}=1, d_{2}=1$ and $d_{3}=2$ and thusly there is no coupling for the $\mathbf{w}_{1}$ and $\mathbf{w}_{2}$ problems.

1. Concerning the $\mathbf{w}_{1}$ problem for $\mathbf{x} \in \Sigma_{y}$ Equations 64 and 65 give $\left(\mathbf{I}-\rho_{1}\left(\sigma_{y}\right) \mathbf{R}_{y}\right) \mathbf{w}_{1}=\left[\begin{array}{ll}w_{1_{x}} & 0\end{array}\right]^{T}=$ $\mathbf{0}$ and $\left(\mathbf{I}+\rho_{1}\left(\sigma_{y}\right) \mathbf{R}_{y}\right) \mathbf{t}_{1}=\left[\begin{array}{cc}0 & t_{1_{y}}\end{array}\right]^{T}=\mathbf{0}$. Note that the $\mathbf{R}_{y}$ eigenpairs $\left(-1,\left[\begin{array}{cc}1 & 0\end{array}\right]^{T}\right)$ and $\left(1,\left[\begin{array}{ll}0 & 1\end{array}\right]^{T}\right)$ are respectively perpendicular to any admissible $\mathbf{w}_{1}=\left[\begin{array}{ll}0 & w_{1}\end{array}\right]^{T}$ and $\mathbf{t}_{1}=\left[\begin{array}{cc}t_{1} & 0\end{array}\right]^{T}$ that satisfy the constraints, e.g. $\left[\begin{array}{ll}1 & 0\end{array}\right]^{T}\left[\begin{array}{ll}0 & w_{1}\end{array}\right]=0$. Performing similar calculations for $\mathbf{x} \in \Sigma_{y 2}$ gives $\left(\mathbf{I}-\rho_{1}\left(\sigma_{y 2}\right) \mathbf{R}_{y 2}\right) \mathbf{w}_{1}=1 / 4\left[\begin{array}{ll}w_{1_{x}}-\sqrt{3} w_{1_{y}}-\sqrt{3} w_{1_{x}}+3 w_{1_{y}}\end{array}\right]^{T}=\mathbf{0}$ and $\left(\mathbf{I}+\rho_{1}\left(\sigma_{y 2}\right) \mathbf{R}_{y 2}\right) \mathbf{t}_{1}=$ $1 / 4\left[\begin{array}{ll}3 t_{1_{x}}+\sqrt{3} t_{1_{y}} & \sqrt{3} t_{1_{x}}+t_{1_{y}}\end{array}\right]^{T}=\mathbf{0}$. Note that the same constraint equation appears twice for both $\mathbf{w}_{1}$ and $\mathbf{t}_{1}$ and that the admissible $\mathbf{w}_{1}=\left[\begin{array}{lll}\sqrt{3} w_{1} & w_{1}\end{array}\right]^{T}$ and $\mathbf{t}_{1}=\left[\begin{array}{ll}-t_{1} & \sqrt{3} t_{1}\end{array}\right]^{T}$ are respectively perpendicular to the $\mathbf{R}_{y 2}$ eigenpairs $\left(-1,\left[\begin{array}{ll}-1 & \sqrt{3}\end{array}\right]^{T}\right)$ and $\left(1,\left[\begin{array}{ll}\sqrt{3} & 1\end{array}\right]^{T}\right)$, e.g. $\left[\begin{array}{ll}-1 & \sqrt{3}\end{array}\right]\left[\begin{array}{ll}\sqrt{3} w_{1} & w_{1}\end{array}\right]^{T}=0$.

2. In regard to the $\mathbf{w}_{2}$ problem for $\mathbf{x} \in \Sigma_{y}$ Equations 64 and 65 give $\left(\mathbf{I}-\rho_{2}\left(\sigma_{y}\right) \mathbf{R}_{y}\right) \mathbf{w}_{2}=\left[\begin{array}{ll}0 & w_{2_{y}}\end{array}\right]^{T}=\mathbf{0}$ and $\left(\mathbf{I}+\rho_{2}\left(\sigma_{y}\right) \mathbf{R}_{y}\right) \mathbf{t}_{2}=\left[\begin{array}{ll}t_{2} & 0\end{array}\right]^{T}=\mathbf{0}$. Again the $\mathbf{R}_{y}$ eigenpairs $\left(-1,\left[\begin{array}{ll}0 & 1\end{array}\right]^{T}\right)$ and $\left.\left(\begin{array}{lll}1,[ & 0\end{array}\right]^{T}\right)$ are respectively perpendicular to the admissible $\mathbf{w}_{2}=\left[\begin{array}{ll}w_{2} & 0\end{array}\right]^{T}$ and $\mathbf{t}_{2}=\left[\begin{array}{ll}0 & t_{2}\end{array}\right]^{T}$. For $\mathbf{x} \in \Sigma_{y 2}$

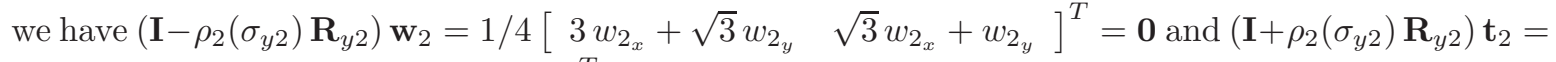
$1 / 4\left[t_{2_{x}}-\sqrt{3} t_{2_{y}}-\sqrt{3} t_{2_{x}}+3 t_{2_{y}}\right]^{T}=\mathbf{0}$. And again the same constraint equation appears twice 
for both $\mathbf{w}_{2}$ and $\mathbf{t}_{2}$ and again the admissible $\mathbf{w}_{2}=\left[\begin{array}{ll}w_{2} & -\sqrt{3} w_{2}\end{array}\right]^{T}$ and $\mathbf{t}_{2}=\left[\begin{array}{ll}\sqrt{3} t_{2} & t_{2}\end{array}\right]^{T}$ are respectively perpendicular to the $\mathbf{R}_{y 2}$ eigenpairs $\left(-1,\left[\begin{array}{ll}\sqrt{3} & 1\end{array}\right]^{T}\right)$ and $\left(1,\left[\begin{array}{ll}-1 & \sqrt{3}\end{array}\right]^{T}\right)$.

3. Now we consider the coupled $\mathbf{w}_{3}^{1}=\left[\begin{array}{ll}\mathbf{w}_{3}^{11^{T}} & \mathbf{w}_{3}^{21^{T}}\end{array}\right]^{T}$ problem. For $\mathbf{x} \in \Sigma_{y}$ we have $\left(\mathbf{I}-\boldsymbol{\rho}_{3}\left(\sigma_{y}\right) \odot\right.$ $\left.\mathbf{R}_{y}\right) \mathbf{w}_{3}^{1}=\left[\begin{array}{llll}w_{3_{x}}^{11} & 0 & 0 & w_{3_{y}}^{21}\end{array}\right]^{T}=\mathbf{0}$ and $\left(\mathbf{I}+\boldsymbol{\rho}_{3}\left(\sigma_{y}\right) \odot \mathbf{R}_{y}\right) \mathbf{t}_{3}^{1}=\left[\begin{array}{llll}0 & t_{3_{y}}^{11} & t_{3_{x}}^{21} & 0\end{array}\right]^{T}=\mathbf{0}$. Again the $\boldsymbol{\rho}_{3}\left(\sigma_{y}\right) \odot \mathbf{R}_{y}$ eigenvectors associated with $\lambda=-1$ pairs, i.e. $\left(-1,\left[\begin{array}{cccc}0 & 0 & 0 & 1\end{array}\right]^{T}\right)$ and $\left(-1,\left[\begin{array}{llll}1 & 0 & 0 & 0\end{array}\right]^{T}\right)$ are perpendicular to the admissible $\mathbf{w}_{3}^{1}=\left[\begin{array}{llll}0 & w_{3}^{11} & w_{3}^{21} & 0\end{array}\right]^{T}$ while the eigenvectors associated with $\lambda=1$ pairs, i.e. $\left(1,\left[\begin{array}{llll}0 & 1 & 0 & 0\end{array}\right]^{T}\right)$ and $\left(1,\left[\begin{array}{llll}0 & 0 & 1 & 0\end{array}\right]^{T}\right)$ are perpendicular to the admissible $\mathbf{t}_{3}^{1}=\left[\begin{array}{llll}t_{3}^{11} & 0 & 0 & t_{3}^{21}\end{array}\right]^{T}$. Note here that the eigenvectors are not unique due to the multiplicity of the $\lambda= \pm 1$ eigenvalues. For $\mathbf{x} \in \Sigma_{y 2}$ we have two independent constraints from

$$
\frac{1}{2}\left(\mathbf{I}-\boldsymbol{\rho}_{3}\left(\sigma_{y 2}\right) \odot \mathbf{R}_{y 2}\right) \mathbf{w}_{3}^{1}=\frac{1}{8}\left[\begin{array}{c}
5 w_{3_{x}}^{11}+\sqrt{3} w_{3_{y}}^{11}+\sqrt{3} w_{3_{x}}^{21}+3 w_{3_{y}}^{21} \\
\sqrt{3} w_{3_{x}}^{11}+3 w_{3_{y}}^{11}+3 w_{3_{x}}^{21}-\sqrt{3} w_{3_{y}}^{21} \\
\sqrt{3} w_{3_{x}}^{11}+3 w_{3_{y}}^{11}+3 w_{3_{x}}^{21}-\sqrt{3} w_{3_{y}}^{21} \\
3 w_{3_{x}}^{11}-\sqrt{3} w_{3_{y}}^{11}-\sqrt{3} w_{3_{x}}^{21}+5 w_{3_{y}}^{21}
\end{array}\right]=\mathbf{0}
$$

and two independent constraints from

$$
\frac{1}{2}\left(\mathbf{I}+\boldsymbol{\rho}_{3}\left(\sigma_{y 2}\right) \odot \mathbf{R}_{y 2}\right) \mathbf{t}_{3}^{1}=\frac{1}{8}\left[\begin{array}{c}
3 t_{3_{x}}^{11}-\sqrt{3} t_{3_{y}}^{11}-\sqrt{3} t_{3_{x}}^{21}-3 t_{3_{y}}^{21} \\
-\sqrt{3} t_{3_{x}}^{11}+5 t_{3_{y}}^{11}-3 t_{3_{x}}^{21}+\sqrt{3} t_{3_{y}}^{21} \\
-\sqrt{3} t_{3_{x}}^{11}-3 t_{3_{y}}^{11}+5 t_{3_{x}}^{21}+\sqrt{3} t_{3_{y}}^{21} \\
-3 t_{3_{x}}^{11}+\sqrt{3} t_{3_{y}}^{11}+\sqrt{3} t_{3_{x}}^{21}+3 t_{3_{y}}^{21}
\end{array}\right]=\mathbf{0} .
$$

Again the $\boldsymbol{\rho}_{3}\left(\sigma_{y 2}\right) \odot \mathbf{R}_{y 2}$ (non-unique) eigenvectors associated with $\lambda=-1$ pairs, i.e. $\left(-1,\left[\begin{array}{cccc}1 & 0 & 0 & 1\end{array}\right]^{T}\right)$ and $\left(-1,[2 / \sqrt{3} \quad 1 \quad 1 \quad 1 \quad 0]^{T}\right)$ are perpendicular to the admissible

$$
\mathbf{w}_{3}^{1}=\left\{\begin{array}{c}
-1 \\
2 / \sqrt{3} \\
0 \\
1
\end{array}\right\} \alpha_{w}+\left\{\begin{array}{c}
0 \\
-1 \\
1 \\
0
\end{array}\right\} \beta_{w}
$$

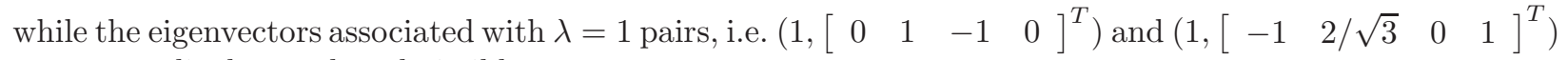
are perpendicular to the admissible

$$
\mathbf{t}_{3}^{1}=\left\{\begin{array}{l}
1 \\
0 \\
0 \\
1
\end{array}\right\} \alpha_{t}+\left\{\begin{array}{c}
2 / \sqrt{3} \\
1 \\
1 \\
0
\end{array}\right\} \beta_{t}
$$

4. Finally, we recall that constraints for the coupled $\mathbf{w}_{3}^{2}=\left[\begin{array}{ll}\mathbf{w}_{3}^{12^{T}} & \mathbf{w}_{3}^{22^{T}}\end{array}\right]^{T}$ problem parrot those of the coupled $\mathbf{w}_{3}^{1}$ problem.

Summarizing, for the $D_{3}$ symmetric domain we solve two uncoupled boundary value problems on $C$. For $\mathbf{w}_{1}$ we satisfy

$$
\begin{array}{rlrl}
\operatorname{div}\left(\mathbb{C}\left[\nabla \mathbf{w}_{1}-\boldsymbol{\chi}_{1}\right]+\mathbf{f}_{1}\right. & =\mathbf{0} & & \text { on } C \\
\mathbf{w}_{1} & =\mathbf{w}_{1}^{p} & \text { on } \partial C^{d} \\
\mathbf{t}_{1} & =\mathbf{t}_{1}^{p} & & \text { on } \partial C^{n} \\
\left\{\begin{array}{c}
w_{1_{x}} \\
t_{2_{y}}
\end{array}\right\} & =\mathbf{0} & & \text { on } \Sigma_{y} \\
\left\{\begin{array}{c}
w_{1_{x}}-\sqrt{3} w_{1_{y}} \\
\sqrt{3} t_{1_{x}}+t_{1_{y}}
\end{array}\right\} & =\mathbf{0} & & \text { on } \Sigma_{y 2}
\end{array}
$$


and for $\mathbf{w}_{2}$ we satisfy

$$
\begin{aligned}
\operatorname{div}\left(\mathbb{C}\left[\nabla \mathbf{w}_{2}-\boldsymbol{\chi}_{2}\right]+\mathbf{f}_{2}\right. & =\mathbf{0} & & \text { on } C \\
\mathbf{w}_{2} & =\mathbf{w}_{2}^{p} & & \text { on } \partial C^{d} \\
\mathbf{t}_{2} & =\mathbf{t}_{2}^{p} & & \text { on } \partial C^{n} \\
\left\{\begin{array}{c}
w_{2_{y}} \\
t_{2_{x}}
\end{array}\right\} & =\mathbf{0} & & \text { on } \Sigma_{y} \\
\left\{\begin{array}{c}
\sqrt{3} w_{2_{x}}+w_{2_{y}} \\
t_{2_{x}}-\sqrt{3} t_{2_{y}}
\end{array}\right\} & =\mathbf{0} & & \text { on } \Sigma_{y 2}
\end{aligned}
$$

We also solve 2 sets of coupled problems for the pairs $\mathbf{w}_{3}^{r}=\left[\mathbf{w}_{3}^{1 r^{T}} \quad \mathbf{w}_{3}^{2 r^{T}}\right]^{T}$ for $r=1,2$ such that

$$
\begin{aligned}
& \operatorname{div}\left(\mathbb{C}\left[\nabla \mathbf{w}_{3}^{l r}-\chi_{3}^{l r}\right]+\mathbf{f}_{3}^{l r}=\mathbf{0} \quad \text { in } C\right. \\
& \mathbf{w}_{3}^{l r}=\mathbf{w}_{3}^{p^{l r}} \quad \text { on } \partial C^{d} \\
& \mathbf{t}_{3}^{l r}=\mathbf{t}_{3}^{p^{l r}} \quad \text { on } \partial C^{n} \\
& \begin{array}{r}
\left\{\begin{array}{c}
w_{3_{x}}^{1 r} \\
w_{3_{y}}^{2 r} \\
t_{3_{y}}^{1 r} \\
t_{3_{x}}^{2 r}
\end{array}\right\}=\mathbf{0} \quad \text { on } \Sigma_{y} \\
\left\{\begin{array}{r}
w_{3_{x}}^{1 r}+w_{3_{y}}^{2 r} \\
2 / \sqrt{3} w_{3_{x}}^{1 r}+w_{3_{y}}^{1 r}+w_{3_{x}}^{2 r} \\
t_{3_{y}}^{1 r}-t_{3_{x}}^{2 r} \\
-t_{3_{x}}^{1 r}+2 / \sqrt{3} w_{3_{y}}^{1 r}+w_{3_{y}}^{2 r}
\end{array}\right\}=\mathbf{0} \quad \text { on } \Sigma_{y 2}
\end{array}
\end{aligned}
$$

where the $\mathbf{w}_{3}^{r}$ and $\mathbf{t}_{3}^{r}$ constraint equations on $\Sigma_{y 2}$ are seen to be equivalent to those of Equations 66 and 67 (which come from the eigenvectors of $\boldsymbol{\rho}_{3}\left(\sigma_{y 2}\right) \odot \mathbf{R}_{y 2}$ ). Again, the Neumann flux conditions on the cut surfaces are necessarily satisfied in the weak formulation whereas the constraints on $\mathbf{w}_{1}, \mathbf{w}_{2}$ and the $\mathbf{w}_{3}^{r}$ are not.

To satisfy the nontrivial constraints we use Lagrange multipliers so that the weak form of Equation 68 , reads: Find $\mathbf{w}_{1} \in \mathcal{H}\left(C, \mathbf{w}_{1}^{p}, 0\right)$ and $\lambda \in \mathcal{L}(C)$ such that

$$
\begin{aligned}
& \int_{C} \nabla \mathbf{v}_{1} \cdot \mathbb{C}\left[\nabla \mathbf{w}_{1}\right] d a+\int_{\Sigma_{y 2}} \beta\left(w_{1_{x}}-\sqrt{3} w_{1_{y}}\right) d l+\int_{\Sigma_{y 2}} \lambda\left(v_{1_{x}}-\sqrt{3} v_{1_{y}}\right) d l= \\
& \int_{C}\left(\nabla \mathbf{v}_{1} \cdot \mathbb{C}\left[\boldsymbol{\chi}_{1}\right]+\mathbf{v}_{1} \cdot \mathbf{f}_{1}\right) d a+\int_{\partial C^{n}} \mathbf{v}_{1} \cdot \mathbf{t}_{1}^{p} d l
\end{aligned}
$$

for all $\mathbf{v}_{1} \in \mathcal{H}(C, \mathbf{0}, 0)$ and $\beta \in \mathcal{L}(C)$ where $\mathcal{H}$ is defined analogous to $\mathcal{H}$ of Equation 36. The weak form of Equation 69 , reads: Find $\mathbf{w}_{2} \in \mathcal{H}\left(C, \mathbf{w}_{2}^{p}, 0\right)$ and $\lambda \in \mathcal{L}(C)$ such that

$$
\begin{aligned}
& \int_{C} \nabla \mathbf{v}_{2} \cdot \mathbb{C}\left[\nabla \mathbf{w}_{2}\right] d a+\int_{\Sigma_{y 2}} \beta\left(\sqrt{3} w_{1_{x}}+w_{1_{y}}\right) d l+\int_{\Sigma_{y 2}} \lambda\left(\sqrt{3} v_{1_{x}}+v_{1_{y}}\right) d l= \\
& \int_{C}\left(\nabla \mathbf{v}_{2} \cdot \mathbb{C}\left[\boldsymbol{\chi}_{2}\right]+\mathbf{v}_{2} \cdot \mathbf{f}_{2}\right) d a+\int_{\partial C^{n}} \mathbf{v}_{2} \cdot \mathbf{t}_{2}^{p} d l
\end{aligned}
$$

for all $\mathbf{v}_{2} \in \mathcal{H}(C, \mathbf{0}, 0)$ and $\beta \in \mathcal{L}(C)$. The weak form for the coupled $\mathbf{w}_{3}^{r}$ reads: Find $\mathbf{w}_{3}^{i r} \in \mathcal{H}\left(C, \mathbf{w}_{3}^{i r^{p}}, 0\right)$ and $\lambda_{i} \in \mathcal{L}(C)$ such that

$$
\begin{aligned}
\int_{C} & \nabla \mathbf{v}_{3}^{1 r} \cdot \mathbb{C}\left[\nabla \mathbf{w}_{3}^{1 r}\right] d a+\int_{C} \nabla \mathbf{v}_{3}^{2 r} \cdot \mathbb{C}\left[\nabla \mathbf{w}_{3}^{2 r}\right] d a+\int_{\Sigma_{y 2}} \beta_{1}\left(w_{3_{x}}^{1 r}+w_{3_{y}}^{2 r}\right) d l+\int_{\Sigma_{y 2}} \beta_{2}\left(2 / \sqrt{3} w_{3_{x}}^{1 r}+w_{3_{y}}^{1 r}+w_{3_{x}}^{2 r}\right) d l+ \\
& \int_{\Sigma_{y 2}} \lambda_{1}\left(v_{3_{x}}^{1 r}+v_{3_{y}}^{2 r}\right) d l+\int_{\Sigma_{y 2}} \lambda_{2}\left(2 / \sqrt{3} v_{3_{x}}^{1 r}+v_{3_{y}}^{1 r}+v_{3_{x}}^{2 r}\right) d l=\int_{C}\left(\nabla \mathbf{v}_{3}^{1 r} \cdot \mathbb{C}\left[\chi_{3}^{1 r}\right]+\mathbf{v}_{3}^{1 r} \cdot \mathbf{f}_{3}^{1 r}\right) d a+ \\
& \int_{C}\left(\nabla \mathbf{v}_{3}^{2 r} \cdot \mathbb{C}\left[\chi_{3}^{2 r}\right]+\mathbf{v}_{3}^{2 r} \cdot \mathbf{f}_{3}^{2 r}\right) d a+\int_{\partial C^{n}} \mathbf{v}_{3}^{1 r} \cdot \mathbf{t}_{3}^{1 r^{p}} d l+\int_{\partial C^{n}} \mathbf{v}_{3}^{2 r} \cdot \mathbf{t}_{3}^{2 r^{p}} d l
\end{aligned}
$$

for all $\mathbf{v}_{3}^{i r} \in \mathcal{H}(C, \mathbf{0}, 0)$ and $\beta_{i} \in \mathcal{L}(C)$. 


\subsection{Homogenization under $D_{3}$ symmetry}

Now we consider the $D_{3}$ symmetric unit cell of Figure 3 for the purpose of homogenization. Using Equation 62 , the unit loads corresponding to $\mathbf{E}_{11}, \mathbf{E}_{21}, \mathbf{E}_{12}$ and $\mathbf{E}_{22}$ require

$$
\begin{aligned}
& \chi_{2}^{(11)}=\chi_{3}^{(11)^{12}}=\chi_{3}^{(11)^{22}}=\mathbf{0}, \chi_{1}^{(11)}=\frac{1}{2}\left(\mathbf{E}_{11}+\mathbf{E}_{22}\right), \chi_{3}^{(11)^{11}}=\frac{1}{2}\left(\mathbf{E}_{11}-\mathbf{E}_{22}\right) \text { and } \chi_{3}^{(11)^{21}}=-\frac{1}{2}\left(\mathbf{E}_{12}+\mathbf{E}_{21}\right) \\
& \chi_{1}^{(12)}=\chi_{3}^{(12)^{11}}=\chi_{3}^{(12)^{21}}=\mathbf{0}, \chi_{2}^{(12)}=\frac{1}{2}\left(\mathbf{E}_{12}-\mathbf{E}_{21}\right), \chi_{3}^{(12)^{12}}=-\chi_{3}^{(11)^{11}} \text { and } \chi_{3}^{(12)^{22}}=-\chi_{3}^{(11)^{21}} \\
& \chi_{1}^{(21)}=\chi_{3}^{(21)^{11}}=\chi_{3}^{(21)^{21}}=\mathbf{0}, \chi_{2}^{(21)}=-\chi_{2}^{(12)}, \chi_{3}^{(21)^{12}}=-\chi_{3}^{(11)^{11}} \text { and } \chi_{3}^{(21)^{22}}=-\chi_{3}^{(11)^{21}} \\
& \chi_{2}^{(22)}=\chi_{3}^{(22)^{12}}=\chi_{3}^{(22)^{22}}=\mathbf{0}, \chi_{1}^{(22)}=\chi_{1}^{(11)}, \chi_{3}^{(22)^{11}}=-\chi_{3}^{(11)^{11}} \text { and } \chi_{3}^{(22)^{21}}=-\chi_{3}^{(11)^{21}}
\end{aligned}
$$

But, due to the symmetry of $\mathbb{C}$, the load $\boldsymbol{\chi}_{2}^{(12)}$ yields zero response since $\mathbb{C}\left[\boldsymbol{\chi}_{2}^{(12)}\right]=\mathbf{0}$; hence we equate $\boldsymbol{\chi}_{2}^{(12)}=$ $\chi_{2}^{(21)}=\mathbf{0}$. Nonetheless, unlike the previous cases we studied, here we need to perform multiple simulations to evaluate the displacement associated with each unit strain, however there is some overlap amongst the problems, e.g. $\chi_{1}^{(11)}=\chi_{1}^{(22)}$. Thus we need only compute $\mathbf{w}_{1}^{(11)}$ and $\mathbf{w}_{3}^{(11)^{1}}=\left[\begin{array}{ll}\mathbf{w}_{3}^{(11)^{11^{T}}} & \mathbf{w}_{3}^{(11)^{21^{T}}}\end{array}\right]^{T}$ as

$$
\begin{aligned}
\mathbf{w}_{3}^{(12)^{12}} & =-\mathbf{w}_{3}^{(11)^{11}} \\
\mathbf{w}_{3}^{(12)^{22}} & =-\mathbf{w}_{3}^{(11)^{21}} \\
\mathbf{w}_{3}^{(21)^{12}} & =-\mathbf{w}_{3}^{(11)^{11}} \\
\mathbf{w}_{3}^{(21)^{22}} & =-\mathbf{w}_{3}^{(11)^{21}} \\
\mathbf{w}_{1}^{(22)} & =\mathbf{w}_{1}^{(11)} \\
\mathbf{w}_{3}^{(22)^{11}} & =-\mathbf{w}_{3}^{(11)^{11}} \\
\mathbf{w}_{3}^{(22)^{21}} & =-\mathbf{w}_{3}^{(11)^{21}} .
\end{aligned}
$$

All the remaining terms are zero.

Now we discuss the periodic boundary conditions as done after Equation 37. For $\mathbf{x} \in \partial C_{1}$, condition $\mathbf{w}\left(\mathbf{R}_{2} \mathbf{x}^{\prime}\right)=\mathbf{w}(\mathbf{x})$ and Equation 59 imply

$$
\begin{aligned}
& \mathbf{w}_{1}(\mathbf{x})=\rho_{1}\left(\sigma_{2}\right) \mathbf{R}_{2} \mathbf{w}_{1}\left(\mathbf{R}_{e} \mathbf{x}\right) \\
& \mathbf{w}_{3}^{r}(\mathbf{x})=\left(\boldsymbol{\rho}_{3}\left(\sigma_{2}\right) \odot \mathbf{R}_{2}\right) \mathbf{w}_{3}^{r}\left(\mathbf{R}_{e} \mathbf{x}\right)
\end{aligned}
$$

which resembles, but is not identical to Equation 64. Expanding the above yields

$$
\begin{aligned}
& \left\{\begin{array}{l}
w_{1_{x}}(\mathbf{x}) \\
w_{1_{y}}(\mathbf{x})
\end{array}\right\}=\frac{1}{2}\left\{\begin{array}{c}
-w_{1_{x}}\left(\mathbf{R}_{e} \mathbf{x}\right)-\sqrt{3} w_{1_{y}}\left(\mathbf{R}_{e} \mathbf{x}\right) \\
\sqrt{3} w_{1_{x}}\left(\mathbf{R}_{e} \mathbf{x}\right)-w_{1_{y}}\left(\mathbf{R}_{e} \mathbf{x}\right)
\end{array}\right\} \\
& \left\{\begin{array}{c}
w_{3_{x}}^{1 r}(\mathbf{x}) \\
w_{3_{y}}^{1 r}(\mathbf{x}) \\
w_{3 x}^{2 r}(\mathbf{x}) \\
w_{3_{y}}^{2 r}(\mathbf{x})
\end{array}\right\}=\frac{1}{4}\left\{\begin{array}{c}
w_{3_{x}}^{1 r}\left(\mathbf{R}_{e} \mathbf{x}\right)+\sqrt{3} w_{3_{y}}^{1 r}\left(\mathbf{R}_{e} \mathbf{x}\right)+\sqrt{3} w_{3_{x}}^{2 r}\left(\mathbf{R}_{e} \mathbf{x}\right)+3 w_{3_{y}}^{2 r}\left(\mathbf{R}_{e} \mathbf{x}\right) \\
-\sqrt{3} w_{3_{x}}^{1 r}\left(\mathbf{R}_{e} \mathbf{x}\right)+w_{3_{y}}^{1 r}\left(\mathbf{R}_{e} \mathbf{x}\right)-3 w_{3_{x}}^{2 r}\left(\mathbf{R}_{e} \mathbf{x}\right)+\sqrt{3} w_{3_{y}}^{2 r}\left(\mathbf{R}_{e} \mathbf{x}\right) \\
-\sqrt{3} w_{3_{x}}^{1 r}\left(\mathbf{R}_{e} \mathbf{x}\right)-3 w_{3_{y}}^{1 r}\left(\mathbf{R}_{e} \mathbf{x}\right)+w_{3_{x}}^{2 r}\left(\mathbf{R}_{e} \mathbf{x}\right)+\sqrt{3} w_{3_{y}}^{2 r}\left(\mathbf{R}_{e} \mathbf{x}\right) \\
3 w_{3_{x}}^{1 r}\left(\mathbf{R}_{e} \mathbf{x}\right)-\sqrt{3} w_{3_{y}}^{1 r}\left(\mathbf{R}_{e} \mathbf{x}\right)-\sqrt{3} w_{3_{x}}^{2 r}\left(\mathbf{R}_{e} \mathbf{x}\right)+w_{3_{y}}^{2 r}\left(\mathbf{R}_{e} \mathbf{x}\right)
\end{array}\right\}
\end{aligned}
$$

for $r=1,2$.

On the other hand, the traction condition $\mathbf{t}\left(\mathbf{R}_{2} \mathbf{x}^{\prime}\right)=-\mathbf{t}(\mathbf{x})$ gives

$$
\begin{aligned}
& \mathbf{t}_{1}(\mathbf{x})=-\rho_{1}\left(\sigma_{2}\right) \mathbf{R}_{2} \mathbf{t}_{1}\left(\mathbf{R}_{e} \mathbf{x}\right) \\
& \mathbf{t}_{3}^{r}(\mathbf{x})=-\left(\boldsymbol{\rho}_{3}\left(\sigma_{2}\right) \odot \mathbf{R}_{2}\right) \mathbf{t}_{3}^{r}\left(\mathbf{R}_{e} \mathbf{x}\right)
\end{aligned}
$$


which expands to

$$
\begin{aligned}
& \left\{\begin{array}{c}
t_{1_{x}}(\mathbf{x}) \\
t_{1_{y}}(\mathbf{x})
\end{array}\right\}=\frac{1}{2}\left\{\begin{array}{c}
t_{1_{x}}\left(\mathbf{R}_{e} \mathbf{x}\right)+\sqrt{3} t_{1_{y}}\left(\mathbf{R}_{e} \mathbf{x}\right) \\
-\sqrt{3} t_{1_{x}}\left(\mathbf{R}_{e} \mathbf{x}\right)+t_{1_{y}}\left(\mathbf{R}_{e} \mathbf{x}\right)
\end{array}\right\} \\
& \left\{\begin{array}{c}
t_{3_{x}}^{1 r}(\mathbf{x}) \\
t_{3_{y}}^{1 r}(\mathbf{x}) \\
t_{3_{x}}^{2 r}(\mathbf{x}) \\
t_{3_{y}}^{2 r}(\mathbf{x})
\end{array}\right\}=\frac{1}{4}\left\{\begin{array}{c}
-t_{3_{x}}^{1 r}\left(\mathbf{R}_{e} \mathbf{x}\right)-\sqrt{3} t_{3_{y}}^{1 r}\left(\mathbf{R}_{e} \mathbf{x}\right)-\sqrt{3} t_{3_{x}}^{2 r}\left(\mathbf{R}_{e} \mathbf{x}\right)-3 t_{3_{y}}^{2 r}\left(\mathbf{R}_{e} \mathbf{x}\right) \\
\sqrt{3} t_{3_{x}}^{1 r}\left(\mathbf{R}_{e} \mathbf{x}\right)-t_{3_{y}}^{1 r}\left(\mathbf{R}_{e} \mathbf{x}\right)+3 t_{3_{x}}^{2 r}\left(\mathbf{R}_{e} \mathbf{x}\right)-\sqrt{3} t_{3_{y}}^{2 r}\left(\mathbf{R}_{e} \mathbf{x}\right) \\
-3 t_{3_{x}}^{1 r}\left(\mathbf{R}_{e} \mathbf{x}\right)+\sqrt{3} t_{3_{y}}^{1 r}\left(\mathbf{R}_{e} \mathbf{x}\right)-t_{3_{x}}^{2 r}\left(\mathbf{R}_{e} \mathbf{x}\right)-\sqrt{3} t_{3_{y}}^{2 r}\left(\mathbf{R}_{e} \mathbf{x}\right)+\sqrt{3} t_{3_{x}}^{2 r}\left(\mathbf{R}_{e} \mathbf{x}\right)-t_{3_{y}}^{2 r}\left(\mathbf{R}_{e} \mathbf{x}\right)
\end{array}\right\}
\end{aligned}
$$

for $r=1,2$.

Similar constraints apply to $\mathbf{y} \in \partial C_{2}$, however, as previously mentioned after Equation 40, no new information is gleaned from these constraints.

Summarizing, the unit load cell problems are defined by Equations $68-70$ with the additional constraints 73 and 74 on $\partial C_{1}$ and the initial strains of Equation 71, cf. Equations $33-34,39$ and 37.

The homogenized material tensor is evaluated with the change of variable theorem, the domain symmetry of $\mathbb{C}$ and Equations 60 and 61, i.e.

$$
\begin{aligned}
& \mathbb{C}_{h}=\frac{1}{\operatorname{vol}(\Omega)} \int_{\Omega} \mathbb{C}(\mathbf{x})\left(\mathbb{\square}+\nabla \mathbf{w}^{(11)}(\mathbf{x}) \otimes \mathbf{E}_{11}+\nabla \mathbf{w}^{(12)}(\mathbf{x}) \otimes \mathbf{E}_{12}+\nabla \mathbf{w}^{(21)}(\mathbf{x}) \otimes \mathbf{E}_{21}+\nabla \mathbf{w}^{(22)}(\mathbf{x}) \otimes \mathbf{E}_{22}\right) d a \\
& =\frac{1}{\operatorname{vol}(\Omega)} \int_{\Omega} \mathbb{C}(\mathbf{x})\left(\square+\left(\nabla \mathbf{w}_{1}^{(11)}(\mathbf{x})+\nabla \mathbf{w}_{3}^{(11)^{11}}(\mathbf{x})\right) \otimes \mathbf{E}_{11}-\nabla \mathbf{w}_{3}^{(11)^{21}}(\mathbf{x}) \otimes \mathbf{E}_{12}-\right. \\
& \left.\nabla \mathbf{w}_{3}^{(11)^{21}}(\mathbf{x}) \otimes \mathbf{E}_{21}+\left(\nabla \mathbf{w}_{1}^{(11)}(\mathbf{x})-\nabla \mathbf{w}_{3}^{(11)^{11}}(\mathbf{x})\right) \otimes \mathbf{E}_{22}\right) d a \\
& =\frac{1}{\operatorname{vol}(\Omega)} \int_{\Omega} \mathbb{C}(\mathbf{x})\left(\mathbb{q}+\nabla \mathbf{w}_{1}^{(11)}(\mathbf{x}) \otimes \mathbf{A}+\nabla \mathbf{w}_{3}^{(11)^{11}}(\mathbf{x}) \otimes \mathbf{B}+\nabla \mathbf{w}_{3}^{(11)^{21}}(\mathbf{x}) \otimes \mathbf{C}\right) d a \\
& =\sum_{g} \frac{1}{\operatorname{vol}(\Omega)} \int_{C}\left(\mathbf{R}_{g} \otimes \mathbf{R}_{g}\right) \mathbb{C}(\mathbf{x})\left(\left(\mathbf{R}_{g} \otimes \mathbf{R}_{g}\right)^{-1}+\rho_{1}\left(\sigma_{g}\right) \nabla \mathbf{w}_{1}^{(11)}(\mathbf{x}) \otimes \mathbf{A}+\right. \\
& \left.\sum_{r=1}^{2} \rho_{3}^{1 r}\left(\sigma_{g}\right) \nabla \mathbf{w}_{3}^{(11)^{r 1}}(\mathbf{x}) \otimes \mathbf{B}+\sum_{r=1}^{2} \rho_{3}^{2 r}\left(\sigma_{g}\right) \nabla \mathbf{w}_{3}^{(11)^{r 1}}(\mathbf{x}) \otimes \mathbf{C}\right) d a
\end{aligned}
$$

where $\mathbf{A}=\mathbf{E}_{11}+\mathbf{E}_{22}=\mathbf{I}, \mathbf{B}=\mathbf{E}_{11}-\mathbf{E}_{22}$ and $\mathbf{C}=-\left(\mathbf{E}_{12}+\mathbf{E}_{21}\right)$.

The finite element implementation follows from that of the scalar case. Referring to Figure 5, nodes on the interior of the surface, i.e. non-corner nodes, are constrained by the $\Sigma_{y}$ equations which are standard, the $\Sigma_{2 y}$ equations which require multi-point constraints and the $\partial C_{1}$ equations which also require multi-point constraints. Nodes $(A, B, C, D)$ belong to multiple constrained surfaces. Arguing as in the scalar case with e.g., $w_{1_{x}}\left(\mathbf{X}^{I}\right)=W_{1_{x}}^{I}$ and $w_{3_{x}}^{i j}\left(\mathbf{X}^{I}\right)=W_{i j_{x}}^{I}$ we only enforce the following constraints for these corner nodes

$$
\begin{array}{rl}
W_{1_{x}}^{A}=W_{1_{y}}^{A}=W_{11_{x}}^{A}=W_{21_{y}}^{A} & =0 \\
W_{1_{x}}^{C}=W_{1_{y}}^{C}=W_{11_{x}}^{C}=W_{11_{y}}^{C}=W_{21_{x}}^{C}=W_{21_{y}}^{C} & 0 \\
W_{1_{x}}^{D} & =0 \\
W_{1_{x}}^{B}-\sqrt{3} W_{1_{y}}^{B} & =0 \\
W_{1_{x}}^{B}-\frac{1}{2}\left(-W_{1_{x}}^{D}-\sqrt{3} W_{1_{y}}^{D}\right) & =0 \\
W_{11_{x}}^{D}=W_{21_{y}}^{D} & =0 \\
2 / \sqrt{3} W_{11_{x}}^{A}+W_{11_{y}}^{A}+W_{21_{x}}^{A} & =0 \\
W_{11_{x}}^{B}+W_{21_{y}}^{B} & =0 \\
2 / \sqrt{3} W_{11_{x}}^{B}+W_{11_{y}}^{B}+W_{21_{x}}^{B} & =0 \\
W_{11_{x}}^{B}-\frac{1}{4}\left(W_{11_{x}}^{D}+\sqrt{3} W_{11_{y}}^{D}+\sqrt{3} W_{21_{x}}^{D}+3 W_{21_{y}}^{D}\right) & =0 \\
W_{11_{y}}^{B}-\frac{1}{4}\left(-\sqrt{3} W_{11_{x}}^{D}+W_{11_{y}}^{D}-3 W_{21_{x}}^{D}+\sqrt{3} W_{21_{y}}^{D}\right) & =0 .
\end{array}
$$




\section{Example}

For our example we use the finite element method to compute the homogenized Young's modulus $E_{h}$ and Poisson's ratio $\nu_{h}$ for the hexagonal $D_{3}$ unit cell of Figure 5. Referring to the figure, the isotropic material in the shaded and unshaded regions have $E=10$ and $\nu=0.3$ and $E=20$ and $\nu=0.3$, respectively. A coarse 8 element mesh of bilinear elements is purposely used to illustrate the deformation fields and specifically how those on the symmetry cell combine to produce those on the full unit cell.

We first perform the analysis on the full unit cell to compute the displacement fields $\mathbf{w}^{(11)}, \mathbf{w}^{(12)}=\mathbf{w}^{(21)}$ and $\mathbf{w}^{(22)}$ corresponding to the unit loads $\mathbf{E}^{(11)}, \mathbf{E}^{(12)}, \mathbf{E}^{(21)}$ and $\mathbf{E}^{(22)}$, cf. Figure 6 . We then perform the analysis on the symmetry cell to compute the displacement fields $\mathbf{w}_{1}^{(11)}, \mathbf{w}_{3}^{(11)^{11}}$ and $\mathbf{w}_{3}^{(11)^{21}}$ corresponding to the unit loads $\boldsymbol{\chi}_{1}^{(11)}=\frac{1}{2}\left(\mathbf{E}_{11}+\mathbf{E}_{22}\right), \boldsymbol{\chi}_{3}^{(11)^{11}}=\frac{1}{2}\left(\mathbf{E}_{11}-\mathbf{E}_{22}\right)$ and $\boldsymbol{\chi}_{3}^{(11)^{21}}=-\frac{1}{2}\left(\mathbf{E}_{12}+\mathbf{E}_{21}\right)$, cf. Figure 7. From Equation 71 we see that $\mathbf{w}^{(11)}=\mathbf{w}_{1}^{(11)}+\mathbf{w}_{3}^{(11)^{11}}, \mathbf{w}^{(12)}=\mathbf{w}^{(21)}=-\mathbf{w}_{3}^{(11)^{21}}$ and $\mathbf{w}^{(22)}=\mathbf{w}_{1}^{(11)}-\mathbf{w}_{3}^{(11)^{11}}$. These relations are visually validated by superimposing the displacement fields illustrated in Figure 7 to obtain those (over the symmetry cell) in Figure 6 . From Figure 7 we can also visually validate constraint Equations 68, 69, 73 and 75. The values of the homogenized Young's modulus and Poisson's ratio, equally computed over either the full unit cell or its symmetry cell, are $E_{h}=14.96$ and $\nu_{h}=0.301$.
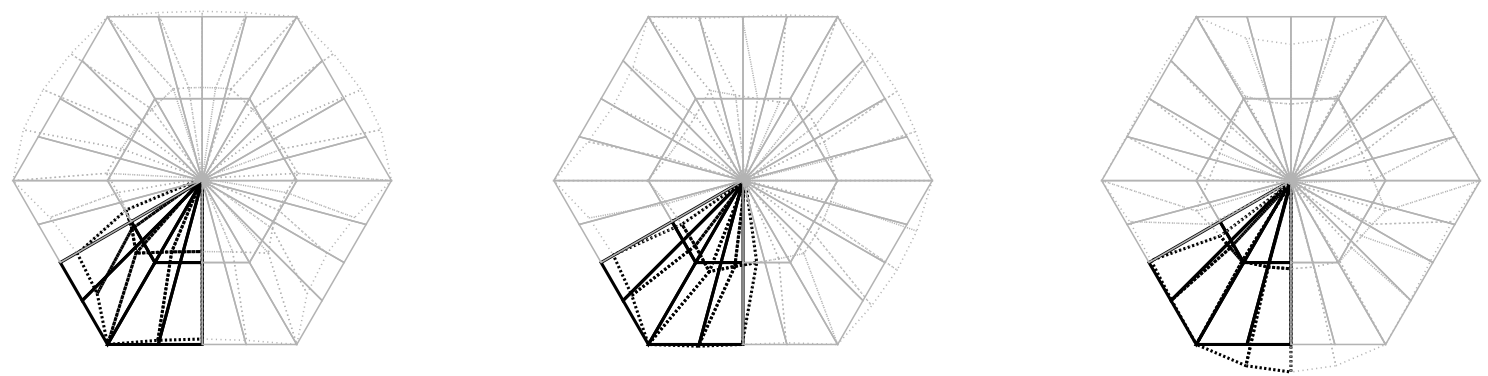

Figure 6: Illustration of displacement fields $\mathbf{w}^{(11)}, \mathbf{w}^{(12)}=\mathbf{w}^{(21)}$ and $\mathbf{w}^{(22)}$.
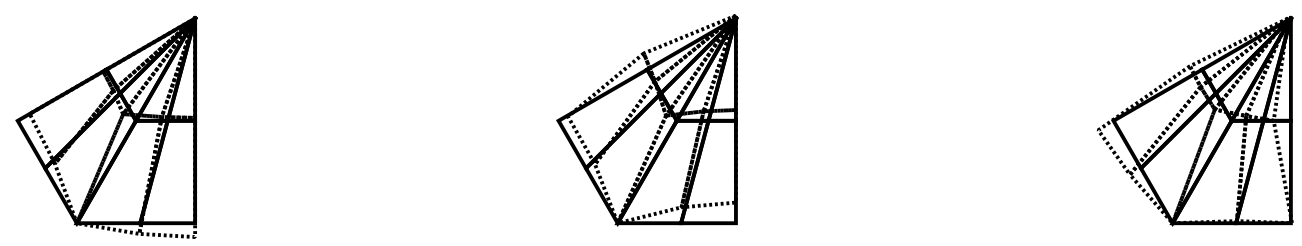

Figure 7: Illustration of displacement fields $\mathbf{w}_{1}^{(11)}, \mathbf{w}_{3}^{(11)^{11}}$ and $\mathbf{w}_{3}^{(11)^{21}}$. 


\section{Conclusions}

When solving a partial differential equation on a domain exhibiting certain symmetries, it is possible to reduce the computational burden by splitting the domain into symmetry cells and by solving several problems on one (small) symmetry cell only, instead of solving a problem on the large domain. For complicated symmetries, one must use group theory in order to obtain the correct boundary constraint conditions on the symmetry cell and to correctly recover the solution on the large domain.

One important aspect is the boundary condition appearing on the new, artificial boundaries resulting from "cutting" the domain into several symmetry cells. On these newly created boundaries, the solution of the PDE is required to satisfy certain conditions which derive from the fact that we do not allow for jumps in either the response or the flux throughout the domain.

In the literature, there are excellent presentations for the application of these procedures to elliptic problems with usual boundary conditions. However, group theory has not played a significant role in the homogenization computations over the unit cells, where one deals with elliptic problems subject to periodicity conditions on the boundary of the cell. It is the purpose of this paper to fill this gap by showing, in a systematic manner, how to take advantage of symmetry properties of the unit cell in order to reduce the original problem to several problems defined on a (smaller) symmetry sector. We investigate domains characterized by abelian and also by nonabelian symmetry groups. In both cases we discuss scalar and vector valued elliptic partial differential equation problems as they relate to, e.g. linear heat conduction and linear elasticity. Special emphasis is put on the dihedral symmetries $D_{2}$ and $D_{3}$, i.e. bodies characterized by 2 and 3 rotational and reflective symmetries. Finally, we apply this theory to evaluate the homogenized properties of a two-phase planar composite over the triangle symmetry sector of a $D_{3}$ symmetric hexagonal unit cell.

These techniques allow for an important reduction in the computational burden. This advantage is more evident in the inverse homogenization framework, where one has to solve the cell primal boundary value problems many times in order to optimize the microstructure layout to maximize the desired homogenized response. The application of the above techniques to inverse homogenization problems is subject to ongoing work. Another direction of future work is how to deal with imperfections in the design which induce a slight violation of the symmetry while the overall structure is still almost symmetric.

\section{Acknowledgements}

The first author acknowledges his support from the by Fundação para a Ciência e a Tecnologia, UID/MAT/04561/2013. The second and third authors acknowledge their support from the U.S. Department of Energy by Lawrence Livermore National Laboratory under Contract DE-AC52-07NA27344 and from DARPAs Materials with Controlled Microstructural Architecture program managed by Dr. Judah Goldwasser.

\section{References}

[1] A. Zingoni. Group-theoretic exploitations of symmetry in computational solid and structural mechanics. International Journal for Numerical Methods in Engineering, 79(3):253-289, 2009.

[2] A. Aimi, L. Bassotti, and M. Diligenti. Groups of congruences and restriction matrices. BIT Numerical Mathematics, 43(4):671-693, 2003.

[3] R.D. Kangwai, S.D. Guest, and S. Pellegrino. Introduction to the analysis of symmetric structures. Computers and Structures, 71(6):671-688, 1999.

[4] T.J. Healey. A group-theoretic approach to computational bifurcation problems with symmetry. Computer Methods in Applied Mechanics and Engineering, 67(3):257-295, 1988.

[5] A. Bossavit. Symmetry, groups, and boundary value problems. a progressive introduction to noncommutative harmonic analysis of partial differential equations in domains with geometrical symmetry. Computer Methods in Applied Mechanics and Engineering, 56(2):167-215, 1986. 
[6] Jacques Lobry and Christian Broche. Exploitation of the geometrical symmetry in the boundary element method with the group representation theory. IEEE Transactions on Magnetics, 30(1):118-124, 1994.

[7] M. Bonnet. Exploiting partial or complete geometrical symmetry in 3d symmetric galerkin indirect bem formulations. International Journal for Numerical Methods in Engineering, 57(8):1053-1083, 2003.

[8] O Sigmund and S Torquato. Design of materials with extreme thermal expansion using a three-phase topology optimization method. JOURNAL OF THE MECHANICS AND PHYSICS OF SOLIDS, 45(6):1037-1067, JUN 1997.

[9] B. Hassani. A direct method to derive the boundary conditions of the homogenization equation for symmetric cells. Communications in Numerical Methods in Engineering, 12(3):185-196, 1996.

[10] D. Lukkassen, A. Meidell, and S. Vigdergauz. On the elastic deformation of symmetric periodic structures. Quarterly Journal of Mechanics and Applied Mathematics, 56(3):441-454, 2003.

[11] J.P. Serre. Linear Representations of Finite Groups. Springer-Verlg, Berlin, 1977.

[12] A. Bensoussan, J.L. Lions, and G. Papanicolaou. Asymptotic Analysis for Periodic Structures. NorthHolland, Amsterdam, 1978.

[13] Seth Watts and Daniel A. Tortorelli. An n-material thresholding method for improving integerness of solutions in topology optimization. International Journal for Numerical Methods in Engineering, to appear, 2016. 